\title{
A NEW CLASS OF RAMSEY-CLASSIFICATION THEOREMS AND THEIR APPLICATIONS IN THE TUKEY THEORY OF ULTRAFILTERS, PART 2
}

\author{
NATASHA DOBRINEN AND STEVO TODORCEVIC
}

\begin{abstract}
Motivated by Tukey classification problems and building on work in Part 1, we develop a new hierarchy of topological Ramsey spaces $\mathcal{R}_{\alpha}$, $\alpha<\omega_{1}$. These spaces form a natural hierarchy of complexity, $\mathcal{R}_{0}$ being the Ellentuck space, and for each $\alpha<\omega_{1}, \mathcal{R}_{\alpha+1}$ coming immediately after $\mathcal{R}_{\alpha}$ in complexity. Associated with each $\mathcal{R}_{\alpha}$ is an ultrafilter $\mathcal{U}_{\alpha}$, which is Ramsey for $\mathcal{R}_{\alpha}$, and in particular, is a rapid p-point satisfying certain partition properties. We prove Ramsey-classification theorems for equivalence relations on fronts on $\mathcal{R}_{\alpha}, 2 \leq \alpha<\omega_{1}$. These form a hierarchy of extensions of the Pudlak-Rödl Theorem canonizing equivalence relations on barriers on the Ellentuck space. We then apply our Ramsey-classification theorems to completely classify all Rudin-Keisler equivalence classes of ultrafilters which are Tukey reducible to $\mathcal{U}_{\alpha}$, for each $2 \leq \alpha<\omega_{1}$ : Every nonprincipal ultrafilter which is Tukey reducible to $\mathcal{U}_{\alpha}$ is isomorphic to a countable iteration of Fubini products of ultrafilters from among a fixed countable collection of rapid p-points. Moreover, we show that the Tukey types of nonprincipal ultrafilters Tukey reducible to $\mathcal{U}_{\alpha}$ form a descending chain of rapid p-points of order type $\alpha+1$.
\end{abstract}

\section{Overview}

This paper builds on and extends work in Part 1 (see [5]) to a large class of new topological Ramsey spaces and their associated ultrafilters. Motivated by a Tukey classification problem and inspired by work of Laflamme in [10] and the second author in [14, we build new topological Ramsey spaces $\mathcal{R}_{\alpha}, 2 \leq \alpha<\omega_{1}$. The space $\mathcal{R}_{0}$ denotes the classical Ellentuck space; the space $\mathcal{R}_{1}$ was built in [5]. The topological Ramsey spaces $\mathcal{R}_{\alpha}, \alpha<\omega_{1}$, form a natural hierarchy in terms of complexity. The space $\mathcal{R}_{1}$ is minimal in complexity above the Ellentuck space, the Ellentuck space being obtained as the projection of $\mathcal{R}_{1}$ via a fixed finite-to-one map. More generally, $\mathcal{R}_{\alpha+1}$ is minimal in complexity over $\mathcal{R}_{\alpha}$ via a fixed finite-to-one map. For limit ordinals $\alpha, \mathcal{R}_{\alpha}$ is formed by diagonalizing in a precise manner over the $\mathcal{R}_{\beta}, \beta<\alpha$. $\mathcal{R}_{\alpha}$ is minimal in complexity over the collection of $\mathcal{R}_{\beta}, \beta<\alpha$, via fixed finite-to-one maps.

Every topological Ramsey space has notions of finite approximations, fronts, and barriers. In [5], we proved that for each $n$, there is a finite collection of canonical

Received by the editors June 13, 2012 and, in revised form, February 12, 2013.

2010 Mathematics Subject Classification. Primary 05D10, 03E02, 06A06, 54D80; Secondary 03E04, 03E05.

The first author was supported by a National Science Foundation - Association for Women in Mathematics Mentoring Grant and a University of Denver Faculty Research Fund Grant.

The second author was supported by grants from NSERC and CNRS. 
equivalence relations for uniform barriers on $\mathcal{R}_{1}$ of rank $n$. In this paper, we prove similar results for all $2 \leq \alpha<\omega_{1}$. In Theorem 4.22, we show that for all $2 \leq \alpha<\omega_{1}$, for any uniform barrier $\mathcal{B}$ on $\mathcal{R}_{\alpha}$ of finite rank and any equivalence relation $\mathrm{E}$ on $\mathcal{B}$, there is an $X \in \mathcal{R}_{\alpha}$ such that $\mathrm{E}$ restricted to the members of $\mathcal{B}$ coming from within $X$ is exactly one of the canonical equivalence relations. For finite $\alpha$, there are finitely many canonical equivalence relations on uniform barriers of finite rank; these are represented by a certain collection of finite trees. Moreover, the numbers of canonical equivalence relations for finite $\alpha$ are given by a recursive function. For infinite $\alpha$, there are infinitely many canonical equivalence relations on uniform barriers of finite rank, represented by tree-like structures. These theorems generalize the Erdős-Rado Theorem [8] for uniform barriers of finite rank on the Ellentuck space, namely, those of the form $[\mathbb{N}]^{n}$.

In the main theorem of this paper, Theorem 4.12, we prove new Ramsey-classification theorems for all barriers (and moreover all fronts) on the topological Ramsey spaces $\mathcal{R}_{\alpha}, 2 \leq \alpha<\omega_{1}$. We prove that for any barrier $\mathcal{B}$ on $\mathcal{R}_{\alpha}$ and any equivalence relation on $\mathcal{B}$, there is an inner Sperner map which canonizes the equivalence relation. This generalizes our analogous theorem for $\mathcal{R}_{1}$ in [5], which in turn generalized the Pudlak-Rödl Theorem [13] for barriers on the Ellentuck space. These classification theorems were motivated by the following.

Recently the second author (see Theorem 24 in [14]) has made a connection between the Ramsey-classification theory (also known as the canonical Ramsey theory) and the Tukey classification theory of ultrafilters on $\omega$. More precisely, he showed that selective ultrafilters realize minimal Tukey types in the class of all ultrafilters on $\omega$ by applying the Pudlak-Rödl Ramsey classification result to a given cofinal map from a selective ultrafilter into any other ultrafilter on $\omega$, a map which, on the basis of our previous paper [4], he could assume to be continuous. Recall that the notion of a selective ultrafilter is closely tied to the Ellentuck space on the family of all infinite subsets of $\omega$, or rather the one-dimensional version of the pigeonhole principle on which the Ellentuck space is based, the principle stating that an arbitrary $f: \omega \rightarrow \omega$ is either constant or is one-to-one on an infinite subset of $\omega$. Thus an ultrafilter $\mathcal{U}$ on $\omega$ is selective if for every map $f: \omega \rightarrow \omega$ there is an $X \in \mathcal{U}$ such that $f$ is either constant or one-to-one on $X$. Since essentially any other topological Ramsey space has it own notion of a selective ultrafilter living on the set of its 1-approximations (see [11]), the argument for Theorem 24 in [14] is so general that it will give analogous Tukey classification results for all ultrafilters of this sort provided, of course, that we have the analogues of the Pudlak-Rödl Ramsey-classification result for the corresponding topological Ramsey spaces.

In [10, Laflamme forced ultrafilters, $\mathcal{U}_{\alpha}, 1 \leq \alpha<\omega_{1}$, which are rapid p-points satisfying certain partition properties and which have complete combinatorics over the Solovay model. Laflamme showed that, for each $1 \leq \alpha<\omega_{1}$, the Rudin-Keisler equivalence classes of all ultrafilters Rudin-Keisler below $\mathcal{U}_{\alpha}$ form a descending chain of order type $\alpha+1$. This employed a result of Blass in 2] which states that each weakly Ramsey ultrafilter has exactly one Rudin-Keisler type below it, namely the isomorphism class of a selective ultrafilter. We review these results now.

Recall from [10] that an ultrafilter $\mathcal{U}$ is said to satisfy the $(n, k)$ Ramsey partition property (or $\mathrm{RP}^{n}(k)$ ) if for all functions $f:[\omega]^{k} \rightarrow n^{k-1}+1$ and all partitions $\left\langle A_{m}: m \in \omega\right\rangle$ of $\omega$ with each $A_{m} \notin \mathcal{U}$, there is a set $X \in \mathcal{U}$ such that $\left|X \cap A_{m}\right|<\omega$ for each $m<\omega$, and $\left|f^{\prime \prime}\left[A_{m} \cap X\right]^{2}\right| \leq n^{k-1}$ for each $m<\omega$. 
Theorem 1.1 (Laflamme [10]). For each $1 \leq \alpha<\omega_{1}$, there is an ultrafilter $\mathcal{U}_{\alpha}$, forced by a $\sigma$-complete forcing $\mathbb{P}_{\alpha}$, with the following properties:

(1) For each $1 \leq \alpha<\omega, \mathcal{U}_{\alpha}$ is a rapid p-point which has complete combinatorics.

(2) For each $1 \leq n<\omega, \mathcal{U}_{n}$ satisfies the $(n, k)$ Ramsey partition property for all $k \geq 1$. For $\omega \leq \alpha<\omega_{1}, \mathcal{U}_{\alpha}$ satisfies analogous Ramsey partition properties.

(3) The isomorphism types of all nonprincipal ultrafiters Rudin-Keisler reducible to $\mathcal{U}_{\alpha}$ forms a strictly decreasing chain of order type $\alpha+1$.

(4) $\mathcal{U}_{1}$ is weakly Ramsey but not Ramsey.

One line of motivation for the work in this paper was to find the structure of the Tukey types of all nonprincipal ultrafilters Tukey reducible to $\mathcal{U}_{\alpha}$, for all $1 \leq \alpha<\omega_{1}$. In Part 1 , we accomplished this for $\mathcal{U}_{1}$, proving that there is exactly one Tukey type of nonprincipal ultrafilter Tukey strictly reducible to $\mathcal{U}_{1}$, namely that of the projection of $\mathcal{U}_{1}$ to a Ramsey ultrafilter. Here, we show in Theorem 5.13 that, in fact, the Tukey types of nonprincipal ultrafilters below that of $\mathcal{U}_{\alpha}$ form a descending chain of order type $\alpha+1$. Thus, the structure of the Tukey types below $\mathcal{U}_{\alpha}$ is the same as the structure of the Rudin-Keisler equivalence classes below $\mathcal{U}_{\alpha}$.

The stronger motivations were to find new canonization theorems for equivalence relations on fronts on $\mathcal{R}_{\alpha}, 2 \leq \alpha<\omega_{1}$, and to apply them to completely classify all Rudin-Keisler types within the Tukey types of the ultrafilters $\mathcal{U}_{\alpha}$, obtaining results finer than Theorem 5.13. Each of the spaces $\mathcal{R}_{\alpha}$ is constructed to give rise to an ultrafilter which is isomorphic to Laflamme's $\mathcal{U}_{\alpha}$. We point out that the fact that each $\mathcal{U}_{\alpha}$ arises from a topological Ramsey space gives a precise explanation of the notion of 'complete combinatorics' of Blass and Laflamme. (See [5] for a detailed discussion.) The canonization Theorems 4.22 and 4.12 generalize results in [5], which in turn had generalized the Erdős-Rado Theorem for barriers of the form $[\mathbb{N}]^{n}$ and the Pudlak-Rödl Theorem for general barriers on the Ellentuck space, respectively. Applying Theorem 4.12, we completely classify all Rudin-Keisler classes of ultrafilters which are Tukey reducible to $\mathcal{U}_{\alpha}$ in Theorem 5.11] These turn out to be exactly the countable iterations of Fubini products of rapid p-points obtained as projections of $\mathcal{U}_{\alpha}$ via canonical equivalence relations. This extends Theorem 5.10 in [5], which classified the Rudin-Keisler classes of ultrafilters Tukey reducible to $\mathcal{U}_{1}$. We remark that the fact that each $\mathcal{R}_{\alpha}$ is a topological Ramsey space is essential to the proof of Theorem [5.11, and that forcing alone is not sufficient to obtain our results.

The main new contributions in this work, as opposed to straightforward generalizations of the work in [5], are the following. First, the cases when $\alpha$ is infinite necessitate a new way of constructing the spaces $\mathcal{R}_{\alpha}$. The base trees $\mathbb{T}_{\alpha}$ for the spaces $\mathcal{R}_{\alpha}$ must be well-founded in order to generate topological Ramsey spaces. However, the true structures are best captured by tree-like objects $\mathbb{S}_{\alpha}$ which are neither truly trees nor well-founded. These new auxiliary structures $\mathbb{S}_{\alpha}$ are also needed to make the canonical equivalence relations precise. Second, we provide a general induction scheme by which we prove the Ramsey-classification theorems for $\mathcal{R}_{\alpha}$, for all $\alpha<\omega_{1}$. The proof that $\mathcal{R}_{\alpha}$ is a topological Ramsey space uses the Ramsey-classification theorems for all $\mathcal{R}_{\beta}, \beta<\alpha$. Third, new sorts of structures appear within the collection of all Rudin-Keisler equivalence classes lying within the Tukey type of $\mathcal{U}_{\alpha}$, for $\alpha \geq 2$. The isomorphism types of the p-points in the Tukey type of $\mathcal{U}_{\alpha}$ are exactly computed from the canonical equivalence relations on 
the $\mathcal{R}_{\alpha}(n)$. Every ultrafilter Tukey equivalent to $\mathcal{U}_{\alpha}$ is isomorphic to some iterated Fubini product of such p-points. Thus, many chains, antichains, and lattice-like structures embed into the isomorphism types within $\left[\mathcal{U}_{\alpha}\right]_{T}$. We discuss this in detail at the end of Section 5. Taken together, these constitute the first known transfinite collection of topological Ramsey spaces and associated ultrafilters which, though very different from Ramsey, behave quite similarly to Ramsey ultrafilters.

The space $\mathcal{R}_{1}$ and the main results in [5] form the starting point for the work in this paper. The spaces $\mathcal{R}_{\alpha}, \alpha<\omega_{1}$, are constructed in Section 2 , based on infinitely wide, well-founded trees $\mathbb{T}_{\alpha}$. The fact that $\alpha$ may now be infinite necessitates a new construction of the base trees $\mathbb{T}_{\alpha}$ for the spaces $\mathcal{R}_{\alpha}$, using auxiliary structures $\mathbb{S}_{\alpha}$ to preserve information about how the trees were built. A new analysis of the canonical equivalence relations is also necessary in this context. See Section 2 for more discussion of these issues. By an induction on $2 \leq \alpha<\omega_{1}$ cycling through Sections 3 and 4 each $\mathcal{R}_{\alpha}$ is proved to be a topological Ramsey space (Theorem 3.14), and the main theorem of this paper, the Ramsey-classification Theorem 4.12 for $\mathcal{R}_{\alpha}$, is proved for each $2 \leq \alpha<\omega_{1}$. The classification of the isomorphism types within the Tukey types of the $\mathcal{U}_{\alpha}$ is proved in Section 5. We then provide examples of structures which embed into the isomorphism types within each Tukey type of $\mathcal{U}_{\alpha}$. These are coded by the substructures of the $\mathbb{S}_{\alpha}$ along with iterated Fubini products. We proceed to the main work of this paper, referring the reader to [5] for further background and definitions.

The authors would like to thank Jordi Lopez-Abad for his generous help in producing graphics of the trees and structures in Section 2.

\section{Construction of trees $\mathbb{T}_{\alpha}$ And the spaces $\left(\mathcal{R}_{\alpha}, \leq_{\alpha}, r^{\alpha}\right)$, FOR $\alpha<\omega_{1}$}

By recursion on $\alpha<\omega_{1}$, we construct trees $\mathbb{T}_{\alpha}$, related auxiliary structures $\mathbb{S}_{\alpha}$, and maps $\tau_{\beta, \alpha}, \sigma_{\beta, \alpha}, \psi_{\alpha}$, for $\beta<\alpha$. After completing this recursive definition, we then define the spaces $\mathcal{R}_{\alpha}$. These spaces are modified versions of dense subsets of the forcings $\mathbb{P}_{\alpha}$ of Laflamme in [10]. The main difference is that we pair down his forcings and use trees and related structures instead of finite sets in such a way as will produce topological Ramsey spaces.

The purpose of the $\mathbb{S}_{\alpha}$ is several-fold. They aid in the precision of the definitions of members of $\mathcal{R}_{\alpha}$ while having the members of $\mathcal{R}_{\alpha}$ be well-founded trees (hence countable objects). They also provide a simple way of understanding the canonical equivalence relations in terms of downward closed subsets of the $\mathbb{S}_{\alpha}(n)$. This in turn makes clear the structures of the Rudin-Keisler types and Tukey types of all ultrafilters Rudin-Keisler or Tukey reducible to $\mathcal{U}_{\alpha}$. For $\alpha \geq \omega, \mathbb{S}_{\alpha}$ will not truly be a tree, but will have a tree-like structure under the ordering of $\subset$. Downward closed subsets of $\mathbb{S}_{\alpha}$ will be chains which are well-ordered by the reverse ordering $\supset$ on $\mathbb{S}_{\alpha}$. This may seem a bit strange at first, but the $\mathbb{S}_{\alpha}$ 's are in fact the correct structures, completely and precisely capturing the structure of the spaces $\mathcal{R}_{\alpha}$.

The maps $\psi_{\alpha}: \mathbb{S}_{\alpha} \rightarrow \mathbb{T}_{\alpha}$ are to be thought of as projection maps, projecting the structure of $\mathbb{S}_{\alpha}$ onto the tree $\mathbb{T}_{\alpha}$. For $\alpha<\omega \cdot \omega, \tau_{\beta, \alpha}$ will be the projection map from $\mathbb{T}_{\alpha}$ to $\mathbb{T}_{\beta}$ and $\sigma_{\beta, \alpha}$ will be the projection map from $\mathbb{S}_{\alpha}$ to $\mathbb{S}_{\beta}$. For $\alpha \geq \omega \cdot \omega$, this will almost be the case: Properties $(\dagger)$ and $(\ddagger)$ below will be preserved.

Let $\mathcal{R}_{0}$ denote the Ellentuck space. For the recursive construction of $\mathcal{R}_{1}$ from $\mathcal{R}_{0}$, it is useful to represent the Ellentuck space as a space of trees as follows. Let $\mathbb{T}_{0}$ denote the tree ${ }^{\leq 1} \mathbb{N}$ of height 1 and infinite width. The members of $\mathcal{R}_{0}$ are all 
infinite subtrees of $\mathbb{T}_{0}$. For $X, Y \in \mathcal{R}_{0}, Y \leq_{0} X$ iff $Y \subseteq X$. Let $\mathbb{S}_{0}=\mathbb{T}_{0}$ and $\psi_{0}$ be the identity map from $\mathbb{S}_{0}$ to $\mathbb{T}_{0}$.

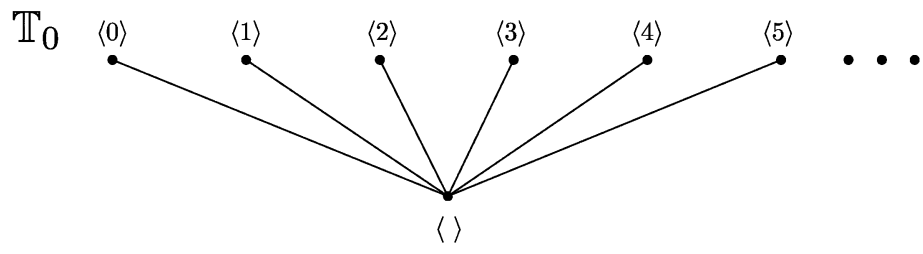

Figure 1. The tree $\mathbb{T}_{0}$

In order to accommodate the recursive definitions of the trees $\mathbb{T}_{\alpha}, 1 \leq \alpha<$ $\omega_{1}$, we very slightly modify the definition of $\mathbb{T}_{1}$ from [5] by changing $\mathbb{T}_{1}(0)$ from $\{\langle\rangle,\langle 0\rangle,\langle 0,0\rangle\}$ to $\{\langle\rangle,\langle 0\rangle\}$. The structure $\mathbb{S}_{1}$ here is exactly the structure $\mathbb{T}_{1}$ from [5]. The reader familiar with that paper will immediately see that this re-definition does not change any of the results in there. In fact, we could use the same definition of $\mathbb{T}_{1}$ here as in [5] and just define all trees $\mathbb{T}_{n}$ below to be exactly $\mathbb{S}_{n}$, for all $n<\omega$. The shortcoming of that approach is that it will not lead to a recursive definition for $\mathbb{T}_{\alpha}, \omega \leq \alpha<\omega_{1}$.

Definition 2.1 $\left(\mathbb{T}_{1}, \tau_{0,1}, \mathbb{S}_{1}, \sigma_{0,1}, \psi_{1}\right)$. Let $l_{0}^{0}=0, l_{1}^{0}=1, l_{2}^{0}=3$, and generally, $l_{n+1}^{0}=l_{n}^{0}+n+1$, for $n \geq 2$. Define

$$
\mathbb{T}_{1}(0)=\{\langle\rangle,\langle 0\rangle\}
$$

For $0<n<\omega$, let

$$
\mathbb{T}_{1}(n)=\left\{\langle\rangle,\langle n\rangle,\langle n, i\rangle: l_{n}^{0} \leq i<l_{n+1}^{0}\right\}
$$

Let

$$
\mathbb{T}_{1}=\bigcup_{n<\omega} \mathbb{T}_{1}(n)
$$

Note that $\mathbb{T}_{1}$ is a tree, ordered by end-extension, which is a substructure of $\leq 2 \mathbb{N}$.

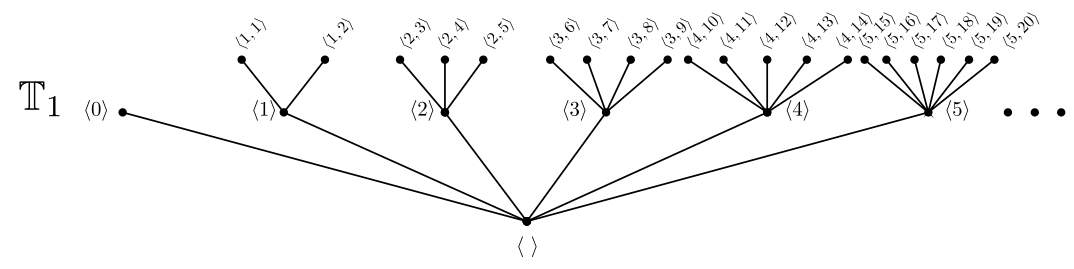

Figure 2. The tree $\mathbb{T}_{1}$

Define $\tau_{0,1}: \mathbb{T}_{1} \rightarrow \mathbb{T}_{0}$, the projection of $\mathbb{T}_{1}$ to $\mathbb{T}_{0}$, by

$$
\begin{aligned}
\tau_{0,1}(\langle 0\rangle) & =\langle 0\rangle ; \\
\tau_{0,1}(t) & =\langle t(1)\rangle, \text { if }|t|=2 ; \\
\tau_{0,1}(t) & =\langle\rangle, \text { if } t=\langle n\rangle \text { and } n \neq 0 \text { or } t=\langle\rangle .
\end{aligned}
$$

Define the auxiliary structure $\mathbb{S}_{1}$ as follows. For each $n<\omega$, let $\mathbb{S}_{1}(n)$ be the collection of functions $f$ with domain $\{0,1\},\{1\}$, or $\emptyset$ such that if $0 \in \operatorname{dom}(f)$, 
then $l_{n}^{0} \leq f(0)<l_{n+1}^{0}$, and if $1 \in \operatorname{dom}(f)$, then $f(1)=n$. Let $\mathbb{S}_{1}=\bigcup_{n<\omega} \mathbb{S}_{1}(n)$. Note that $\mathbb{S}_{1}$ forms a tree structure under extension. For example, $\{(1,1)\} \subset$ $\{(0,1),(1,1)\}$ in the extension ordering on $\mathbb{S}_{1}$.

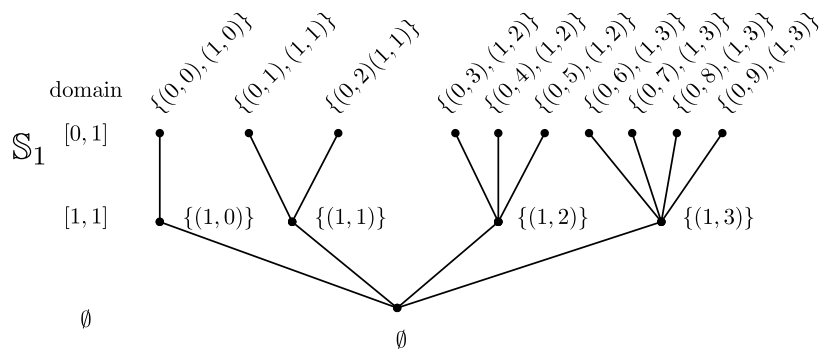

FIgURE 3. The tree $\mathbb{S}_{1}$

Define $\sigma_{0,1}: \mathbb{S}_{1} \rightarrow \mathbb{S}_{0}$, the projection of $\mathbb{S}_{1}$ to $\mathbb{S}_{0}$ by $\sigma_{0,1}(s)=s \uparrow\{0\}$, for each $s \in \mathbb{S}_{1}$. For each $n<\omega$, there is a natural projection map $\psi_{1}: \mathbb{S}_{1} \rightarrow \mathbb{T}_{1}$ such that for each $n<\omega, \psi_{1}^{\prime \prime} \mathbb{S}_{1}(n)=\mathbb{T}_{1}(n)$. This map is defined by

$$
\begin{aligned}
\psi_{1}(\{(0,0),(1,0)\}) & =\psi_{1}(\{(1,0)\})=\langle 0\rangle ; \\
\psi_{1}(\{(0, i),(1, n)\}) & =\langle n, i\rangle, \text { for } n \geq 1 ; \\
\psi_{1}(\{(1, n)\}) & =\langle n\rangle, \text { for } n \geq 1 ; \\
\psi_{1}(\{\emptyset\}) & =\langle\rangle .
\end{aligned}
$$

Remark 2.2. $\mathbb{S}_{1}$ has a tree structure under the ordering $\subset$, but with the domain of the sequences reversed in order. This is done so that it will be clear exactly how $\mathbb{S}_{\alpha}$ is built from $\mathbb{S}_{\beta}$, for $\beta<\alpha$, and also to aid in understanding the Rudin-Keisler ordering on the ultrafilters $\mathcal{U}_{\alpha}$ Ramsey for the spaces $\mathcal{R}_{\alpha}$.

In preparation for the recursive construction, assume that we have fixed, for each limit ordinal $\alpha<\omega_{1}$, a strictly increasing cofinal function $c_{\alpha}: \omega \rightarrow \alpha$. For $\alpha=\omega \cdot(n+1)$ for $n<\omega$, we may take $c_{\alpha}: \omega \rightarrow \alpha$ to be given by $c_{\alpha}(m)=\omega \cdot n+m$. Though not necessary, this does make the spaces $\mathbb{T}_{\alpha}, \alpha<\omega \cdot \omega$ very clear.

Given that $\mathbb{T}_{\beta}$ and $\mathbb{S}_{\beta}$ have been defined, we define the maps $\sigma_{\gamma, \beta}$ and $\tau_{\gamma, \beta}$ for all $\gamma<\beta$ as follows. Define $\sigma_{\gamma, \beta}$ on $\mathbb{S}_{\beta}$ by $\sigma_{\gamma, \beta}(s)=s \uparrow(\gamma+1)$, for each $s \in \mathbb{S}_{\beta}$. Hence, if $\operatorname{dom}(s)=[\zeta, \beta]$ with $\zeta \leq \gamma$, then $\sigma_{\gamma, \beta}(s)=s \uparrow[\zeta, \gamma]$; and if $\gamma<\zeta \leq \beta$, then $\sigma_{\gamma, \beta}(s)=\emptyset$. Note that for each $t \in \mathbb{T}_{\beta}, \psi_{\gamma} \circ \sigma_{\gamma, \beta} \circ \psi_{\beta}^{-1}(t)$ is a singleton. (The singleton can be the set containing the empty sequence.) Define $\tau_{\gamma, \beta}(t)$ to be the member of $\psi_{\gamma} \circ \sigma_{\gamma, \beta} \circ \psi_{\beta}^{-1}(t)$.

By our choices of the functions $c_{\alpha}$ for $\alpha<\omega \cdot \omega$, it follows that for all $\gamma<\beta<\omega \cdot \omega$, $\sigma_{\gamma, \beta}: \mathbb{S}_{\beta} \rightarrow \mathbb{S}_{\gamma}$ and $\tau_{\gamma, \beta}: \mathbb{T}_{\beta} \rightarrow \mathbb{T}_{\gamma}$. For $\gamma \geq \omega \cdot \omega$, this will not necessarily be the case. However, the following properties $(\dagger)$ and $(\ddagger)$ hold for $\beta=1$, and we will preserve them for all $\beta<\omega_{1}$. For $m<\omega$, we shall let $\mathbb{S}_{\beta}([m, \omega))$ denote $\bigcup\left\{\mathbb{S}_{\beta}(n): m \leq n<\omega\right\}$.

$(\dagger)$ For each $\gamma \leq \beta$, there is a $k<\omega$ such that for each $l \geq k$, there is an $m<\omega$ such that $\mathbb{S}_{\gamma}(l) \subseteq \sigma_{\gamma, \beta}\left(\mathbb{S}_{\beta}(m)\right)$.

In particular, there are $k, m<\omega$ such that $\sigma_{\gamma, \beta}\left(\mathbb{S}_{\beta}([k, \omega))\right)=\mathbb{S}_{\gamma}([m, \omega))$.

$(\ddagger)$ For each $\gamma \leq \beta$, there is a $k<\omega$ such that for each $l \geq k$, there is an $m<\omega$ such that $\mathbb{T}_{\gamma}(l) \subseteq \tau_{\gamma, \beta}\left(\mathbb{T}_{\beta}(m)\right)$. 
Induction Assumption for $1<\alpha<\omega_{1}$. Let $1<\alpha<\omega_{1}$ and suppose that for all $\beta<\alpha$ we have defined $\mathbb{T}_{\beta}, \mathbb{S}_{\beta}, \psi_{\beta}$, and for all $\gamma<\beta<\alpha$, we have defined $\sigma_{\gamma, \beta}, \tau_{\gamma, \beta}$ so that $(\dagger)$ and $(\ddagger)$ hold.

There are two cases for the induction step: either $\alpha$ is a successor ordinal or else $\alpha$ is a limit ordinal.

Definition $2.3\left(\mathbb{T}_{\alpha}, \mathbb{S}_{\alpha}, \psi_{\alpha}, \alpha\right.$ a successor ordinal). Suppose that $\alpha=\delta+k+1$, where $\delta$ is either 0 or a countable limit ordinal and $k<\omega$. For $n \leq k+1$, define $l_{n}^{\delta+k}=n$, and for $n \geq k+1$, define $l_{n+1}^{k}=l_{n}^{k}+(n+1)-k$. For each $n \leq k$, let

$$
\mathbb{T}_{\alpha}(n)=\mathbb{T}_{\delta+k}(n) .
$$

For each $n>k$, let

$$
\mathbb{T}_{\alpha}(n)=\{\langle\rangle\} \cup\left\{\langle n\rangle \frown t: t \in \bigcup\left\{\mathbb{T}_{\delta+k}(i): l_{n}^{\delta+k} \leq i<l_{n+1}^{\delta+k}\right\}\right\} .
$$

Let

$$
\mathbb{T}_{\alpha}=\bigcup\left\{\mathbb{T}_{\alpha}(n): n<\omega\right\} .
$$

For each $n<\omega$, define $\mathbb{S}_{\alpha}(n)$ to consist of the empty set along with all functions $f \uparrow[\beta, \alpha], \beta \leq \alpha$, where $f=g \cup\{(\alpha, n)\}$ for some $g \in \bigcup\left\{\mathbb{S}_{\delta+k}(l): l_{n}^{\delta+k} \leq l<l_{n+1}^{\delta+k}\right\}$ with $\operatorname{dom}(g)=[0, \delta+k]$. Let $\mathbb{S}_{\alpha}=\bigcup_{n<\omega} \mathbb{S}_{\alpha}(n)$.

There is a natural projection map $\psi_{\alpha}: \mathbb{S}_{\alpha} \rightarrow \mathbb{T}_{\alpha}$ such that for each $n<\omega$, $\psi_{\alpha}^{\prime \prime} \mathbb{S}_{\alpha}(n)=\mathbb{T}_{\alpha}(n)$, defined as follows. Let $s \in \mathbb{S}_{\alpha}(n)$. If $\operatorname{dom}(s)=\emptyset$, then let $\psi_{\alpha}(s)=\langle\rangle$. If $\operatorname{dom}(s)=[\alpha, \alpha]$, then let $\psi_{\alpha}(s)=\langle n\rangle$. Now suppose $\operatorname{dom}(s)=[\zeta, \alpha]$ where $\zeta<\alpha$. If $n \leq k$, then let $\psi_{\alpha}(s)=\psi_{\delta+k}(s \uparrow[\zeta, \delta+k])$. If $n>k$, then let $\psi_{\alpha}(s)=\langle n\rangle \frown \psi_{\delta+k}(s \uparrow[\zeta, \delta+k])$.

Definition $2.4\left(\mathbb{T}_{\alpha}, \mathbb{S}_{\alpha}, \psi_{\alpha}, \alpha\right.$ a limit ordinal). For $n=0$, letting $\gamma=c_{\alpha}(0)<$ $\beta=c_{\alpha}(1)<\alpha$, by $(\ddagger)$ there is a $k_{0}$ such that for each $k \geq k_{0}$, there is an $m$ such that $\mathbb{T}_{c_{\alpha}(0)}(k) \subseteq \tau_{c_{\alpha}(0), c_{\alpha}(1)}\left(\mathbb{T}_{c_{\alpha}(1)}(m)\right)$. Choose the least such $k_{0}$ and fix $m_{0}$ such that $\mathbb{T}_{c_{\alpha}(0)}\left(k_{0}\right) \subseteq \tau_{c_{\alpha}(0), c_{\alpha}(1)}\left(\mathbb{T}_{c_{\alpha}(1)}\left(m_{0}\right)\right)$ and let $l_{0}$ be the largest integer such that $\mathbb{T}_{c_{\alpha}(0)}\left(l_{0}\right) \subseteq \mathbb{T}_{c_{\alpha}(1)}\left(m_{0}\right)$. For each $i \leq l_{0}$, let

$$
\mathbb{T}_{\alpha}(i)=\mathbb{T}_{c_{\alpha}(0)}(i) \text {. }
$$

Define $p_{-1}=0$ and $p_{0}=l_{0}$.

Assume we have defined $\mathbb{T}_{\alpha}(i)$ for all $i \leq p_{n}$ such that

(1) For each $p_{n-1}<i \leq p_{n}, \mathbb{T}_{\alpha}(i)=\mathbb{T}_{c_{\alpha}(n)}(m)$ for some $m$;

and for some $l_{n}, m_{n}$ :

(2) $\mathbb{T}_{\alpha}\left(p_{n}\right)=\mathbb{T}_{c_{\alpha}(n)}\left(l_{n}\right)$;

(3) $\mathbb{T}_{c_{\alpha}(n)}\left(l_{n}\right) \subseteq \mathbb{T}_{c_{\alpha}(n+1)}\left(m_{n}\right)$, and $l_{n}$ is the largest such;

(4) for all $q \geq \bar{l}_{n}$, there is an $m$ such that $\mathbb{T}_{c_{\alpha}(n)}(q) \subseteq \mathbb{T}_{c_{\alpha}(n+1)}(m)$.

Use $(\ddagger)$ to find a $k_{n+1}$ such that for each $q \geq k_{n+1}$, there is an $m$ such that $\mathbb{T}_{c_{\alpha}(n+1)}(q) \subseteq \mathbb{T}_{c_{\alpha}(n+2)}(m)$. Choose the least such $k_{n+1} \geq m_{n}$ and fix $m_{n+1}$ such that $\mathbb{T}_{c_{\alpha}(n+1)}\left(k_{n+1}\right) \subseteq \mathbb{T}_{c_{\alpha}(n+2)}\left(m_{n+1}\right)$ and let $l_{n+1}$ be the largest integer such that $\mathbb{T}_{c_{\alpha}(n+1)}\left(l_{n+1}\right) \subseteq \mathbb{T}_{c_{\alpha}(n+2)}\left(m_{n+1}\right)$. Put

$$
\mathbb{T}_{\alpha}(i)=\mathbb{T}_{c_{\alpha}(n+1)}\left(m_{n}+i-p_{n}\right),
$$

for $i=p_{n}+1, \ldots, p_{n}+l_{n+1}-m_{n}:=p_{n+1}$. Let

$$
\mathbb{T}_{\alpha}=\bigcup\left\{\mathbb{T}_{\alpha}(j): j<\omega\right\} .
$$


Note that $(\ddagger)$ is preserved up to and including $\alpha$ by this construction.

Define $\mathbb{S}_{\alpha}$ to be the collection functions with domain $\alpha+1$ (ordered downwards) as follows. For each $n<\omega$ and $p_{n-1}<i \leq p_{n}$, let $\mathbb{S}_{\alpha}(i)$ consist of the empty set along with the collection of all functions $f$, satisfying

(1) $\operatorname{dom}(f)=[\beta, \alpha]$ for some $\beta \leq \alpha$;

(2) $f \uparrow\left[\beta, c_{\alpha}(n)\right] \in \mathbb{S}_{c_{\alpha}(n)}(m)$, where $m$ is such that $\mathbb{T}_{\alpha}(i)=\mathbb{T}_{c_{\alpha}(n)}(m)$; and

(3) $f \uparrow\left[c_{\alpha}(n)+1, \alpha\right]$ is the constant function with value $i$.

Then we set

$$
\mathbb{S}_{\alpha}=\bigcup_{i<\omega} \mathbb{S}_{\alpha}(i)
$$

There is a natural projection map $\psi_{\alpha}: \mathbb{S}_{\alpha} \rightarrow \mathbb{T}_{\alpha}$ such that for each $n<\omega$, $\psi_{\alpha}^{\prime \prime} \mathbb{S}_{\alpha}(n)=\mathbb{T}_{\alpha}(n)$. For $i<\omega, s \in \mathbb{S}_{\alpha}(i)$ and $n$ such that $p_{n-1}<i \leq p_{n}$, define $\psi_{\alpha}(s)=\psi_{c_{\alpha}(n)} \circ \sigma_{c_{\alpha}(n), \alpha}(s)$.

To build the reader's intuition, some structures are now displayed. The pattern of construction of $\mathbb{T}_{2}$ from $\mathbb{T}_{1}$ should help the reader construct a picture $\mathbb{T}_{3}$. Likewise, $\mathbb{S}_{1}$ and $\mathbb{S}_{2}$ provide enough for the reader to see the structure of $\mathbb{S}_{3}$. At limit stages, the tree $\mathbb{T}_{\omega}$ diagonalizes over the previous $\mathbb{T}_{n}, n<\omega$, whereas the structure $\mathbb{S}_{\omega}$ is a true extension of each $\mathbb{S}_{n}, n<\omega$. $\mathbb{T}_{\omega+1}$ and $\mathbb{S}_{\omega+1}$ are provided to help the reader see the continuation of the constructions after a limit ordinal.

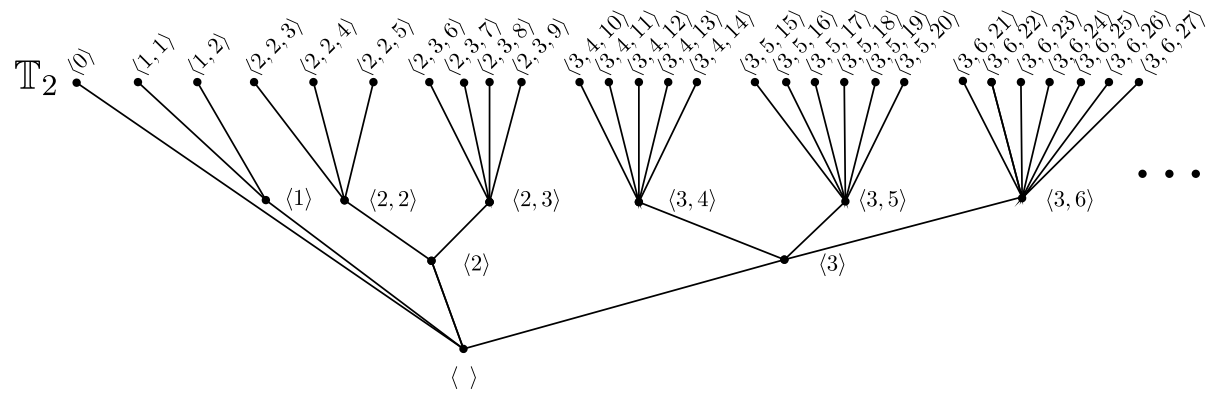

Figure 4 . The tree $\mathbb{T}_{2}$

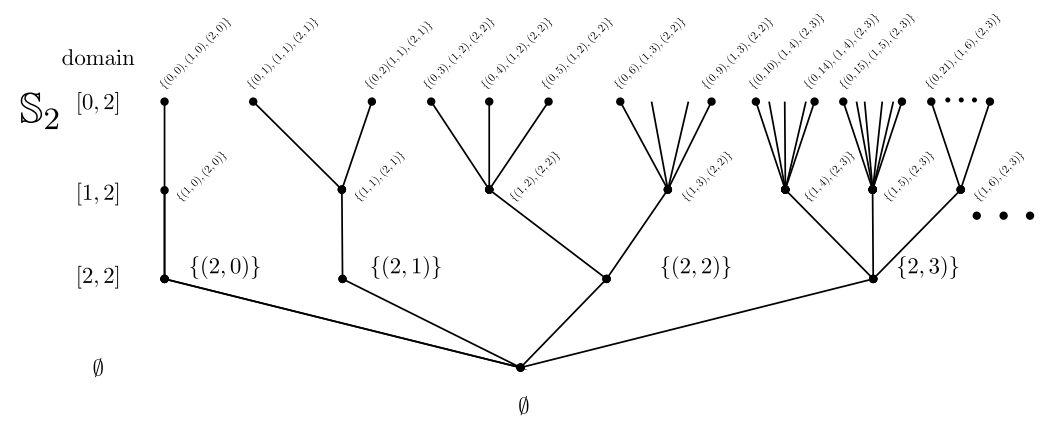

Figure 5. The tree $\mathbb{S}_{2}$ 


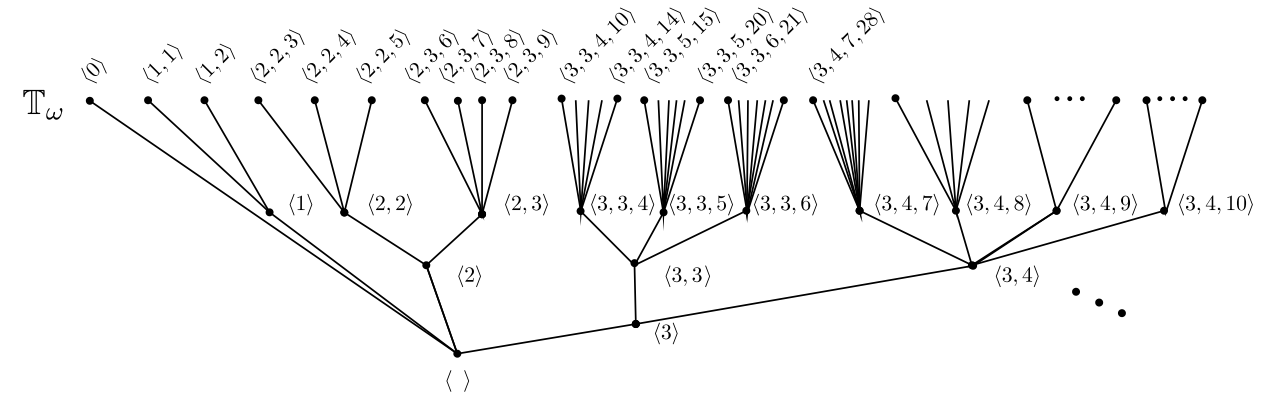

Figure 6 . The tree $\mathbb{T}_{\omega}$

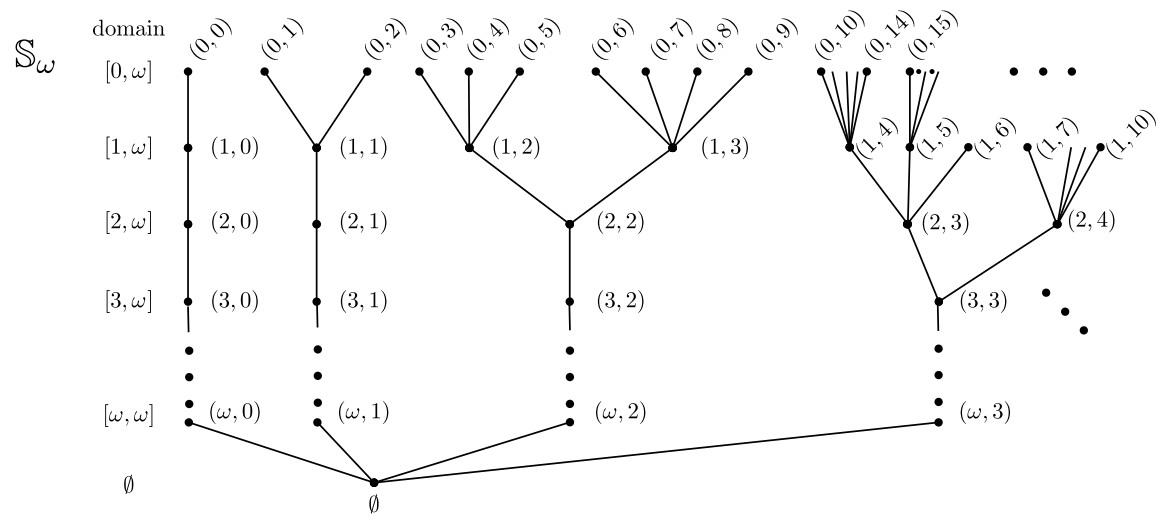

Figure 7 . The tree $\mathbb{S}_{\omega}$

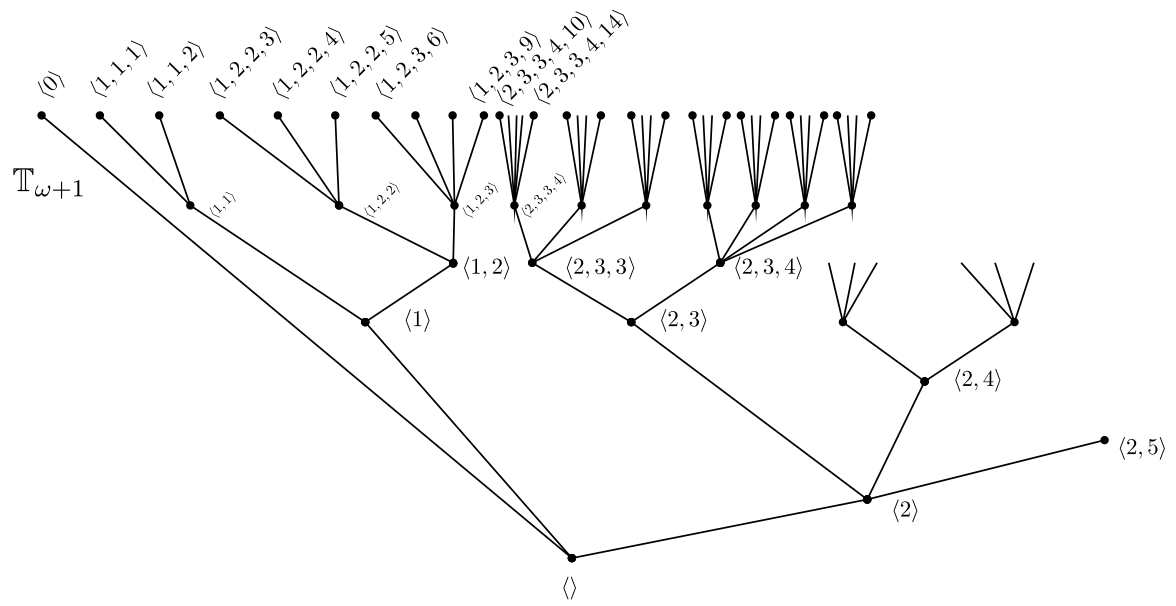

Figure 8 . The tree $\mathbb{T}_{\omega+1}$ 


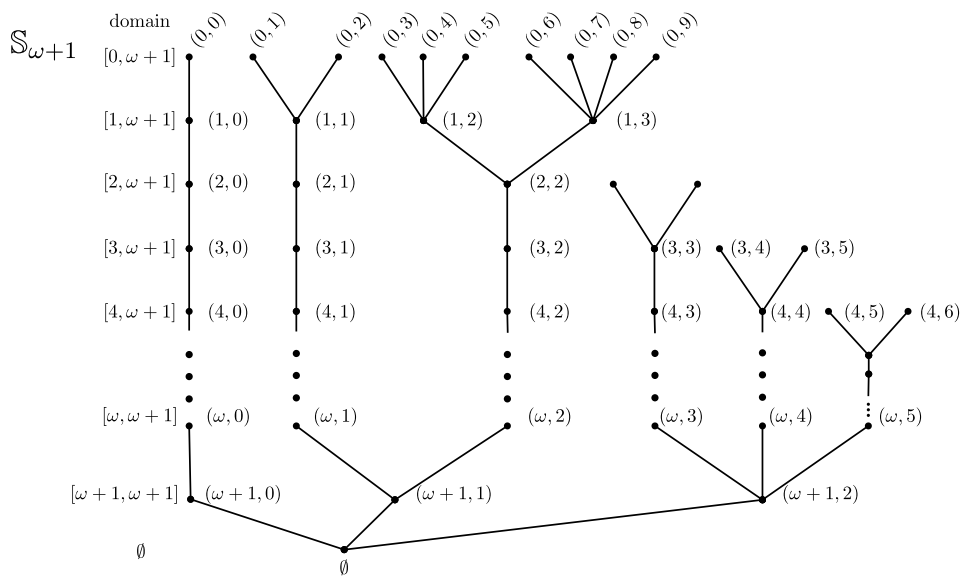

Figure 9. The tree $\mathbb{S}_{\omega+1}$

Recall that the nodes in the structures $\mathbb{S}_{\alpha}$ are functions with domain $[\beta, \alpha]$ for any $\beta \leq \alpha$, or else the empty function. The labels for the nodes in the above graphics for $\mathbb{S}_{\omega}$ and $\mathbb{S}_{\omega+1}$ had to be pared-down to fit on the page and are to be read as follows: The node in the figure represents the function consisting of that node and all nodes below it. For example, the node $(2,0)$ in Figure 7 represents the constant zero function with domain $[2, \omega]$, and the node $(0,4)$ in Figure 7 represents the function $\{(0,4),(1,2),(2,2),(3,2), \ldots,(\omega, 2)\}$. Likewise, in Figure 8, the node $(3,0)$ represents the constant zero function with domain $[3, \omega+1]$, and $(1,3)$ represents the function $\{(1,3),(2,2),(3,2),(4,2), \ldots,(\omega, 2),(\omega+1,1)\}$.

For each $\alpha<\omega_{1}$, if $s, s^{\prime} \in \mathbb{S}_{\alpha}, \operatorname{dom}(s)=[\beta, \alpha], \operatorname{dom}\left(s^{\prime}\right)=\left[\beta^{\prime}, \alpha\right]$, we say that $s^{\prime}$ is an immediate successor of $s$ iff $\beta=\beta^{\prime}+1$ and $s^{\prime} \supset s$; we also say that $s$ is the immediate predecessor of $s^{\prime}$. We shall say that $s$ is a splitting node iff $\beta$ is a successor ordinal, say $\beta=\gamma+1$, and there are $s_{0}, s_{1} \in \mathbb{S}_{\alpha}$ with $\operatorname{dom}\left(s_{0}\right)=\operatorname{dom}\left(s_{1}\right)=[\gamma, \alpha]$, $s_{0}\left\lceil[\beta, \alpha]=s_{1}\left\lceil[\beta, \alpha]=s\right.\right.$, and $s_{0} \neq s_{1}$ (that is, $s_{0}(\gamma) \neq s_{1}(\gamma)$ ).

Note that for each $t \in \mathbb{T}_{\alpha}, \psi_{\alpha}^{-1}(t)$ is a closed interval of $\mathbb{S}_{\alpha}(n)$ and the maximal node in $\psi_{\alpha}^{-1}(t)$ is either maximal in $\mathbb{S}_{\alpha}$ or else a splitting node in $\mathbb{S}_{\alpha}$. Whenever $s$ is a splitting node in $\mathbb{S}_{\alpha}, \min (\operatorname{dom}(s))$ must be a successor ordinal. This allows us to define the lexicographic ordering $<_{\operatorname{lex}}$ on $\mathbb{S}_{\alpha}$.

Definition 2.5. For $s, s^{\prime} \in \mathbb{S}_{\alpha}$, define $s<_{\operatorname{lex}} s^{\prime}$ iff either $s \subsetneq s^{\prime}$ (i.e. $s^{\prime}$ properly extends $s$, or else $s(\beta-1)<s^{\prime}(\beta-1)$, where $\beta \leq \alpha$ is the maximal ordinal such that $s \uparrow[\beta, \alpha]=s^{\prime} \uparrow[\beta, \alpha]$ and $s(\beta-1) \neq s^{\prime}(\beta-1)$. By an isomorphism between substructures of $\mathbb{S}_{\alpha}$, we mean a bijection which preserves the lexicographical order.

Remark 2.6. Each $\mathbb{S}_{\alpha}$ forms a tree-like structure. For $n<\omega, \mathbb{S}_{n}$ truly is a tree. For each $s \in \mathbb{S}_{\alpha},\left\{s^{\prime} \in \mathbb{S}_{\alpha}: s^{\prime} \subset s\right\}$ forms a linearly ordered set which is well-ordered by $\supset$. Moreover, for each $n<\omega$, there are only finitely many splitting nodes in $\mathbb{S}_{\alpha}(n)$. The $\mathbb{S}_{\alpha}$ may be viewed as the true structures, the trees $\mathbb{T}_{\alpha}$ being obtained by the simple projection mappings $\psi_{\alpha}: \mathbb{S}_{\alpha} \rightarrow \mathbb{T}_{\alpha}$. The map $\psi_{\alpha}$ essentially glues all nonsplitting nodes between two consecutive splitting nodes of $\mathbb{S}_{\alpha}$ to the upper splitting node. 
We are now equipped to define $\mathcal{R}_{\alpha}$.

Definition $2.7\left(\left(\mathcal{R}_{\alpha}, \leq_{\alpha}, r^{\alpha}\right), 1 \leq \alpha<\omega_{1}\right)$. A subset $X \subseteq \mathbb{T}_{\alpha}$ is a member of $\mathcal{R}_{\alpha}$ iff $\psi_{\alpha}^{-1}(X) \cong \mathbb{S}_{\alpha}$. Equivalently, $X \in \mathcal{R}_{\alpha}$ iff there is a strictly increasing sequence $\left(k_{n}\right)_{n<\omega}$ such that

(1) $X \cap \mathbb{T}_{\alpha}(m) \neq \emptyset$ iff $m=k_{n}$ for some $n<\omega ;$

(2) for each $n<\omega, \psi_{\alpha}^{-1}\left(X \cap \mathbb{T}_{\alpha}\left(k_{n}\right)\right) \cong \mathbb{S}_{\alpha}(n)$.

For the sequence $\left(k_{n}\right)_{n<\omega}$ above, we let $X(n)$ denote $X \cap \mathbb{T}_{\alpha}\left(k_{n}\right)$. We shall call $X(n)$ the $n$-th tree of $X$. For each $n<\omega$,

$$
\mathcal{R}_{\alpha}(n)=\left\{X(n): X \in \mathcal{R}_{\alpha}\right\} .
$$

For $n<\omega, r_{n}^{\alpha}(X)$ denotes $\bigcup_{i<n} X(i)$. The set of $n$-th approximations to members in $\mathcal{R}_{\alpha}$ is

$$
\mathcal{A R}_{n}^{\alpha}=\left\{r_{n}^{\alpha}(X): X \in \mathcal{R}_{\alpha}\right\},
$$

and the set of all finite approximations to members in $\mathcal{R}_{\alpha}$ is

$$
\mathcal{A R}^{\alpha}=\bigcup_{n<\omega} \mathcal{A R}_{n}^{\alpha}
$$

For $X, Y \in \mathcal{R}_{\alpha}$, define $Y \leq_{\alpha} X$ iff there is a strictly increasing sequence $\left(k_{n}\right)_{n<\omega}$ such that for each $n<\omega, Y(n) \subseteq X\left(k_{n}\right)$.

Let $a, b \in \mathcal{A R}^{\alpha}$ and $A, B \in \mathcal{R}_{\alpha}$. The quasi-ordering $\leq_{\text {fin }}^{\alpha}$ on $\mathcal{A R}^{\alpha}$ is defined as follows: $b \leq_{\text {fin }}^{\alpha} a$ if and only if there are $n \leq m$ such that $a \in \mathcal{A R}_{m}^{\alpha}, b \in \mathcal{A R}_{n}^{\alpha}$, and there is a strictly increasing sequence $\left(k_{i}\right)_{i<n}$ with $k_{n-1}<m$ such that for each $i<n, b(i)$ is a subtree of $a\left(k_{i}\right)$ (equivalently, $\left.b(i) \subseteq a\left(k_{i}\right)\right)$. In fact, $\leq_{\text {fin }}^{\alpha}$ is a partial ordering. We write $a \leq_{\text {fin }}^{\alpha} B$ if and only if there is an $A \in \mathcal{R}_{\alpha}$ such that $a \sqsubset A$ and $A \leq_{\alpha} B . B / a$ is defined to be $\bigcup\left\{B(n): n \geq \operatorname{depth}_{B}(a)\right\}$. The basic open sets are given by

$$
[a, B]=\left\{X \in \mathcal{R}_{\alpha}: a \sqsubseteq X \text { and } X \leq_{\alpha} B\right\} .
$$

Remark 2.8. Since the quasi-ordering $\leq_{\text {fin }}^{\alpha}$ is actually a partial ordering, it follows from Corollary 5.19 in [15] that for any front $\mathcal{F}$ on $[0, X], X \in \mathcal{R}_{\alpha}$, there is a $Y \leq_{\alpha} X$ for which $\mathcal{F} \mid Y$ is a barrier.

We point out the following trivial but useful facts.

Fact 2.9. (1) For $u \subseteq \mathbb{T}_{\alpha}, u \in \mathcal{R}_{\alpha}(n)$ iff $\psi_{\alpha}^{-1}(u) \subseteq \mathbb{S}_{\alpha}(m)$ for some $m \geq n$ and $\psi_{\alpha}^{-1}(u) \cong \mathbb{S}_{\alpha}(n)$.

(2) $u \in \mathcal{R}_{\alpha}(n)$ iff the structure obtained by identifying each node $t$ in $u$ which is both not a leaf and not a splitting node in $u$ with the minimal splitting node in $u$ above $t$, is isomorphic to $\mathbb{T}_{\alpha}(n)$.

(3) Because of the structure inherent in being a member of $\mathcal{R}_{\alpha}$, the following are equivalent for all $X, Y \in \mathcal{R}_{\alpha}$ :

(a) $Y \leq_{\alpha} X$.

(b) There is a strictly increasing sequence $\left(k_{n}\right)_{n<\omega}$ such that for each $n<$ $\omega, Y(n)$ is a subtree of $X\left(k_{n}\right), \psi_{\alpha}^{-1}(Y(n))$ is isomorphic to $\mathbb{S}_{\alpha}(n)$, and $\psi_{\alpha}^{-1}(Y(n))$ is a substructure of $\psi_{\alpha}^{-1}\left(X\left(k_{n}\right)\right)$.

(c) $Y \subseteq X$. 
Throughout this paper, we use the following fact without further mention.

Fact 2.10. Suppose $1 \leq \alpha<\omega_{1}, n<\omega, a \in \mathcal{A R}_{n}^{\alpha}, B \in \mathcal{R}_{\alpha}$, and there are $k<k^{\prime}$ such that $B(n) \subseteq \mathbb{T}_{\alpha}\left(k^{\prime}\right)$ and $a(n-1) \subseteq \mathbb{T}_{\alpha}(k)$. Then $a \cup\left(B / r_{n}^{\alpha}(B)\right)$ is a member of $\mathcal{R}_{\alpha}$.

\section{3. $\mathcal{R}_{\alpha}$ IS A TOPOLOGICAL RAMSEY SPACE, FOR EACH $\alpha<\omega_{1}$}

In this section, we prove by induction that each $\mathcal{R}_{\alpha}, 2 \leq \alpha<\omega_{1}$, is a topological Ramsey space. In the process, we define the canonical equivalence relations on $\mathcal{R}_{\alpha}(n)$ and on $\mathcal{A R}_{n}^{\alpha}$. Recall that $\mathcal{R}_{0}$ denotes the Ellentuck space, which is the fundamental example of a topological Ramsey space. In Theorem 3.9 of $\left[\underline{5}, \mathcal{R}_{1}\right.$ was shown to be a topological Ramsey space. This forms the basis of the induction scheme which cycles through this and the next section. We begin this section by setting the stage for the introduction of the canonical equivalence relations.

A subset $S \subseteq \mathbb{S}_{\alpha}$ is called downward closed iff $\emptyset \in S$ and, for all $s \in S$, if $\operatorname{dom}(s)=[\beta, \alpha]$, then also $s \uparrow[\gamma, \alpha] \in S$ for all $\gamma \in[\beta, \alpha]$. Two downward closed sets $S, S^{\prime} \subseteq \mathbb{S}_{\alpha}$ are isomorphic iff there is a bijection between $S$ and $S^{\prime}$ which preserves the lexicographic ordering.

Definition 3.1. For each $n<\omega$, define $\mathfrak{S}_{\alpha}(n)$ to be the collection of all nonempty downward closed subsets of $\mathbb{S}_{\alpha}(n)$. For each $n \leq m, \mathcal{R}_{\alpha}(n) \mid \mathbb{T}_{\alpha}(m)$ denotes the collection of all $u \in \mathcal{R}_{\alpha}(n)$ such that $u \subseteq \mathbb{T}_{\alpha}(m)$. Define $S \in \mathfrak{S}_{\alpha}(n, m)$ iff $S \in \mathfrak{S}_{\alpha}(n)$ and there is a $u \in \mathcal{R}_{\alpha}(n) \mid \mathbb{T}_{\alpha}(m)$ and a nonempty subtree $v \subseteq u$ such that $S \cong \psi_{\alpha}^{-1}(v)$.

We point out the following. The set $\{\emptyset\}$ is the $\subseteq$-minimal member of each $\mathfrak{S}_{\alpha}(n)$; $\{\langle\rangle\}$ is the smallest nonempty subtree of any member in $\mathcal{R}_{\alpha}(n) \cdot \psi_{\alpha}^{-1}(v)=\{\emptyset\}$ iff $v=\{\langle\rangle\}$. Note that $\mathfrak{S}_{\alpha}(n, m)$ is finite, for all $n \leq m$. However, if $\alpha$ is infinite, then $\mathfrak{S}_{\alpha}(n)$ is countably infinite.

Given $\beta \leq \alpha$, we shall let $S_{\beta}^{\alpha}$, or just $S_{\beta}$, denote the member of $\mathfrak{S}_{\alpha}(0)$ which is a downward closed chain of order type $[\beta, \alpha+1]$. Thus, $S_{\beta}=\mathbb{S}_{\alpha}(0) \uparrow[\beta, \alpha+1]$, which is the collection of all constantly zero functions on domains $[\gamma, \alpha]$, for $\beta \leq \gamma \leq \alpha$, along with the empty function.

Examples 3.2. $\mathfrak{S}_{2}(1,1)$ consists of all subtrees of $\mathbb{S}_{2}(1)$ except for $\{\{(2,1), \emptyset\}\}$; that is, the members of $\mathfrak{S}_{2}(1,1)$ are $\mathbb{S}_{2}(1) ;\{\{(0,1),(1,1),(2,1)\},\{(1,1),(2,1)\}$, $\{(2,1)\}, \emptyset\} ; \quad\{\{(0,2),(1,1),(2,1)\},\{(1,1),(2,1)\}, \quad\{(2,1)\}, \emptyset\} ; \quad\{\{(1,1),(2,1)\}$, $\{(2,1)\}, \emptyset\} ;\{\{(2,1)\}, \emptyset\} ;$ and $\{\emptyset\} . \mathfrak{S}_{2}(1,2)=\mathfrak{S}_{2}(1,1) \cup\{\{(2,1), \emptyset\}\}$. Note that $\mathfrak{S}_{2}(1)=\mathfrak{S}_{2}(1,2)$.

$\mathfrak{S}_{\omega}(0,0)=\left\{S_{0}^{\omega},\{\emptyset\}\right\}, \mathfrak{S}_{\omega}(0,1)=\left\{S_{0}^{\omega}, S_{1}^{\omega},\{\emptyset\}\right\}, \ldots$, and in general, $\mathfrak{S}_{\omega}(0, m)=$ $\left\{S_{0}^{\omega}, \ldots, S_{m}^{\omega}\right\} \cup\{\{\emptyset\}\} . \mathfrak{S}_{\omega}(0)=\bigcup_{m<\omega} \mathfrak{S}_{\omega}(0, m) \cup\{\{(\omega, 0), \emptyset\}\}=\left\{S_{k}^{\omega}: k \leq \omega\right\}$.

The next fact follows immediately from Definition 3.1

Fact 3.3. Let $n \leq m<m^{\prime}$. Then:

(1) $\mathfrak{S}_{\alpha}(n, m) \subseteq \mathfrak{S}_{\alpha}\left(n, m^{\prime}\right) \subseteq \mathfrak{S}_{\alpha}(n)$.

(2) $\mathfrak{S}_{\alpha}(n)=\bigcup\left\{\mathfrak{S}_{\alpha}(n, m): m \geq n\right\} \cup\{\{(\alpha, n), \emptyset\}\}$.

Next we define projection maps $\pi_{S}$. The map $\pi_{S}$ takes a structure $u$ in its domain and projects it to the substructure of $u$ whose $\psi_{\alpha}$-preimage is isomorphic to $S$. 
Definition 3.4. Let $1 \leq \alpha<\omega_{1}$ and $m<\omega$ be given. Let $S \in \mathfrak{S}_{\alpha}(m)$. Define $\pi_{S}$ on $\mathcal{R}_{\alpha}(m)$ as follows: Given $u \in \mathcal{R}_{\alpha}(m)$, let $\iota_{u}: \mathbb{S}_{\alpha}(m) \rightarrow \psi_{\alpha}^{-1}(u)$ be the isomorphism from $\mathbb{S}_{\alpha}(m)$ to $\psi_{\alpha}^{-1}(u)$. Define

$$
\pi_{S}(u)=\psi_{\alpha} \circ \iota_{u}(S) \text {. }
$$

Given $n<m$, letting $S$ be the subset of $\mathbb{S}_{\beta}(m)$ which consists of the lexicographically least (i.e. leftmost) members of $\mathbb{S}_{\alpha}(m)$ which together comprise a set isomorphic to $\mathbb{S}_{\alpha}(n)$, let $\pi_{m, n}^{\alpha}$ denote $\pi_{S}$ for this particular $S$.

Note that if $n<m$ and $S$ is any downward closed subset of $\mathbb{S}_{\alpha}(m)$ such that $S$ is isomorphic to $\mathbb{S}_{\alpha}(n)$, then $\pi_{S}$ is in fact a map from $\mathcal{R}_{\alpha}(m)$ to $\mathcal{R}_{\alpha}(n)$.

We now introduce the various canonical equivalence relations.

Definition 3.5 (Canonical equivalence relations on $\mathcal{R}_{\alpha}(n)$, for $\alpha<\omega_{1}$ ). For each $n<\omega$, each $S \in \mathfrak{S}_{\alpha}(n)$ induces the equivalence relation $\mathrm{E}_{S}$ on $\mathcal{R}_{\alpha}(n)$ defined as follows: For $u, v \in \mathcal{R}_{\alpha}(n)$,

$$
u \mathrm{E}_{S} v \Leftrightarrow \pi_{S}(u)=\pi_{S}(v) .
$$

Let $\mathcal{E}_{\alpha}(n)$ denote the collection of all equivalence relations of the form $\mathrm{E}_{S}$, where $S \in \mathfrak{S}_{\alpha}(n) . \mathcal{E}_{\alpha}(n)$ is the set of canonical equivalence relations on $\mathcal{R}_{\alpha}(n)$.

Definition 3.6 (Canonical equivalence relations on $\mathcal{R}_{\alpha}(n) \mid X(m)$, for $\alpha<\omega_{1}$, $X \in \mathcal{R}_{\alpha}$, and $\left.n \leq m<\omega\right)$. Given any $\alpha<\omega_{1}, X \in \mathcal{R}_{\alpha}$, and $n \leq m$, the canonical equivalence relations on $\mathcal{R}_{\alpha}(n) \mid X(m)$ are given by $\mathrm{E}_{S}$, where $S \in \mathfrak{S}_{\alpha}(n, m)$.

Remark 3.7. For any $n \leq m$ and any $S \in \mathfrak{S}_{\alpha}(n)$, there is an $S^{\prime} \in \mathfrak{S}_{\alpha}(n, m)$ such that $\mathrm{E}_{S}$ is the same as $\mathrm{E}_{S^{\prime}}$ when restricted to $\mathcal{R}_{\alpha}(n) \mid X(m)$. Moreover, this $S^{\prime}$ is unique, and it must be the case that $S \subseteq S^{\prime}$.

Definition 3.8 (Canonical equivalence relations on $\mathcal{A R}_{n}^{\alpha}$ ). For any given $n_{0}<$ $n_{1}<\omega$ and $X \in \mathcal{R}_{\alpha}$, let $X\left[n_{0}, n_{1}\right)=\bigcup\left\{X(n): n_{0} \leq n<n_{1}\right\}$. Let

$$
\mathcal{R}_{\alpha}\left[n_{0}, n_{1}\right)=\left\{X\left[n_{0}, n_{1}\right): X \in \mathcal{R}_{\alpha}\right\} .
$$

We shall say that an equivalence relation $\mathrm{E}$ on $\mathcal{R}_{\alpha}\left(\left[n_{0}, n_{1}\right)\right)$ is canonical iff there are $S(i) \in \mathfrak{S}_{\alpha}(i), n_{0} \leq i<n_{1}$, such that for all $x, y \in \mathcal{R}_{\alpha}\left(\left[n_{0}, n_{1}\right)\right)$,

$$
x \mathrm{E} y \Leftrightarrow \forall n_{0} \leq i<n_{1}, x(i) \mathrm{E}_{S(i)} y(i) .
$$

Taking $n_{0}=0$ and $n_{1}=n$, this defines the canonical equivalence relations on $\mathcal{A R}_{n}^{\alpha}$, for all $\alpha<\omega_{1}$.

Numbers of canonical equivalence relations. For each $k, n<\omega$, the number of canonical equivalence relations on $\mathcal{R}_{k}(n)$ and $\mathcal{A R}_{n}^{k}$ are given by a recursive formula. Let $N_{k}(n)$ denote the number of canonical equivalence relations on $\mathcal{R}_{k}(n)$. Recall from [5] that for each $n, N_{1}(n)=2^{n+1}+1$, and for $n \geq 1$, there are $\Pi_{i<n}\left(2^{i+1}+1\right)$ canonical equivalence relations on $\mathcal{A R}_{n}^{1}$. It will be proved in Section 4 that the canonical equivalence relations on $\mathcal{R}_{\alpha}(n)$ and $\mathcal{A R}_{n}^{\alpha}$ are precisely the ones defined above. Hence, for $k \geq 1$,

$$
N_{k+1}(n)= \begin{cases}N_{k}(n)+1 & \text { if } n \leq k \\ \left(\prod_{l_{n}^{k} \leq j<l_{n+1}^{k}}^{k} N_{k}(j)\right)+1 & \text { if } n>k .\end{cases}
$$

For $n \geq 1$, there are $\Pi_{i<n} N_{k}(i)$ many canonical equivalence relations on $\mathcal{A R}_{k}^{n}$.

Thus, for $k=2$, there are 4 canonical equivalence relations on $\mathcal{R}_{2}(0), 6$ canonical equivalence relations on $\mathcal{R}_{2}(1), 154$ canonical equivalence relations on $\mathcal{R}_{2}(2)$, 
etc. There are 4 canonical equivalence relations on $\mathcal{A R}_{1}^{\alpha}, 24$ canonical equivalence relations on $\mathcal{A R}_{2}^{\alpha}, 3696$ canonical equivalence relations on $\mathcal{A R}_{3}^{2}$, etc.

For $\omega \leq \alpha<\omega_{1}$ and $n \leq m, \mathfrak{S}_{\alpha}(n, m)$ is finite; however, $\mathfrak{S}_{\alpha}(n)$ is countably infinite.

The following theorem for $\mathcal{R}_{1}$ was proved in [5]. Recall that $\mathcal{A R}_{n}^{1} \mid D$ denotes the collection of all $a \in \mathcal{A R}_{n}^{1}$ such that $a \leq_{\text {fin }}^{1} D$.

Theorem 3.9 (Canonization Theorem for $\mathcal{A R}_{n}^{1}$ [5]). Let $1 \leq n<\omega$. Given any $A \in \mathcal{R}_{1}$ and any equivalence relation $\mathrm{R}$ on $\mathcal{A R}_{n}^{1} \mid A$, there is a $D \leq_{1} A$ such that $\mathrm{R}$ is canonical on $\mathcal{A R}_{n}^{1} \mid D$.

Theorem 3.9 serves as the basis for the following Inductive Scheme: Given Theorem 3.9 we prove Theorem 3.10 and Lemma 3.11 for $\beta=1$. These are then used to prove Theorems 3.12, 3.13 and 3.14 for for $\alpha=2$. Given these theorems, we then prove Theorems 4.12 and 4.22 in Section 4 for $\alpha=2$. The induction scheme continues for $3 \leq \alpha<\omega_{1}$ as follows. Assume Theorems 4.22 and 3.12 hold for all $1 \leq \beta<\alpha$. If $\alpha$ is a successor ordinal, say $\alpha=\beta+1$, then we also assume Theorem 3.10 and Lemma 3.11 hold for all $1 \leq \gamma<\beta$, and we prove Theorem 3.10 and Lemma 3.11 hold for $\beta$. If $\alpha$ is a limit ordinal, then by the time we have proved Theorems 4.22 and 3.12 for all $1 \leq \beta<\alpha$, we will also have proved Theorem 3.10 and Lemma 3.11 for all $1 \leq \beta<\alpha$. These are then used to prove Theorems 3.12 , 3.13. and 3.14 for $\alpha$, so that in particular, $\mathcal{R}_{\alpha}$ is a topological Ramsey space. Then we prove Theorems 4.12 and 4.22 for $\alpha$ in Section 4 .

Thus, let $1<\alpha<\omega_{1}$. In order to prove that $\mathcal{R}_{\alpha}$ is a topological Ramsey space, we will need to show that the pigeonhole principal A.4 holds for $\mathcal{R}_{\alpha}(n)$, for each $n<\omega$. Toward this end, we first prove some finite canonization theorems. The next theorem follows from Theorem 3.9 for $\beta=1$; for $\beta \geq 2$, it follows from Theorem 4.22 for $\beta$. We omit the proof, as it is completely analogous to the standard proof of the Finite Ramsey Theorem from the Infinite Ramsey Theorem.

Theorem 3.10 (Finite Canonization Theorem for $\mathcal{A R}_{n}^{\beta}$ ). For each $n \leq k<\omega$ and each $X \in \mathcal{R}_{\beta}$, there is an $m<\omega$ such that for each equivalence relation $\mathrm{E}$ on $\mathcal{A R}_{n}^{\beta} \mid r_{m}^{\beta}(X)$, there is an $a \in \mathcal{A R}_{k}^{\beta} \mid r_{m}^{\beta}(X)$ such that $\mathrm{E}$ is canonical on $\mathcal{A R}_{n}^{\beta} \mid a$.

Lemma 3.11. Let $n_{0}<n_{1}$ and $k_{0}<k_{1}$ be such that $k_{0} \geq n_{0}$ and $k_{1}-k_{0} \geq n_{1}-n_{0}$, and let $X \in \mathcal{R}_{\beta}$. There is an $m$ such that for each equivalence relation $\mathrm{E}$ on $\mathcal{R}_{\beta}\left[n_{0}, n_{1}\right) \mid r_{m}^{\beta}(X)$, there is a $y \in \mathcal{R}^{\beta}\left[k_{0}, k_{1}\right) \mid r_{m}^{\beta}(X)$ such that $\mathrm{E}$ is canonical on $\mathcal{R}_{\beta}\left[n_{0}, n_{1}\right) \mid y$.

Proof. Let $n_{0}, n_{1}, k_{0}, k_{1}$ be as in the hypotheses. Take $m$ from Theorem 3.10 for $n_{1}$ and $k_{1}$. Let $\mathrm{E}$ be an equivalence relation on $\mathcal{R}^{\beta}\left[n_{0}, n_{1}\right) \mid r_{m}^{\beta}(X)$. Define an equivalence relation $\mathrm{E}^{\prime}$ on $\mathcal{A} \mathcal{R}_{n_{1}}^{\beta} \mid r_{m}^{\beta}(X)$ by defining $a \mathrm{E}^{\prime} b$ if and only if $a\left[n_{0}, n_{1}\right) \mathrm{E} b\left[n_{0}, n_{1}\right)$, for $a, b \in \mathcal{A R}_{n_{1}}^{\beta} \mid r_{m}^{\beta}(X)$. Then there is a $c \in \mathcal{A R}_{k_{1}}^{\beta} \mid r_{m}^{\beta}(X)$ such that $\mathrm{E}^{\prime}$ is canonical on $\mathcal{A R}_{n_{1}}^{\beta} \mid c$. Hence, E is canonical on $\mathcal{R}^{\beta}\left[n_{0}, n_{1}\right) \mid c\left[k_{0}, k_{1}\right)$.

Theorem 3.12 (Finite Canonization Theorem for $\mathcal{R}_{\alpha}(n)$ ). Let $n \leq k<\omega$ and $X \in \mathcal{R}_{\alpha}$ be given. Then there is an $m$ such that for each equivalence relation $\mathrm{E}$ on $\mathcal{R}_{\alpha}(n) \mid X(m)$, there is a $y \in \mathcal{R}_{\alpha}(k) \mid X(m)$ such that $\mathrm{E}$ is canonical on $\mathcal{R}_{\alpha}(n) \mid y$.

Proof. Let $n, k, X$ be as in the hypotheses. There are two cases. 
Case 1. $\alpha$ is a successor ordinal.

Let $\beta$ be such that $\alpha=\beta+1$. Let $n_{0}=l_{n}^{\beta}, n_{1}=l_{n+1}^{\beta}, k_{0}=l_{k}^{\beta}$, and $k_{1}=l_{k+1}^{\beta}$. Take $m_{0}$ from Lemma 3.11. Let $m$ be large enough that $l_{m+1}^{\beta}-l_{m}^{\beta}>m_{0}$. Let E be an equivalence relation on $\mathcal{R}_{\alpha}(n) \mid X(m)$. Take $a \in \mathcal{A R}_{m_{0}}^{\beta}$ such that $a \subseteq \tau_{\beta, \alpha}^{\prime \prime} X(m)$. Let $\mathrm{E}^{\prime}$ be the equivalence relation on $\mathcal{R}_{\beta}\left[n_{0}, n_{1}\right) \mid a$ induced by $\mathrm{E}$ in the following manner: For all $u^{\prime}, v^{\prime} \in \mathcal{R}_{\beta}\left[n_{0}, n_{1}\right) \mid a, u^{\prime} \mathrm{E}^{\prime} v^{\prime}$ iff $u \mathrm{E} v$, where $u=\{\langle\rangle\} \cup\left\{\langle m\rangle \frown t: t \in u^{\prime}\right\}$ and $v=\{\langle\rangle\} \cup\left\{\langle m\rangle \frown t: t \in v^{\prime}\right\}$. By Lemma 3.11, there is a $y^{\prime} \in \mathcal{A R}_{k_{1}}^{\beta} \mid a$ such that $\mathrm{E}^{\prime}$ is canonical on $\mathcal{R}_{\beta}\left[n_{0}, n_{1}\right) \mid y^{\prime}\left[k_{0}, k_{1}\right)$, given by some $S(i) \in \mathfrak{S}_{\beta}(i), n_{0} \leq i<n_{1}$. Letting $y=\{\langle\rangle\} \cup\left\{\langle m\rangle \frown t: t \in y^{\prime}\left[k_{0}, k_{1}\right)\right\}$, we have that $y \in \mathcal{R}_{\alpha}(k)$. Moreover, $\mathrm{E}$ is canonical on $\mathcal{R}_{\alpha}(n) \mid y$, given by $\mathrm{E}_{S}$, where if at least one $S(i) \neq\{\emptyset\}$, then we let $S=\{\emptyset\} \bigcup\left\{s \cup\{(\alpha, n)\}: n_{0} \leq i<n_{1}, s \in S(i)\right\}$, and if all $S(i)=\{\emptyset\}$, then $S=\{\emptyset\}$.

Case 2. $\alpha$ is a limit ordinal.

Let $\gamma \leq \delta$ and $n_{\gamma}, k_{\delta}$ be the ordinals such that $\mathbb{T}_{\alpha}(n)=\mathbb{T}_{\gamma}\left(n_{\gamma}\right)$ and $\mathbb{T}_{\alpha}(k)=$ $\mathbb{T}_{\delta}\left(k_{\delta}\right)$, by construction of $\mathbb{T}_{\alpha} \cdot \mathbb{T}_{\gamma}\left(n_{\gamma}\right)$ is contained in $\tau_{\gamma, \delta}^{\prime \prime} \mathbb{T}_{\delta}\left(n_{\delta}\right)$, for some $n_{\delta} \leq k_{\delta}$. Note that necessarily $n_{\delta} \leq k_{\delta}$. Take $S \in \mathfrak{S}_{\delta}\left(n_{\delta}\right)$ which satisfies $\tau_{\gamma, \delta} \circ \pi_{S}\left(\mathbb{T}_{\delta}\left(n_{\delta}\right)\right)=$ $\mathbb{T}_{\gamma}\left(n_{\gamma}\right)$. Take $k^{\prime}$ large enough that for any $w \in \mathcal{R}_{\delta}\left(k^{\prime}\right)$, there is some member $v \in \mathcal{R}_{\delta}\left(k_{\delta}\right) \mid w$ such that as $u$ ranges over $\mathcal{R}_{\delta}\left(n_{\delta}\right) \mid w$, their projections $\tau_{\gamma, \delta} \circ \pi_{S}(u)$ range over (and possibly beyond) $\mathcal{R}_{\gamma}\left(n_{\gamma}\right) \mid v$. By Theorem 3.12 for $\mathcal{R}_{\delta}$, there is an $m$ such that for each $x \in \mathcal{R}_{\delta}(m)$ and equivalence relation $\mathrm{E}^{\prime}$ on $\mathcal{R}_{\delta}\left(n_{\delta}\right) \mid x$, there is a $w \in \mathcal{R}_{\delta}\left(k^{\prime}\right) \mid x$ such that $\mathrm{E}^{\prime}$ is canonical on $\mathcal{R}_{\delta}\left(n_{\delta}\right) \mid w$.

This $m$ works for $\mathcal{R}_{\alpha}$ : Let $X \in \mathcal{R}_{\alpha}$ and take any equivalence relation $\mathrm{E}$ on $\mathcal{R}_{\alpha}(n) \mid X(m)$. Take $x \subseteq \tau_{\delta, \alpha}(X(m))$ such that $x \in \mathcal{R}_{\delta}(m)$. Let stem $(x)$ denote the collection of all $t \in X(m)$ which are strictly below all nodes in $x$. Note that $\operatorname{stem}(x)$ is a downward closed interval in $X(m)$. Define $\mathrm{E}^{\prime}$ to be the equivalence relation on $\mathcal{R}_{\delta}\left(n_{\delta}\right) \mid x$ as follows. For $y \in \mathcal{R}_{\delta}\left(n_{\delta}\right)$, let $\bar{y}$ denote the member of $\mathcal{R}_{\alpha}(n)$ for which $\tau_{\delta, \alpha}(\bar{y})=y$. For $y, z \in \mathcal{R}_{\delta}\left(n_{\delta}\right) \mid x$, define $y \mathrm{E}^{\prime} z$ iff $\bar{y} \mathrm{E} \bar{z}$. By Theorem 3.12, there is a $w \in \mathcal{R}_{\delta}\left(k^{\prime}\right) \mid x$ such that $\mathrm{E}^{\prime}$ is canonical on $\mathcal{R}_{\delta}\left(n_{\delta}\right) \mid w$. By our choice of $k^{\prime}$, there is some member $v \in \mathcal{R}_{\delta}\left(k_{\delta}\right) \mid w$ such that as $u$ ranges over $\mathcal{R}_{\delta}\left(n_{\delta}\right) \mid w$, their projections $\tau_{\gamma, \delta} \circ \pi_{S}(u)$ range over (and possibly beyond) $\mathcal{R}_{\gamma}\left(n_{\gamma}\right) \mid v$. Let $\bar{v}=v \cup \operatorname{stem}(x)$. Then $\bar{v} \in \mathcal{R}_{\alpha}(k)$, and $\mathrm{E}$ is canonical on $\mathcal{R}_{\alpha}(n) \mid \bar{v}$.

Theorem 3.13 (Finite version of the pigeonhole principle for $\mathcal{R}_{\alpha}(n)$ ). Let $n \leq$ $k<\omega$ and $X \in \mathcal{R}_{\alpha}$ be given. Then there is an $m$ such that for each 2-coloring $f: \mathcal{R}_{\alpha}(n) \mid X(m) \rightarrow 2$, there is a $y \in \mathcal{R}_{\alpha}(k) \mid X(m)$ such that $f$ is monochromatic on $\mathcal{R}_{\alpha}(n) \mid y$.

Proof. Let $n, k, X$ be as in the hypotheses. Take $m$ from Theorem 3.12. Then there is a $y \in \mathcal{R}_{\alpha}(k) \mid X(m)$ such that the equivalence relation induced by $f$ is canonical on $\mathcal{R}_{\alpha}(n) \mid y$. But the only canonical equivalence relation induced by a 2 -coloring is the trivial one. Thus, $f$ is monochromatic on $\mathcal{R}_{\alpha}(n) \mid y$.

Theorem 3.14. $\left(\mathcal{R}_{\alpha}, \leq_{\alpha}, r^{\alpha}\right)$ is a topological Ramsey space.

Proof. By the Abstract Ellentuck Theorem, it suffices to show that $\left(\mathcal{R}_{\alpha}, \leq_{\alpha}, r^{\alpha}\right)$ is a closed subspace of the Tychonoff power $\left(\mathcal{A} \mathcal{R}^{\alpha}\right)^{\mathbb{N}}$ of $\mathcal{A} \mathcal{R}^{\alpha}$ with its discrete topology, and that $\left(\mathcal{R}_{\alpha}, \leq_{\alpha}, r^{\alpha}\right)$ satisfies axioms A.1 - A.4 (see [5]).

$\mathcal{R}_{\alpha}$ is identified with the subspace of $\left(\mathcal{A} \mathcal{R}^{\alpha}\right)^{\mathbb{N}}$ consisting of all sequences $\left\langle a_{n}\right.$ : $n<\omega\rangle$ such that there is an $A \in \mathcal{R}_{\alpha}$ such that for each $n<\omega, a_{n}=r_{n}^{\alpha}(A)$. That 
$\mathcal{R}_{\alpha}$ is a closed subspace of $\left(\mathcal{A} \mathcal{R}^{\alpha}\right)^{\mathbb{N}}$ follows from the fact that given any sequence $\left\langle a_{n}: n<\omega\right\rangle$ such that each $a_{n} \in \mathcal{A R}_{n}^{\alpha}$ and $r_{n}^{\alpha}\left(a_{k}\right)=a_{n}$ for each $k \geq n$, the union $A=\bigcup_{n<\omega} a_{n}$ is a member of $\mathcal{R}_{\alpha}$. A.1 and A.2 are trivially satisfied, by the definition of $\mathcal{R}_{\alpha}$.

A.3. (1) If $\operatorname{depth}_{B}(a)=n<\infty$, then $a \leq_{\text {fin }}^{\alpha} r_{n}^{\alpha}(B)$. If $A \in\left[\operatorname{depth}_{B}(a), B\right]$, then $r_{n}^{\alpha}(A)=r_{n}^{\alpha}(B)$, and for each $k \geq n$, there is an $m_{k}$ such that $A(k) \subseteq B\left(m_{k}\right)$. Letting $l$ be such that $a \in \mathcal{A R}_{l}^{\alpha}$, for each $i \geq 1$, let $w(l+i)$ be any subtree of $A(n+i)$ such that $\psi_{\alpha}^{-1}(w(l+i)) \cong \mathbb{S}_{\alpha}(l+i)$. Let $A^{\prime}=a \cup \bigcup\{w(l+i): i \geq 1\}$. Then $A^{\prime} \in[a, A]$, so $[a, A] \neq \emptyset$.

(2) Suppose $A \leq_{\alpha} B$ and $[a, A] \neq \emptyset$. Then $\operatorname{depth}_{B}(a)<\infty$ since $A \leq_{\alpha} B$. Let $n=\operatorname{depth}_{B}(a)$ and $k=\operatorname{depth}_{A}(a)$. Note that $k \leq n$, and for each $j \geq k$, $A(j) \subseteq B(l)$ for some $l \geq n$. Let $A^{\prime}=r_{n}^{\alpha}(B) \cup \bigcup\{\bar{A}(n+i): i<\omega\}$. Then $A^{\prime} \in\left[\operatorname{depth}_{B}(a), B\right]$ and $\emptyset \neq\left[a, A^{\prime}\right] \subseteq[a, A]$.

A.4. Suppose that $\operatorname{depth}_{B}(a)=n<\infty$ and $\mathcal{O} \subseteq \mathcal{A R}_{|a|+1}^{\alpha}$. Let $k=|a|$. (Recall that $r_{k+1}^{\alpha}[a, B]$ is defined to be the collection of $c \in \mathcal{A R}_{k+1}^{\alpha}$ such that $r_{k}^{\alpha}(c)=r_{k}^{\alpha}(a)$ and $c(k)$ is a subtree of $B(m)$ for some $m \geq n$.) So we may think of $\mathcal{O}$ as a 2-coloring on the collection of subtrees $u \subseteq B(m), m \geq n$, such that $\psi_{\alpha}^{-1}(u) \cong \mathbb{S}_{\alpha}(k)$. Say a set $u \in \mathcal{R}_{\alpha}(k) \mid B / r_{n}^{\alpha}(B)$ has color 0 if $a \cup u$ is in $\mathcal{O}$ and has color 1 if $a \cup u$ is in $\mathcal{O}^{c}$. By repeated applications of Theorem 3.13, we can construct an $A \in\left[\operatorname{depth}_{B}(a), B\right]$ such that either $r_{k+1}[a, A] \subseteq \mathcal{O}$, or else $r_{k+1}[a, A] \subseteq \mathcal{O}^{c}$.

\section{RAMSEY-ClASSificAtion THEOREMS FOR $\mathcal{R}_{\alpha}, 2 \leq \alpha<\omega_{1}$}

This section contains the Ramsey-classification theorems for equivalence relations on fronts on the spaces $\mathcal{R}_{\alpha}, 2 \leq \alpha<\omega_{1}$. Recall Definitions 3.5, 3.6, and 3.8 of the various canonical equivalence relations. We provide new facts here, not in [5], necessitated by the fact that $\alpha$ may be an infinite, countable ordinal.

Fact 4.1. Let $n \leq m<\omega$ and $X \in \mathcal{R}_{\alpha}$, and suppose $\mathrm{R}$ is an equivalence relation on $\mathcal{R}_{\alpha}(n)$. Then there is an $S \in \mathfrak{S}(n, m)$ and a $Y \leq_{\alpha} X$ such that for each $y \in \mathcal{R}_{\alpha}(m) \mid Y, \mathrm{R} \uparrow\left(\mathcal{R}_{\alpha}(n) \mid y\right)$ is given by $\mathrm{E}_{S}$.

Proof. For each $S \in \mathfrak{S}(n, m)$, let

$$
\mathcal{X}_{S}=\left\{Y \leq_{\alpha} X: \mathrm{R} \uparrow\left(\mathcal{R}_{\alpha}(n) \mid Y(m)\right)=\mathrm{E}_{S}\right\} .
$$

Since $\mathfrak{S}(n, m)$ is finite, applying the Abstract Ellentuck Theorem and Theorem 3.12 for $\mathcal{R}_{\alpha}(n)$, we obtain an $S \in \mathfrak{S}(n, m)$ and a $Y \leq_{\alpha} X$ such that $[\emptyset, Y] \subseteq \mathcal{X}_{S}$.

It is useful to point out the following statement, which can be proved using $(\dagger)$ by a simple induction on $\alpha<\omega_{1}$ : For every $s \in \mathbb{S}_{\alpha}(n)$ which has domain $[\beta, \alpha]$ for some $\beta<\alpha$, there is an $n^{\prime}>n$ such that any embedding of $\mathbb{S}_{\alpha}(n)$ into $\mathbb{S}_{\alpha}\left(n^{\prime}\right)$ sends $s$ to the immediate successor of a splitting node in $\mathbb{S}_{\alpha}\left(n^{\prime}\right)$. By an embedding, we mean an injective, lexicographic order-preserving map $\iota$, such that if $s^{\prime}$ is an immediate predecessor of $s$, then $\iota\left(s^{\prime}\right)$ is an immediate predecessor of $\iota(s)$.

Fact 4.2. Let $n \leq m<m^{\prime}$ and $\mathrm{R}$ be an equivalence relation on $\mathcal{R}_{\alpha}(n)$. Suppose that $S \in \mathfrak{S}_{\alpha}(n, m), S^{\prime} \in \mathfrak{S}_{\alpha}\left(n, m^{\prime}\right)$, and $X \in \mathcal{R}_{\alpha}$ satisfies $\mathrm{R} \uparrow\left(\mathcal{R}_{\alpha}(n) \mid x\right)=\mathrm{E}_{S}$ for all $x \in \mathcal{R}_{\alpha}(m) \mid X$, and $\mathrm{R} \uparrow\left(\mathcal{R}_{\alpha}(n) \mid x\right)=\mathrm{E}_{S^{\prime}}$ for all $x \in \mathcal{R}_{\alpha}\left(m^{\prime}\right) \mid X$. Then $S^{\prime} \subseteq S$. Moreover, given any embedding $\iota: \mathbb{S}_{\alpha}(n) \rightarrow \mathbb{S}_{\alpha}(m)$, for every $s \in S$ such that $\iota(s)$ is an immediate successor of a splitting node in $\mathbb{S}_{\alpha}(m), s$ is also in $S^{\prime}$. 
Proof. Assuming the hypotheses, let $x \in \mathcal{R}_{\alpha}\left(m^{\prime}\right) \mid X$ and $z \in \mathcal{R}_{\alpha}(m) \mid x$. Then for all $y, y^{\prime} \in \mathcal{R}_{\alpha}(n) \mid z$, we have that also $y, y^{\prime} \in \mathcal{R}_{\alpha}(n) \mid x$. Thus, $y \mathrm{E}_{S} y^{\prime}$ implies $y \mathrm{R} y^{\prime}$, which in turn implies $y \mathrm{E}_{S^{\prime}} y^{\prime}$. Hence, $S^{\prime} \subseteq S$.

Suppose that there is an embedding $\iota: \mathbb{S}_{\alpha}(n) \rightarrow \mathbb{S}_{\alpha}(m)$ and an $s \in S \backslash S^{\prime}$ such that $\iota(s)$ is an immediate successor of some splitting node in $\mathbb{S}_{\alpha}(m)$. Then there are $x \in \mathcal{R}_{\alpha}\left(m^{\prime}\right) \mid X$ and $y, y^{\prime} \in \mathcal{R}_{\alpha}(n) \mid x$ such that $y \mathbb{E}_{S} y^{\prime}$ but $y \mathrm{E}_{S^{\prime}} y^{\prime}$, a contradiction.

The next theorem will be essential in the proof of the main theorem, Theorem 4.12. Lemma 4.4 is included, as the argument there will be useful elsewhere.

Theorem 4.3 (Canonization Theorem for Equivalence Relations on $\mathcal{R}_{\alpha}(n)$ ). Let $\mathrm{R}$ be an equivalence relation on $\mathcal{R}_{\alpha}(n)$ and let $X \in \mathcal{R}_{\alpha}(n)$. Then there is an $S \in \mathfrak{S}_{\alpha}(n)$ and $a Y \leq_{\alpha} X$ such that $\mathrm{R} \uparrow\left(\mathcal{R}_{\alpha}(n) \mid Y\right)$ is given by $\mathrm{E}_{S}$.

Proof. Assume the hypotheses. Recall the map $\pi_{n+1, n}^{\alpha}: \mathcal{R}_{\alpha}(n+1) \rightarrow \mathcal{R}_{\alpha}(n)$ from Definition 3.4. Let

$$
\mathcal{X}=\left\{X^{\prime} \leq_{\alpha} X: X^{\prime}(n) \mathrm{R} \pi_{n+1, n}^{\alpha}\left(X^{\prime}(n+1)\right)\right\} .
$$

This set $\mathcal{X}$ will tell us whether or not the lexicographically least node in $X^{\prime}(n)$ matters to the equivalence relation $\mathrm{R}$. By the Abstract Ellentuck Theorem, there is an $X^{\prime} \leq_{\alpha} X$ such that either $\left[\emptyset, X^{\prime}\right] \subseteq \mathcal{X}$ or $\left[\emptyset, X^{\prime}\right] \cap \mathcal{X}=\emptyset$. Possibly thinning again, letting $S_{\alpha}$ denote $\{(\alpha, n), \emptyset\} \in \mathfrak{S}_{\alpha}(n)$. We obtain a $Y \leq_{\alpha} X^{\prime}$ such that either

(i) for all $u, v \in \mathcal{R}_{\alpha}(n) \mid Y, u \mathrm{R} v$; or

(ii) for all $u, v \in \mathcal{R}_{\alpha}(n) \mid Y$, if $u \mathrm{R} v$, then $\pi_{S_{\alpha}}(u)=\pi_{S_{\alpha}}(v)$.

If case (i) holds, let $Z=Y$ and $S=\{\emptyset\}$. In this case, $u \mathrm{R} v$ for all $u, v \in \mathcal{R}_{\alpha}(n) \mid Z$. Otherwise, case (ii) holds.

Suppose $\alpha<\omega$. Then $\mathbb{S}_{\alpha}(n)$ is a finite tree, so $\mathfrak{S}_{\alpha}(n)$ is finite and consists of all finite subtrees of $\mathbb{S}_{\alpha}(n)$. Take $k>n$ large enough that $\mathfrak{S}_{\alpha}\left(n, k^{\prime}\right)=\mathfrak{S}_{\alpha}(n, k)$ for all $k^{\prime} \geq k$. For each $S \in \mathfrak{S}_{\alpha}(n) \backslash\{\emptyset\}$, define

$$
\mathcal{Y}_{S}=\left\{Y^{\prime} \leq_{\alpha} Y: \forall u, v \in \mathcal{R}_{\alpha}(n) \mid Y^{\prime}(2 k)\left(u \mathrm{R} v \text { iff } u \mathrm{E}_{S} v\right)\right\} .
$$

Let $\mathcal{Y}^{\prime}=[\emptyset, Y] \backslash \bigcup_{S \in \mathfrak{S}_{\alpha}(n, k)} \mathcal{Y}_{S}$. Then the $\mathcal{Y}_{S}, S \in \mathfrak{S}_{\alpha}(n) \backslash\{\emptyset\}$, along with $\mathcal{Y}^{\prime}$ form an open cover of $[\emptyset, Y]$. Since $\mathfrak{S}_{\alpha}(n)$ is finite, by the Abstract Ellentuck Theorem, there is a $Z \leq_{\alpha} Y$ such that either $[\emptyset, Z] \subseteq \mathcal{Y}_{S}$ for some $S \in \mathfrak{S}_{\alpha}(n) \backslash\{\emptyset\}$, or else $[\emptyset, Z] \subseteq \mathcal{Y}^{\prime}$. By Theorem 3.12 for $\mathcal{R}_{\alpha}$, it cannot be the case that $[\emptyset, Z] \subseteq \mathcal{Y}^{\prime}$. Since we are under the assumption that (ii) holds, there is some $S \in \mathfrak{S}_{\alpha}(n) \backslash\{\emptyset\}$ such that $\mathrm{R} \uparrow\left(\mathcal{R}_{\alpha}(n) \mid Z\right)$ is given by $\mathrm{E}_{S}$.

Now suppose that $\omega \leq \alpha<\omega_{1}$. Then $\mathbb{S}_{\alpha}(n)$ is not a tree, and $\mathfrak{S}_{\alpha}(n)$ is countably infinite.

Lemma 4.4. Suppose $\omega \leq \alpha<\omega_{1}$. Let $n<\omega, \mathrm{R}$ be an equivalence relation on $\mathcal{R}_{\alpha}(n)$, and $Y \in \mathcal{R}_{\alpha}$ such that (ii) holds; that is, for all $u, v \in \mathcal{R}_{\alpha}(n) \mid Y$, if $u \operatorname{R} v$, then $\pi_{S_{\alpha}}(u)=\pi_{S_{\alpha}}(v)$. Then there is a decreasing sequence, $Y=Y_{n} \geq_{\alpha}$ $Y_{n+1} \geq_{\alpha} \ldots$, and $S_{m} \in \mathfrak{S}_{\alpha}(n, m)$ for $m \geq n$ such that $S_{n} \supseteq S_{n+1} \supseteq \ldots$, and for each $m \geq n, \mathrm{R} \uparrow\left(\mathcal{R}_{\alpha}(n) \mid z\right)$ is given by $\mathrm{E}_{S_{m}}$ for each $z \in \mathcal{R}_{\alpha}(m) \mid Y_{m}$. Moreover, letting $Z=r_{n}^{\alpha}(Y) \cup \bigcup\left\{Y_{n}(n): n \geq m\right\}$ and $S=\bigcap\left\{S_{m}: m \geq n\right\}$, we have that $\mathrm{R} \uparrow\left(\mathcal{R}_{\alpha}(n) \mid Z\right)$ is given by $\mathrm{E}_{S}$. 
Proof. Assume the hypotheses. Let $S_{n}=\mathbb{S}_{\alpha}(n)$; this is the only member of $\mathfrak{S}_{\alpha}(n, n)$. By Fact 4.1, there is a $Y_{n+1} \leq_{\alpha} Y$ and an $S_{n+1} \in \mathfrak{S}(n, n+1)$ such that $\mathrm{R} \nmid \mathcal{R}_{\alpha}(n) \mid y$ is given by $\mathrm{E}_{S_{n+1}}$, for each $y \in \mathcal{R}_{\alpha}(n) \mid Y_{n+1}$. Given $Y_{m}, m>n$, by Fact 4.1, there is a $Y_{m+1} \leq_{\alpha} Y_{m}$ and an $S_{m+1} \in \mathfrak{S}(n, m+1)$ such that R $\left|\mathcal{R}_{\alpha}(n)\right| y$ is given by $\mathrm{E}_{S_{m+1}}$, for each $y \in \mathcal{R}_{\alpha}(n) \mid Y_{m+1}$. By Fact 4.2, $S_{m+1} \subseteq S_{m}$.

Let $Z=r_{n}^{\alpha}(Y) \cup \bigcup\left\{Y_{m}(m): m \geq n\right\}$, and let $S=\bigcap\left\{S_{m}: m \geq n\right\}$. Then $S$ is downward closed, so $S \in \mathfrak{S}_{\alpha}(n)$. Moreover, $S$ is nonempty, since the node $\{(\alpha, n)\} \in S_{m}$ for every $m \geq n$. We claim that $\mathrm{R}\left\lceil\mathcal{R}_{\alpha}(n) \mid Z\right.$ is given by $\mathrm{E}_{S}$. Let $u, v \in \mathcal{R}_{\alpha}(n) \mid Z$, and let $m, m^{\prime}$ be the integers such that $u \subseteq Z(m)$ and $v \subseteq Z\left(m^{\prime}\right)$. If $m \neq m^{\prime}$, then (ii) implies that $u \not R v$. Since $S$ is nonempty, also $u \mathbb{E}_{S} v$. Now suppose $m=m^{\prime}$. If $u \mathrm{R} v$, then $u \mathrm{E}_{S_{m}} v$, which implies $u \mathrm{E}_{S} v$, since $S \subseteq S_{m}$. If $u \not R v$, then $u \mathbb{E}_{S_{m}} v$. Let $s \in S_{m}$ be minimal in $S_{m}$ such that the copies of $s$ in $u$ and $v$ are different, under the isomorphisms of $\mathbb{S}_{\alpha}(n)$ into $u$ and $v$. Note that $s$ must be the immediate successor of some splitting node in $\mathbb{S}_{\alpha}(m)$. But then Fact 4.2 implies $s$ must be in $S_{k}$ for all $k \geq m$, which implies $s \in S$, a contradiction. Hence, also $u \mathrm{E}_{S} v$. Therefore, $u \mathrm{R} v$ iff $u \mathrm{E}_{S} v$.

By the lemma, the proof is complete.

The following Lemmas 4.5, 4.7 and 4.8 were proved as Lemmas 4.6, 4.9, and 4.10, respectively, in [5] for $\mathcal{R}_{1}$. As the proofs are identical for all the spaces $\mathcal{R}_{\alpha}$, $1 \leq \alpha<\omega_{1}$, we restate these lemmas without proof. In the following, $X /(a, b)$ denotes $X / a \cap X / b$.

Lemma 4.5. Suppose $1 \leq \alpha<\omega_{1}$.

(1) Suppose $P(\cdot, \cdot)$ is a property such that for each $a \in \mathcal{A R}^{\alpha}$ and each $X \in \mathcal{R}_{\alpha}$, there is a $Z \leq_{\alpha} X$ such that $P(a, Z)$ holds. Then for each $X \in \mathcal{R}_{\alpha}$, there is a $Y \leq_{\alpha} X$ such that for each $a \in \mathcal{A R}^{\alpha} \mid Y$ and each $Z \leq_{\alpha} Y, P(a, Z / a)$ holds.

(2) Suppose $P(\cdot, \cdot, \cdot)$ is a property such that for all $a, b \in \mathcal{A R}^{\alpha}$ and each $X \in \mathcal{R}_{\alpha}$, there is a $Z \leq_{\alpha} X$ such that $P(a, b, Z)$ holds. Then for each $X \in \mathcal{R}_{\alpha}$, there is a $Y \leq_{\alpha} X$ such that for all $a, b \in \mathcal{A R}^{\alpha} \mid Y$ and all $Z \leq_{\alpha} Y, P(a, b, Z /(a, b))$ holds.

Given a front $\mathcal{F}$ on $[\emptyset, A]$ for some $A \in \mathcal{R}_{\alpha}$ and $f: \mathcal{F} \rightarrow \mathbb{N}$, we adhere to the following convention: If we write $f(a)$ or $f(a \cup u)$, it is assumed that $a, a \cup u$ are in $\mathcal{F}$. Define

$$
\hat{\mathcal{F}}=\left\{r_{m}^{\alpha}(a): a \in \mathcal{F}, m \leq n<\omega, \text { where } a \in \mathcal{A R}_{n}^{\alpha}\right\} .
$$

Note that $\emptyset \in \hat{\mathcal{F}}$, since $\emptyset=r_{0}^{\alpha}(a)$ for any $a \in \mathcal{F}$. Recall that for $a \in \mathcal{A R}_{k}^{\alpha}$ and $m<n \leq k, a[m, n)$ denotes $\bigcup\{a(i): i \in[m, n)\}$. For any $X \leq_{\alpha} A$, define

$$
\operatorname{Ext}(X)=\left\{a[m, n): \exists m \leq n\left(a \in \mathcal{A R}_{n}^{\alpha}, \text { and } a[m, n) \subseteq X\right)\right\} .
$$

$\operatorname{Ext}(X)$ is the collection of all possible legal extensions into $X$. Note that $a[m, n) \subseteq$ $X$ iff $a[m, n) \leq_{\text {fin }}^{\alpha} X$. For any $a \in \mathcal{A R}^{\alpha}$, let $\operatorname{Ext}(X / a)$ denote the collection of those $y \in \operatorname{Ext}(X)$ such that $y \subseteq X / a$. Let $\operatorname{Ext}(X /(a, b))$ denote $\operatorname{Ext}(X / a) \cap \operatorname{Ext}(X / b)$. For $u \in \operatorname{Ext}(X)$, we write $v \in \operatorname{Ext}(u)$ to mean that $v \in \operatorname{Ext}(X)$ and $v \subseteq u$.

Definition 4.6. Fix $a, b \in \hat{\mathcal{F}}$ and $X \in \mathcal{R}_{\alpha}$. We say that $X$ separates $a$ and $b$ iff for all $x \in \operatorname{Ext}(X / a)$ and $y \in \operatorname{Ext}(X / b)$ such that $a \cup x$ and $b \cup y$ are in $\mathcal{F}$, $f(a \cup x) \neq f(b \cup y)$. We say that $X$ mixes $a$ and $b$ iff there is no $Y \leq_{\alpha} X$ which separates $a$ and $b$. $X$ decides for $a$ and $b$ iff either $X$ separates $a$ and $b$ or else $X$ mixes $a$ and $b$. 
We say that $X /(a, b)$ separates $a$ and $b$ iff for all $x, y \in \operatorname{Ext}(X /(a, b))$ such that $a \cup x$ and $b \cup y$ are in $\mathcal{F}, f(a \cup x) \neq f(b \cup y) . X /(a, b)$ mixes $a$ and $b$ iff there is no $Y \leq_{\alpha} X /(a, b)$ which separates $a$ and $b$. $X /(a, b)$ decides for $a$ and $b$ iff either $X /(a, b)$ separates $a$ and $b$, or else $X /(a, b)$ mixes $a$ and $b$.

The following facts are useful to note. $X$ mixes $a$ and $b$ iff $X /(a, b)$ mixes $a$ and $b$ iff for each $Y \leq_{\alpha} X$, there are $x, y \in \operatorname{Ext}(Y)$ such that $f(a \cup x)=f(b \cup y)$ iff for all $Y \leq_{\alpha} X, Y$ mixes $a$ and $b$. If $X$ separates $a$ and $b(X /(a, b)$ separates $a$ and $b)$, then for all $Y \leq_{\alpha} X$ (for all $Y \leq_{\alpha} X /(a, b)$ ), $Y$ separates $a$ and $b . X /(a, b)$ decides for $a$ and $b$ iff either for all $x, y \in \operatorname{Ext}(X /(a, b)), f(a \cup x) \neq f(b \cup y)$, or else there is no $Y \leq_{\alpha} X /(a, b)$ which has this property. Thus, $X /(a, b)$ mixes $a$ and $b$ iff $X$ mixes $a$ and $b$. However, if $X /(a, b)$ separates $a$ and $b$ it does not necessarily follow that $X$ separates $a$ and $b$.

Lemma 4.7 (Transitivity of mixing). For any $X \in \mathcal{R}_{\alpha}$ and any $a, b, c \in \hat{\mathcal{F}}$, if $X$ mixes $a$ and $b$ and $X$ mixes $b$ and $c$, then $X$ mixes $a$ and $c$.

Lemma 4.8. For each $X \in \mathcal{R}_{\alpha}$, there is a $Y \leq_{\alpha} X$ such that for each $a, b \leq_{\text {fin }}^{\alpha} Y$ in $\hat{\mathcal{F}}, Y /(a, b)$ decides for $a$ and $b$.

Definition 4.9. Let $\mathcal{F}$ be a front on $[\emptyset, X]$ for some $X \in \mathcal{R}_{\alpha}$, and let $\varphi$ be a function on $\mathcal{F}$.

(1) $\varphi$ is inner if $\varphi(a) \subseteq a$ for all $a \in \mathcal{F}$.

(2) $\varphi$ is Nash-Williams if $\varphi(a) \nsubseteq \varphi(b)$ for all $a \neq b \in \mathcal{F}$.

(3) $\varphi$ is Sperner if $\varphi(a) \nsubseteq \varphi(b)$ for all $a \neq b \in \mathcal{F}$

Definition 4.10. Let $X \in \mathcal{R}_{\alpha}, \mathcal{F}$ be a front on $[\emptyset, X]$, and $\mathrm{R}$ an equivalence relation on $\mathcal{F}$. We say that $\mathrm{R}$ is canonical if and only if there is an inner Nash-Williams function $\varphi$ on $\mathcal{F}$ such that

(1) for all $a, b \in \mathcal{F}, a \mathrm{R} b$ if and only if $\varphi(a)=\varphi(b)$; and

(2) $\varphi$ is maximal among all inner Nash-Williams functions satisfying (1); that is, for any other inner Nash-Williams function $\varphi^{\prime}$ on $\mathcal{F}$ satisfying (1), there is a $Y \leq_{\alpha} X$ such that $\varphi^{\prime}(a) \subseteq \varphi(a)$ for all $a \in \mathcal{F} \mid Y$.

Remark 4.11. As in [5], the map $\varphi$ constructed in the proof of Theorem 4.12 will in fact be Sperner. Moreover, this $\varphi$ is also the only such inner Nash-Williams map with the additional property $(*)$ that there is a $Z \leq_{\alpha} C$ such that for each $s \in \mathcal{F} \mid Z$ there is a $t \in \mathcal{F}$ such that $\varphi(s)=\varphi(t)=s \cap t$.

The following is part of the general induction scheme discussed in Section 3 .

Induction Hypothesis. Suppose that $2 \leq \alpha<\omega_{1}$; for all $1 \leq \beta<\alpha$, Theorems 4.12, 4.22 and 3.10 and Lemma 3.11 (in that order) hold for $\mathcal{R}_{\beta}$; and Theorems 3.12, 3.13, 3.14, and 4.3 hold for $\mathcal{R}_{\alpha}$.

Recall Remark 2.8, where for any front $\mathcal{F}$ on some $X \in \mathcal{R}_{\alpha}$, there is a $Y \leq_{\alpha} X$ such that $\mathcal{F} \mid Y$ is a barrier. Thus, the following main theorem yields the analogue of the Pudlak-Rödl Theorem.

Theorem 4.12. Suppose $A \in \mathcal{R}_{\alpha}, \mathcal{F}$ is a front on $[\emptyset, A]$ and $\mathrm{R}$ is an equivalence relation on $\mathcal{F}$. Then there is a $C \leq_{\alpha} A$ such that $\mathrm{R}$ is canonical on $\mathcal{F} \mid C$.

Proof. Let $A \in \mathcal{R}_{\alpha}$, let $\mathcal{F}$ be a given front on $[\emptyset, A]$, and let $\mathrm{R}$ be an equivalence relation on $\mathcal{F}$. Let $f: \mathcal{F} \rightarrow \mathbb{N}$ be any mapping which induces $\mathrm{R}$. By thinning if 
necessary, we may assume that $A$ satisfies Lemma 4.8, Let $(\hat{\mathcal{F}} \backslash \mathcal{F}) \mid X$ denote the collection of those $a \in \hat{\mathcal{F}} \backslash \mathcal{F}$ such that $a \leq_{\text {fin }}^{\alpha} X$.

Claim 4.13. There is a $B \leq_{\alpha} A$ such that for all $a \in(\hat{\mathcal{F}} \backslash \mathcal{F}) \mid B$, letting $n=|a|$, there is an $S_{a} \in \mathfrak{S}_{\alpha}(n)$ such that, letting $\mathrm{E}_{a}$ denote $\mathrm{E}_{S_{a}}$, for all $u, v \in \mathcal{R}_{\alpha}(n) \mid B / a$, $B$ mixes $a \cup u$ and $a \cup v$ if and only if $u \mathrm{E}_{a} v$.

Proof. For any $Z \leq_{\alpha} A$ and $a \in \mathcal{A R}^{\alpha} \mid A$, let $P(a, Z)$ denote the following statement: "If $a \in \hat{\mathcal{F}} \backslash \mathcal{F}$, then there is an $S_{a} \in \mathfrak{S}_{\alpha}(|a|)$ such that for all $u, v \in \mathcal{R}_{\alpha}(|a|) \mid Z / a, Z$ mixes $a \cup u$ and $a \cup v$ if and only if $u \mathrm{E}_{S_{a}} v$." We shall show that for each $X \leq_{\alpha} A$ and $a \in \mathcal{A R}^{\alpha} \mid A$, there is a $Z \leq_{\alpha} X$ for which $P(a, Z)$ holds. The claim then follows from Lemma 4.5

Let $X \leq_{\alpha} A$ and $a \in \hat{\mathcal{F}} \backslash \mathcal{F}$ be given, and let $n=|a|$. Let E denote the following equivalence relation of mixing on $\mathcal{R}_{\alpha}(n) \mid A / a$ : For all $u, v \in \mathcal{R}_{\alpha}(n) \mid A / a$,

$$
u \mathrm{E} v \Leftrightarrow A \text { mixes } a \cup u \text { and } a \cup v \text {. }
$$

By Theorem 4.3, there is an $S \in \mathfrak{S}_{\alpha}(n)$ and a $Y \leq_{\alpha} X$ such that $\mathrm{E} \uparrow\left(\mathcal{R}_{\alpha}(n) \mid Y\right)$ is given by $\mathrm{E}_{S}$. Take a $Z \leq_{\alpha} Y / a$ and let $S_{a}$ denote this $S$. Then $P(a, Z)$ holds.

Fix $B$ to be as in Claim 4.13, For $a \in(\hat{\mathcal{F}} \backslash \mathcal{F}) \mid B$ and $n=|a|$, let $S_{a}$ denote the member of $\mathfrak{S}_{\alpha}(n)$ such that $\mathrm{E}_{a}=\mathrm{E}_{S_{a}}$, and let $\mathrm{E}_{a}$ denote $\mathrm{E}_{S_{a}}$. We say that $a$ is $\mathrm{E}_{a}$-mixed by $B$, meaning that for all $u, v \in \mathcal{R}_{\alpha}(n) \mid B / a, B$ mixes $a \cup u$ and $a \cup v$ if and only if $u \mathrm{E}_{a} v$.

Definition 4.14. For $a \in \hat{\mathcal{F}}|B, n=| a \mid$, and $i<n$, define

$$
\varphi_{r_{i}^{\alpha}(a)}(a(i))=\pi_{S_{r_{i}^{\alpha}(a)}}(a(i)) .
$$

For $a \in \mathcal{F} \mid B$, define

$$
\varphi(a)=\bigcup_{i<|a|} \varphi_{r_{i}^{\alpha}(a)}(a(i)) .
$$

The proof of the following claim is exactly the same as the one given for Claim 4.17 in [5].

Claim 4.15. The following are true for all $X \leq_{\alpha} B$ and all $a, b \in \hat{\mathcal{F}} \mid B$ :

(A1) Suppose $a \notin \mathcal{F}$ and $n=|a|$. Then $X$ mixes $a \cup u$ and $t$ for at most one $\mathrm{E}_{a}$ equivalence class of $u$ 's in $\mathcal{R}_{\alpha}(n) \mid B / a$.

(A2) If $X /(a, b)$ separates $a$ and $b$, then $X /(a, b)$ separates $a \cup x$ and $b \cup y$ for all $x, y \in \operatorname{Ext}(X /(a, b))$ such that $a \cup x, b \cup y \in \hat{\mathcal{F}}$.

(A3) Suppose $a \notin \mathcal{F}$ and $n=|a|$. Then $S_{a}=\{\emptyset\}$ if and only if $X$ mixes $a$ and $a \cup u$ for all $u \in \mathcal{R}_{\alpha}(n) \mid B / a$.

(A4) If $a \sqsubset b$ and $\varphi(a)=\varphi(b)$, then $X$ mixes $a$ and $b$.

Claim 4.16. If $a, b \in(\hat{\mathcal{F}} \backslash \mathcal{F}) \mid B$ are mixed by $B$, then $S_{a}$ and $S_{b}$ are isomorphic. Moreover, there is a $C \leq_{\alpha} B$ such that for all $a, b \in(\hat{\mathcal{F}} \backslash \mathcal{F}) \mid C$, for all $u \in$ $\mathcal{R}_{\alpha}(|a|) \mid C /(a, b)$ and $v \in \mathcal{R}_{\alpha}(|b|) \mid C /(a, b), C$ mixes $a \cup u$ and $b \cup v$ if and only if $\varphi_{a}(u)=\varphi_{b}(v)$.

Proof. Suppose $a, b \in(\hat{\mathcal{F}} \backslash \mathcal{F}) \mid B$ are mixed by $B /(a, b)$, and let $X \leq_{\alpha} B$. By possibly thinning $X$, we may assume that $X \leq_{\alpha} B /(a, b)$. Let $i=|a|$ and $j=|b|$. 
Suppose that $S_{a}=\{\emptyset\}$ and $S_{b} \neq\{\emptyset\}$. By (A1), $B /(a, b)$ mixes $a$ and $b \cup v$ for at most one $\mathrm{E}_{a}$ equivalence class of $v^{\prime}$ 's in $\mathcal{R}_{\alpha}(j) \mid B / b$. Since $S_{b} \neq\{\emptyset\}$, there is a $Y \leq_{\alpha} X /(a, b)$ such that for each $v \in \mathcal{R}_{\alpha}(j) \mid Y, Y$ separates $a$ and $b \cup v$. Since $S_{a}=\{\emptyset\}$, it follows from (A4) that for all $u \in \mathcal{R}_{\alpha}(i) \mid Y, Y$ mixes $a$ and $a \cup u$. If there are $u \in \mathcal{R}_{\alpha}(i) \mid Y$ and $v \in \mathcal{R}_{\alpha}(j) \mid Y$ such that $Y$ mixes $a \cup u$ and $b \cup v$, then $Y$ mixes $a$ and $b \cup v$, by transitivity of mixing. This contradicts the fact that for each $v \in \mathcal{R}_{\alpha}(j) \mid Y, Y$ separates $a$ and $b \cup v$. Therefore, all extensions of $a$ and $b$ into $Y$ are separated. But then $a$ and $b$ are separated, a contradiction. Hence, $S_{b}$ must also be $\{\emptyset\}$. By a similar argument, we conclude that $S_{a}=\{\emptyset\}$ if and only if $S_{b}=\{\emptyset\}$. Hence, $\varphi_{a}(u)=\varphi_{b}(v)=\{\emptyset\}$ for all $u \in \mathcal{R}_{\alpha}(i) \mid B$ and $v \in \mathcal{R}_{\alpha}(j) \mid B$.

Suppose now that both $S_{a}$ and $S_{b}$ are not $\{\emptyset\}$. Let $X \leq_{\alpha} B /(a, b)$ and $m=$ $\max (i, j)+1$. Let

$$
\begin{aligned}
& \mathcal{Z}_{a}=\left\{Y \leq_{\alpha} X: B /(a, b) \text { separates } a \cup Y(i) \text { and } b \cup \pi_{j, m}^{\alpha}(Y(m))\right\}, \\
& \mathcal{Z}_{b}=\left\{Y \leq_{\alpha} X: B /(a, b) \text { separates } a \cup \pi_{i, m}^{\alpha}(Y(m)) \text { and } b \cup Y(j)\right\} .
\end{aligned}
$$

Applying the Abstract Ellentuck Theorem to the sets $\mathcal{Z}_{a}$ and $\mathcal{Z}_{b}$, we obtain an $X^{\prime} \leq_{\alpha} X$ such that $\left[0, X^{\prime}\right] \subseteq \mathcal{Z}_{a} \cap \mathcal{Z}_{b}$, since both $S_{a}$ and $S_{b}$ are not $\{\emptyset\}$. Thus, for all $u \in \mathcal{R}_{\alpha}(i) \mid X^{\prime}$ and $v \in \mathcal{R}_{\alpha}(j) \mid X^{\prime}, a \cup u$ and $b \cup v$ may be mixed by $B /(a, b)$ only if $u$ and $v$ are subtrees of the same $X^{\prime}(l)$ for some $l$.

For $l \in\{i, j\}$ and $k \geq m$, let $\mathfrak{I}_{\alpha}(l, k)$ denote the collection of all $S \subseteq \mathbb{S}_{\alpha}(k)$ such that $S \cong \mathbb{S}_{\alpha}(l)$. So $\mathfrak{I}_{\alpha}(l, k)$ consists of exactly those $S \in \mathfrak{S}_{\alpha}(k)$ such that $\pi_{S}: \mathcal{R}_{\alpha}(k) \rightarrow \mathcal{R}_{\alpha}(l)$. Note that each $\mathfrak{I}_{\alpha}(l, k)$ is finite. For each pair $S \in \mathfrak{I}_{\alpha}(i, k)$, $S^{\prime} \in \mathfrak{I}_{\alpha}(j, k)$, let

$$
\mathcal{X}_{S, S^{\prime}}=\left\{Y \leq_{\alpha} X^{\prime}: B \text { mixes } a \cup \pi_{S}(Y(k)) \text { and } b \cup \pi_{S^{\prime}}(Y(k))\right\} .
$$

Diagonalize over $k \geq m$ as follows. Let $Y_{m}=X^{\prime}$. Given $Y_{k}$, apply the Abstract Ellentuck Theorem to $\mathcal{X}_{S, S^{\prime}}$ for all pairs $S, S^{\prime}$ from $\mathfrak{I}_{\alpha}(i, k), S^{\prime} \in \mathfrak{I}_{\alpha}(j, k)$, respectively, to obtain a $Y_{k+1} \leq_{\alpha} Y_{k}$ which is homogeneous for $\mathcal{X}_{S, S^{\prime}}$, for each such pair. Define

$$
Y=r_{m}^{\alpha}\left(Y_{m}\right) \cup \bigcup\left\{Y_{k+1}(k): k \geq m\right\}
$$

Then $Y$ is homogeneous for $\mathcal{X}_{S, S^{\prime}}$ for all $k \geq m$ and all pairs $S \in \mathfrak{I}_{\alpha}(i, k), S^{\prime} \in$ $\mathfrak{I}_{\alpha}(j, k)$.

Subclaim. There is a $Z \leq_{\alpha} Y$ such that for each $k \geq m$, each pair $S \in \mathfrak{I}_{\alpha}(i, k)$, $S^{\prime} \in \mathfrak{I}_{\alpha}(j, k)$, and each $Z^{\prime} \leq_{\alpha} Z$, if $\varphi_{s}\left(\pi_{S}\left(Z^{\prime}(k)\right)\right) \neq \varphi_{t}\left(\pi_{S^{\prime}}\left(Z^{\prime}(k)\right)\right)$, then $\left[\emptyset, Z^{\prime}\right] \cap$ $\mathcal{X}_{S, S^{\prime}}=\emptyset$.

Suppose not. Then in particular for $Y$, there are $k, S \in \mathfrak{I}_{\alpha}(i, k), S^{\prime} \in \mathfrak{I}_{\alpha}(j, k)$, and $Z \leq_{\alpha} Y$ such that $\varphi_{s}\left(\pi_{S}(Z(k))\right) \neq \varphi_{t}\left(\pi_{S^{\prime}}(Z(k))\right)$, but $[0, Z] \cap \mathcal{X}_{S, S^{\prime}} \neq \emptyset$. Since $Y$ is already homogeneous for $\mathcal{X}_{S, S^{\prime}}$, it must be the case that $[0, Y] \subseteq \mathcal{X}_{S, S^{\prime}}$; hence, $[0, Z] \subseteq \mathcal{X}_{S, S^{\prime}}$. Furthermore, $\varphi_{s}\left(\pi_{S}(Z(k))\right) \neq \varphi_{t}\left(\pi_{S^{\prime}}(Z(k))\right)$ implies that $\varphi_{s}\left(\pi_{S}\left(Z^{\prime}(k)\right)\right) \neq \varphi_{t}\left(\pi_{S^{\prime}}\left(Z^{\prime}(k)\right)\right)$ for all $Z^{\prime} \leq_{\alpha} Z$, since $\varphi_{s}, \pi_{S}, \varphi_{t}$, and $\pi_{S^{\prime}}$ are projection maps.

We claim that $\pi_{S_{a}}(S)=\pi_{S_{b}}\left(S^{\prime}\right)$. Suppose there is some $s \in \pi_{S_{a}}(S) \backslash \pi_{S_{b}}\left(S^{\prime}\right)$. Take $w, w^{\prime} \in \mathcal{R}_{\alpha}(k) \mid Z\left(k^{\prime}\right)$ for some $k^{\prime}$ large enough such that $w$ and $w^{\prime}$ differ exactly on their elements in the place $s$ and all extensions of $s$. Let $u=\pi_{S_{a}} \circ \pi_{S}(w)$, $u^{\prime}=\pi_{S_{a}} \circ \pi_{S}\left(w^{\prime}\right), v=\pi_{S_{b}} \circ \pi_{S^{\prime}}(w)$, and $v^{\prime}=\pi_{S_{b}} \circ \pi_{S^{\prime}}\left(w^{\prime}\right)$. Then $u \mathbb{E}_{a} u^{\prime}$ but 
$v \mathrm{E}_{b} v^{\prime}$. Since $[\emptyset, Z] \subseteq \mathcal{X}_{S, S^{\prime}}, B /(a, b)$ mixes $a \cup u$ and $b \cup v$, and $B /(a, b)$ mixes $a \cup u^{\prime}$ and $b \cup v^{\prime} . B /(a, b)$ mixes $b \cup v$ and $b \cup v^{\prime}$, since $v \mathrm{E}_{b} v^{\prime}$. Hence, by transitivity of mixing, $B /(a, b)$ mixes $a \cup u$ and $a \cup u^{\prime}$, contradicting that $u \mathbb{E}_{a} u^{\prime}$. Likewise, we obtain a contradiction if there is some $s \in \pi_{S_{b}}\left(S^{\prime}\right) \backslash \pi_{S_{a}}(S)$. Therefore, the Subclaim holds.

By the Subclaim, the following holds. There is a $Z \leq_{\alpha} Y$ such that for all $u \in \mathcal{R}_{\alpha}(i) \mid Z$ and $v \in \mathcal{R}_{\alpha}(j) \mid Z$, if $a \cup u$ and $b \cup v$ are mixed by $B /(a, b)$, then $\varphi_{a}(u)=\varphi_{b}(v)$. It follows that $S_{a}$ and $S_{b}$ must be isomorphic. Thus, we have shown that there is a $Z \leq_{\alpha} X$ such that for all $u \in \mathcal{R}_{\alpha}(i) \mid Z$ and $v \in \mathcal{R}_{\alpha}(j) \mid Z$, if $B /(a, b)$ mixes $a \cup u$ and $b \cup v$, then $\varphi_{a}(u)=\varphi_{b}(v)$.

It remains to show that for all $u \in \mathcal{R}_{\alpha}(i) \mid Z$ and $v \in \mathcal{R}_{\alpha}(j) \mid Z$, if $\varphi_{a}(u)=\varphi_{b}(v)$, then $Z$ mixes $a \cup u$ and $b \cup v$. Let $k \geq m$ and let $S \in \mathfrak{I}_{\alpha}(i, k), S^{\prime} \in \mathfrak{I}_{\alpha}(j, k)$ be any pair such that for all $w \in \mathcal{R}_{\alpha}(k) \mid Z, \varphi_{a}\left(\pi_{S}(w)\right)=\varphi_{b}\left(\pi_{S^{\prime}}(w)\right)$. We will show that $[\emptyset, Z] \subseteq \mathcal{X}_{S, S^{\prime}}$

Assume towards a contradiction that $[\emptyset, Z] \cap \mathcal{X}_{S, S^{\prime}}=\emptyset$. Then for all $w \in$ $\mathcal{R}_{\alpha}(k) \mid Z, Z$ separates $a \cup \pi_{S}(w)$ and $b \cup \pi_{S^{\prime}}(w)$. First, let $T \in \mathfrak{I}_{\alpha}(i, k), T^{\prime} \in \mathfrak{I}_{\alpha}(j, k)$ be any pair such that $\varphi_{a}\left(\pi_{T}(x)\right)=\varphi_{b}\left(\pi_{T^{\prime}}(x)\right)$ for any (all) $x \in \mathcal{R}_{\alpha}(k) \mid Z$. Then there are $x, y \in \mathcal{R}_{\alpha}(k) \mid Z$ such that $\pi_{S}(x) \mathrm{E}_{a} \pi_{T}(y)$ and $\pi_{S^{\prime}}(x) \mathrm{E}_{b} \pi_{T^{\prime}}(y) . Z$ mixes $a \cup \pi_{S}(x)$ and $a \cup \pi_{T}(y)$, and $Z$ mixes $b \cup \pi_{S^{\prime}}(x)$ and $b \cup \pi_{T^{\prime}}(y)$. Thus, $Z$ must separate $a \cup \pi_{T}(w)$ and $b \cup \pi_{T^{\prime}}(w)$ for all $w \in \mathcal{R}_{\alpha}(k) \mid Z$. Second, let $T, T^{\prime}$ be any pair such that $\varphi_{a}\left(\pi_{T}(x)\right) \neq \varphi_{b}\left(\pi_{T^{\prime}}(x)\right)$. Then $Z$ separates $a \cup \pi_{T}(x)$ and $b \cup \pi_{T^{\prime}}(x)$. Thinning, we obtain a $Z^{\prime} \leq_{\alpha} Z / r_{k}^{\alpha}(Z)$ which separates $a$ and $b$, a contradiction. Therefore, $[\emptyset, Z] \subseteq \mathcal{X}_{S, S^{\prime}}$, and thus $Z$ mixes $a \cup \pi_{S}(W(k))$ and $b \cup \pi_{S^{\prime}}(W(k))$ for all $W \leq_{\alpha} Z$.

Hence, for all such pairs $S, S^{\prime}$, we have that $\varphi_{a}\left(\pi_{S}(w)\right)=\varphi_{b}\left(\pi_{S^{\prime}}(w)\right)$ if and only if $[\emptyset, Z] \subseteq \mathcal{X}_{S, S^{\prime}}$. Thus, for all $u \in \mathcal{R}_{\alpha} \mid Z$ and $v \in \mathcal{R}_{\alpha} \mid Z, Z$ mixes $a \cup u$ and $b \cup v$ if and only if $\varphi_{a}(u)=\varphi_{b}(v)$.

Finally, we have shown that for all $a, b \in(\hat{\mathcal{F}} \backslash \mathcal{F}) \mid B$ and each $X \leq_{\alpha} B$, there is a $Z \leq_{\alpha} X$ such that for all $u \in \mathcal{R}_{\alpha}(i) \mid Z$ and $v \in \mathcal{R}_{\alpha}(j) \mid Z, Z$ mixes $a \cup u$ and $b \cup v$ if and only if $\varphi_{a}(u)=\varphi_{b}(v)$. By Lemma 4.5, there is a $C \leq_{\alpha} B$ for which the claim holds.

The proofs of the next three claims are the same as the proofs of Claims 4.19, 4.20 and 4.21 in [5].

Claim 4.17. For all $a, b \in \hat{\mathcal{F}} \mid C$, if $\varphi(a)=\varphi(b)$, then $a$ and $b$ are mixed by $C$. Hence, for all $a, b \in \mathcal{F} \mid C$, if $\varphi(a)=\varphi(b)$, then $f(a)=f(b)$.

Claim 4.18. For all $a, b \in \mathcal{F} \mid C, \varphi(a) \not \subset \varphi(b)$.

Claim 4.19. For all $a, b \in \mathcal{F} \mid C$, if $f(a)=f(b)$, then $\varphi(a)=\varphi(b)$.

It remains to show that $\varphi$ witnesses that $\mathrm{R}$ is canonical. By definition, $\varphi$ is inner, and by Claim 4.18, $\varphi$ is Nash-Williams. By Claims 4.17 and 4.19, we have that for each $a, b \in \mathcal{F} \mid C, a \mathrm{R} b$ if and only if $\varphi(a)=\varphi(b)$. Thus, it only remains to show that $\varphi$ is maximal among all inner Nash-Williams maps $\varphi^{\prime}$ on $\mathcal{F} \mid C$ which also represent the equivalence relation $\mathrm{R}$. Toward this end, we prove the following lemma. 
Lemma 4.20. Suppose $X \leq_{\alpha} C$ and $\varphi^{\prime}$ is an inner function on $\mathcal{F} \mid X$ which represents $\mathrm{R}$. Then there is a $Y \leq_{\alpha} X$ such that for each $a \in \mathcal{F} \mid Y$, for each $i<|a|$, there is an $S_{r_{i}^{\alpha}(a)}^{\prime} \in \mathfrak{S}_{\alpha}(i)$ such that $S_{r_{i}^{\alpha}(a)}^{\prime} \subseteq S_{r_{i}^{\alpha}(a)}$ and the following hold:

(1) For each $b \in \mathcal{F} \mid Y$ for which $b \sqsupset r_{i}^{\alpha}(a), \varphi^{\prime}(b) \cap b(i)=\pi_{S_{r_{i}^{\alpha}(a)}^{\prime}}(b(i))$.

(2) $\varphi^{\prime}(a)=\bigcup\left\{\pi_{S_{r_{i}^{\alpha}(a)}^{\prime}}(a(i)): i<|a|\right\} \subseteq \varphi(a)$.

Proof. Let $X \leq{ }_{\alpha} C$ and $\varphi^{\prime}$ satisfy the hypotheses. Fix any $a \in(\hat{\mathcal{F}} \backslash \mathcal{F}) \mid C, i<$ $|a|$, and $X^{\prime} \leq_{\alpha} X / a$. For each $k \geq i$ and $S \in \mathfrak{S}_{\alpha}(i, k)$, let $\mathcal{X}_{S}=\left\{Y \leq_{\alpha} X^{\prime}\right.$ : $\left.\varphi^{\prime}(a \cup Y[i, j)) \cap Y(i)=\pi_{S}(Y(i))\right\}$, where $j$ is such that $a \cup Y[i, j) \in \mathcal{F}$. Since $\varphi^{\prime}$ is inner, following the argument in Lemma 4.4 we construct an $X^{\prime \prime} \leq_{\alpha} X^{\prime}$ such that the following holds: There is an $S_{r_{i}^{\alpha}(a)}^{\prime} \in \mathfrak{S}_{\alpha}(i)$ such that for each $b \in \mathcal{F}$ extending $r_{i}^{\alpha}(a)$ with $b / r_{i}^{\alpha}(a) \in \operatorname{Ext}\left(X^{\prime \prime}\right), \varphi^{\prime}(b) \cap b(i)=\pi_{S_{r_{i}^{\alpha}(a)}^{\prime}}(b(i))$. By Lemma 4.5, there is a $Y \leq_{\alpha} X$ such that for each $a \in \mathcal{F} \mid Y$ and each $i^{2}<|a|$, there is an $S_{r_{i}^{\alpha}(a)}^{\prime}$ satisfying (1). Thus, for each $a \in \mathcal{F} \mid Y$,

$$
\varphi^{\prime}(t)=\bigcup\left\{\pi_{S_{r_{i}^{\alpha}(a)}^{\prime}}(a(i)): i<|a|\right\} .
$$

Note that each $S_{r_{i}^{\alpha}(a)}^{\prime}$ must be contained within the $S_{r_{i}^{\alpha}(a)}$ for the $\varphi$ already attained associated with the $\mathrm{E}_{r_{i}^{\alpha}(a)}$-mixing of immediate extensions of $r_{i}^{\alpha}(a)$. Otherwise, there would be $u, v \in \mathcal{R}_{\alpha}(i) \mid Y / r_{i}^{\alpha}(a)$ such that $r_{i}^{\alpha}(a) \cup u$ and $r_{i}^{\alpha}(a) \cup v$ are mixed, yet all extensions of them have different $\varphi^{\prime}$ values, which would contradict that $\varphi^{\prime}$ induces the same equivalence relation as $f$. Thus, for each $a \in \mathcal{F} \mid Y$, $\varphi^{\prime}(a) \subseteq \varphi(a)$.

By Lemma 4.20, $\mathrm{R}$ is canonical on $\mathcal{F} \mid C$, which finishes the proof of the theorem.

Remark 4.21. The map $\varphi$ from Theorem 4.12 has the following property. One can thin to a $Z$ such that

$(*)$ for each $s \in \mathcal{F} \mid Z$, there is a $t \in \mathcal{F}$ such that $\varphi(s)=\varphi(t)=s \cap t$.

This is not the case for any smaller inner map $\varphi^{\prime}$, by Lemma 4.20. For suppose $\varphi^{\prime}$ is an inner map representing $\mathrm{R}, \varphi^{\prime}$ satisfies the conclusions of Lemma 4.20 on $\mathcal{F} \mid Y$, and there is an $s \in \mathcal{F} \mid Y$ for which $\varphi^{\prime}(s) \subsetneq \varphi(s)$. Then there is some $i<|s|$ for which $S_{r_{i}^{\alpha}(s)}^{\prime} \subsetneq S_{r_{i}^{\alpha}(s)}$. This implies that $\varphi^{\prime}(t) \subsetneq \varphi(t)$ for every $t \in \mathcal{F} \mid Y$ such that $t \sqsupset r_{i}(s)$. Recall that $\varphi^{\prime}(t)=\varphi^{\prime}(s)$ if and only if $\varphi(t)=\varphi(s)$; and in this case, $\varphi(t) \cap \varphi(s) \subseteq t \cap s$. It follows that for any $t$ for which $\varphi^{\prime}(t)=\varphi^{\prime}(s), \varphi^{\prime}(t) \cap \varphi^{\prime}(s)$ will always be a proper subset of $t \cap s$. Thus, $\varphi$ is the minimal inner map for which property $(*)$ holds.

As shown in [5] for $\mathcal{R}_{1}$, this is the best possible: there are examples of fronts on which there are inner maps $\varphi^{\prime}$ such that $\varphi^{\prime}(a) \subsetneq \varphi(a)$ for all $a \in \mathcal{F} \mid C$.

Recall Definition 3.8. For $n<\omega$ and $X \in \mathcal{R}_{\alpha}$, an equivalence relation $\mathrm{R}$ on the front $\mathcal{A R}_{n}^{\alpha} \mid X$ is canonical iff for each $i<n$ there is an $S(i) \in \mathfrak{S}_{\alpha}(i)$ such that

$$
\forall a, b \in \mathcal{A R}_{n}^{\alpha} \mid X, a \mathrm{R} b \Leftrightarrow \forall i<n\left(\pi_{S(i)}(a(i))=\pi_{S(i)}(b(i))\right) .
$$

Note that if $n=0$, then $\mathcal{A R}_{0}^{\alpha}=\{\emptyset\}$, and every equivalence relation on $\{\emptyset\}$ is trivially canonical. 
Theorem 4.22 (Canonization Theorem for $\mathcal{A R}_{n}^{\alpha}$ ). Let $1 \leq n<\omega$. Given any $A \in \mathcal{R}_{\alpha}$ and any equivalence relation $\mathrm{R}$ on $\mathcal{A R}_{n}^{\alpha} \mid A$, there is a $D \leq_{\alpha} A$ such that $\mathrm{R}$ is canonical on $\mathcal{A R}_{n}^{\alpha} \mid D$.

Proof. Let $C \leq_{\alpha} A$ be obtained from Theorem 4.12. Then for each $a \in \mathcal{A R}_{n}^{\alpha} \mid C$, there is a sequence $\left\langle S_{r_{i}^{\alpha}(a)}: i<n\right\rangle$, where each $S_{r_{i}^{\alpha}(a)} \in \mathfrak{S}_{\alpha}(i)$, satisfying the following: For all $a, b \in \mathcal{A R}_{n}^{\alpha} \mid C$,

$$
a \mathrm{R} b \Leftrightarrow \bigcup_{i<n} \pi_{S_{r_{i}^{\alpha}(a)}}(a(i))=\bigcup_{i<n} \pi_{S_{r_{i}^{\alpha}(b)}}(b(i)) .
$$

We shall obtain a $D \leq{ }_{\alpha} C$ such that for all $a, b \in \mathcal{A R}_{n}^{\alpha} \mid D$ and all $i<n, S_{r_{i}^{\alpha}(a)}=$ $S_{r_{i}^{\alpha}(b)}$.

By the proof of Theorem 4.12, for all $a, b \in \mathcal{A R}_{n}^{\alpha} \mid C, S_{r_{0}^{\alpha}(a)}=S_{r_{0}^{\alpha}(b)}$. Let $X_{0}=C$ and $S(0)=S_{r_{0}^{\alpha}(a)}$ for any (all) $a \in \mathcal{A R}_{n}^{\alpha} \mid C$. Suppose $j \leq n-1$ and for all $i<j$, $X_{i}$, and $S(i)$ such that $\left[\emptyset, X_{i}\right] \subseteq \mathcal{X}_{S(i)}$, where $\mathcal{X}_{S(i)}=\left\{X \leq_{\alpha} C: S_{r_{i}^{\alpha}(X)}=S(i)\right\}$. For each $k \geq j$ and each $S \in \mathfrak{S}_{\alpha}(j, k)$, define

$$
\mathcal{X}_{S}(j, k)=\left\{X \leq_{\alpha} C: \pi_{S_{r_{j}^{\alpha}}(X)}\left\lceil\mathcal{R}_{\alpha}(j) \mid X(k)=\pi_{S}\left\lceil\mathcal{R}_{\alpha}(j) \mid X(k)\right\}\right.\right.
$$

These finitely many open sets, $\mathcal{X}_{S}(j, k), S \in \mathfrak{S}_{\alpha}(j, k)$, cover $[\emptyset, C]$. Diagonalizing over all $k \geq j$ as in the proof of Lemma 4.4, there is some $S(j) \in \mathfrak{S}_{\alpha}(j)$ and some $X_{j} \leq_{\alpha} X_{j-1}$ such that $\left[\emptyset, X_{j}\right] \subseteq \mathcal{X}_{S(j)}$, where $\mathcal{X}_{S(j)}=\left\{X \leq_{\alpha} C: S_{r_{j}^{\alpha}(X)}=S(j)\right\}$.

Let $D=X_{n-1}$. Then for all $a, b \in \mathcal{A R}_{n}^{\alpha} \mid D$,

$$
\begin{aligned}
a \mathrm{R} b & \Leftrightarrow \varphi(a)=\varphi(b) \\
& \Leftrightarrow \forall i<n, \pi_{S_{r_{i}^{\alpha}(a)}}(a(i))=\pi_{S_{r_{i}^{\alpha}(b)}}(b(i)) \\
& \Leftrightarrow \forall i<n, \pi_{S_{(i)}}(a(i))=\pi_{S(i)}(b(i)) \\
& \Leftrightarrow \forall i<n, a(i) \mathrm{E}_{S(i)} b(i) .
\end{aligned}
$$

Thus, the equivalence relation $\mathrm{R}$ is canonical on $\mathcal{A R}_{n}^{\alpha} \mid D$.

\section{The structure of the Rudin-Keisler Classes inside the Tukey types OF UltRAFILTERS TUKEY REDUCiBle TO $\mathcal{U}_{\alpha}$}

In this section, for each $\alpha<\omega_{1}$, we classify the Rudin-Keisler classes within the Tukey type of any ultrafilter Tukey reducible to $\mathcal{U}_{\alpha}$, the ultrafilter corresponding to the space $\mathcal{R}_{\alpha}$. As a corollary, we show that the Tukey types of ultrafilters Tukey reducible to $\mathcal{U}_{\alpha}$ form a decreasing chain of rapid p-points $\left\langle\mathcal{Y}_{\beta}^{\alpha}: \beta \leq \alpha\right\rangle$ of order type $\alpha+1$, where $\mathcal{Y}_{0}^{\alpha} \cong \mathcal{U}_{\alpha}$ and $\mathcal{Y}_{\alpha}^{\alpha}$ is Ramsey. We show that the isomorphism classes of the p-points in $\left[\mathcal{Y}_{\beta}^{\alpha}\right]_{T}$ are exactly those given by projections $\pi_{S}\left(\mathcal{U}_{\alpha}\left\lceil\mathcal{R}_{\alpha}(n)\right), n<\omega\right.$, such that $\mathbb{S}_{\alpha}(0) \uparrow[\beta, \alpha]$ embeds into $S$ but $\mathbb{S}_{\alpha}(0) \uparrow[\gamma, \alpha]$ does not embed into $S$ for any $\gamma<\beta$. Equivalently, the isomorphism classes of p-points in $\left[\mathcal{Y}_{\beta}^{\alpha}\right]_{T}$ are computed by the canonical equivalence relations on $\mathcal{R}_{\alpha}(n), n<\omega$. The isomorphism classes of ultrafilters within $\left[\mathcal{Y}_{\beta}^{\alpha}\right]_{T}$ are given by tree ultrafilters, where each node branches into some p-point of the sort $\pi_{S}\left(\mathcal{U}_{\alpha} \uparrow \mathcal{R}_{\alpha}(n)\right)$. A fuller discussion is presented towards the end of this section.

Recall that every topological Ramsey space has its own notion of Ramsey and selective ultrafilters (see [11]). Recall the following definitions from [5]. 
Definition $5.1([5],[1])$. Let $(\mathcal{R}, \leq, r)$ be any topological Ramsey space.

(1) We say that a subset $\mathcal{C} \subseteq \mathcal{R}$ satisfies the Abstract Nash-Williams Theorem if and only if for each family $\mathcal{G} \subseteq \mathcal{A R}$ and partition $\mathcal{G}=\mathcal{G}_{0} \cup \mathcal{G}_{1}$, there is a $C \in \mathcal{C}$ and an $i \in 2$ such that $\mathcal{G}_{i} \mid C=\emptyset$.

(2) We say that an ultrafilter $\mathcal{U}$ is Ramsey for $\mathcal{R}$ if and only if $\mathcal{U}$ is generated by a subset $\mathcal{C} \subseteq \mathcal{R}$ which satisfies the Abstract Nash-Williams Theorem.

(3) An ultrafilter generated by a set $\mathcal{C} \subseteq \mathcal{R}$ is selective for $\mathcal{R}$ if and only if for each decreasing sequence $X_{0} \geq X_{1} \geq \ldots$ of members of $\mathcal{C}$, there is another $X \in \mathcal{C}$ such that for each $n<\omega, X \leq X_{n} / r_{n}\left(X_{n}\right)$.

(4) We say that an ultrafilter $\mathcal{U}$ is canonical for fronts on $\mathcal{R}$ if and only if for any front $\mathcal{F}$ on $\mathcal{R}$ and any equivalence relation $\mathrm{R}$ on $\mathcal{F}$, there is a $U \in \mathcal{U} \cap \mathcal{R}$ such that $\mathrm{R}$ is canonical on $\mathcal{F} \mid U$.

We fix the following conventions and notation for the rest of this section.

Notation. For each $\alpha<\omega_{1}$, [ $\mathbb{T}_{\alpha}$ ] denotes the collection of all maximal nodes in $\mathbb{T}_{\alpha}$. Let $\mathcal{U}_{\alpha}$ denote any ultrafilter on base set $\left[\mathbb{T}_{\alpha}\right]$ such that $\left\{X \in \mathcal{R}_{\alpha}:[X] \in \mathcal{U}_{\alpha}\right\}$ is Ramsey for $\mathcal{R}_{\alpha}$ and canonical for fronts on $\mathcal{R}_{\alpha}$. Let $\mathcal{C}_{\alpha}$ denote $\left\{X \in \mathcal{R}_{\alpha}:[X] \in\right.$ $\left.\mathcal{U}_{\alpha}\right\}$. Note that $\left\{[X]: X \in \mathcal{C}_{\alpha}\right\}$ is cofinal in $\mathcal{U}_{\alpha}$. Moreover, since $X \rightarrow[X]$ is a bijection between $\mathcal{R}_{\alpha}$ and $\left\{[X]: X \in \mathcal{R}_{\alpha}\right\}$, there is no ambiguity going between these two sets; henceforth, we shall use them interchangeably.

We shall say that $\mathcal{F} \subseteq \mathcal{A} \mathcal{R}^{\alpha}$ is a front on a set $\mathcal{C} \subseteq \mathcal{R}_{\alpha}$ if $\mathcal{F}$ is Nash-Williams, and for each $X \in \mathcal{C}$, there is an $a \in \mathcal{F}$ such that $a \sqsubset X$. For any front $\mathcal{F}$ on $\mathcal{C}_{\alpha}$ and any $X \in \mathcal{C}_{\alpha}$, recall that $\mathcal{F} \mid X$ denotes $\left\{a \in \mathcal{F}: a \leq_{\text {fin }}^{\alpha} X\right\}$. Let

$$
\mathcal{C}_{\alpha}\left\lceil\mathcal{F}=\left\{\mathcal{F} \mid X: X \in \mathcal{C}_{\alpha}\right\} .\right.
$$

For each $\alpha<\omega_{1}$, ultrafilters $\mathcal{U}_{\alpha}$ which are Ramsey for $\mathcal{R}_{\alpha}$ and canonical for fronts on $\mathcal{R}_{\alpha}$ exist, assuming $\mathrm{CH}$ or MA, or forcing with $\left(\mathcal{R}_{\alpha}, \leq_{\alpha}^{*}\right)$. In fact, every generic ultrafilter forced by $\left(\mathcal{R}_{\alpha}, \leq_{\alpha}^{*}\right)$ is Ramsey for $\mathcal{R}_{\alpha}$ and canonical for fronts on $\mathcal{R}_{\alpha}$. Since $\mathcal{R}_{\alpha}$ is isomorphic to a dense subset of Laflamme's forcing $\mathbb{P}_{\alpha}$ in [10], any ultrafilter forced by $\left(\mathcal{R}_{\alpha}, \leq_{\alpha}^{*}\right)$ is isomorphic to an ultrafilter forced by $\left(\mathbb{P}_{\alpha}, \leq_{\mathbb{P}_{\alpha}}^{*}\right)$. Note that $\mathcal{C}_{\alpha}$ is cofinal in $\mathcal{U}_{\alpha}$.

We say that $\mathcal{B} \subseteq \mathcal{C}_{\alpha}$ generates $\mathcal{U}_{\alpha}$ if $\left\{[X]: X \in \mathcal{B}\right.$ is cofinal in $\mathcal{U}_{\alpha}$.

Fact 5.2. For each $\alpha<\omega_{1}$, the following hold:

(1) If $\mathcal{B} \subseteq \mathcal{C}_{\alpha}$ generates $\mathcal{U}_{\alpha}$, then for each front $\mathcal{F}$ on $\mathcal{B}$ and each $\mathcal{G} \subseteq \mathcal{F}$, there is a $U \in \mathcal{B}$ such that either $\mathcal{F} \mid U \subseteq \mathcal{G}$, or else $\mathcal{F} \mid U \cap \mathcal{G}=\emptyset$.

(2) Any ultrafilter Ramsey for $\mathcal{R}_{\alpha}$ is also selective for $\mathcal{R}_{\alpha}$.

(1) follows immediately from the definition of $\mathcal{U}_{\alpha} ;(2)$ is a consequence of Lemma 3.8 in 11 .

Given a front $\mathcal{F}$ on $\mathcal{C}_{\alpha}$, we let $\mathcal{U}_{\alpha} \uparrow \mathcal{F}$ denote the ultrafilter on base set $\mathcal{F}$ generated by the sets $\mathcal{F} \mid X, X \in \mathcal{C}_{\alpha}$. The proofs of Facts 5.3 and 5.4 and Proposition 5.5 are the same as the proofs of Facts 5.3 and 5.4 and Proposition 5.5 in [5].

Fact 5.3. Let $\alpha<\omega_{1}, \mathcal{B}$ be any cofinal subset of $\mathcal{C}_{\alpha}$, and $\mathcal{F} \subseteq \mathcal{A} \mathcal{R}^{\alpha}$ be any front on $\mathcal{C}_{\alpha}$. Then $\mathcal{B}\left\lceil\mathcal{F}\right.$ generates the ultrafilter $\mathcal{U}_{\alpha} \uparrow \mathcal{F}$ on the base set $\mathcal{F}$.

Fact 5.4. Suppose $\mathcal{U}$ and $\mathcal{V}$ are proper ultrafilters on the same countable base set, and for each $V \in \mathcal{V}$ there is a $U \in \mathcal{U}$ such that $U \subseteq V$. Then $\mathcal{U}=\mathcal{V}$. 
Recall that by Theorem 20 in [4, every Tukey reduction from a p-point to another ultrafilter is witnessed by a continuous cofinal map. By arguments from [5], the following holds.

Proposition 5.5. Let $\alpha<\omega_{1}$. Suppose $\mathcal{V}$ is a nonprincipal ultrafilter (without loss of generality on $\mathbb{N}$ ) such that $\mathcal{V} \leq_{T} \mathcal{U}_{\alpha}$. Then there is a front $\mathcal{F}$ on $\mathcal{C}_{\alpha}$ and a function $f: \mathcal{F} \rightarrow \mathbb{N}$ such that $\mathcal{V}=f\left(\mathcal{U}_{\alpha} \uparrow \mathcal{F}\right)$.

We now introduce notation which aids in making clear the classification of ultrafilters which are Rudin-Keisler or Tukey below $\mathcal{U}_{\alpha}$.

Notation. Let $\alpha<\omega_{1}$.

(1) For each $n<\omega$, define $\mathcal{U}_{\alpha} \uparrow \mathcal{R}_{\alpha}(n)$ to be the filter on the base $\mathcal{R}_{\alpha}(n)$ generated by the sets $\mathcal{R}_{\alpha}(n) \mid X, X \in \mathcal{C}_{\alpha}$.

(2) For each $n<\omega$ and each $S \in \mathfrak{S}_{\alpha}(n)$, define $\mathcal{Y}_{S}^{\alpha}$ to be the filter on the base set $B_{S}:=\left\{\pi_{S}(u): u \in \mathcal{R}_{\alpha}(n)\right\}$ generated by the collection of sets $\pi_{S}\left(\mathcal{R}_{\alpha}(n) \mid X\right):=\left\{\pi_{S}(u): u \in \mathcal{R}_{\alpha}(n) \mid X\right\}, X \in \mathcal{C}_{\alpha}$.

(3) For $\beta \leq \alpha$, let $\mathcal{Y}_{\beta}^{\alpha}$ denote $\mathcal{Y}_{S_{\beta}}^{\alpha}$. Recall that $S_{\beta}\left(=S_{\beta}^{\alpha}\right)$ is the downward closed subset of $\mathbb{S}_{\alpha}(0)$ of order type $[\beta, \alpha+1]$; that is, $S_{\beta}=\left\{s \in \mathbb{S}_{\alpha}(0)\right.$ : $\exists \gamma \in[\beta, \alpha](\operatorname{dom}(s)=[\gamma, \alpha])\} \cup\{\emptyset\}$.

In the next proposition, theorem and corollary, we highlight the relationships between the various projection ultrafilters of the form $\mathcal{Y}_{S}^{\alpha}$, and the ultrafilters of the form $\mathcal{U}_{\alpha} \mid \mathcal{R}_{\alpha}(n)$.

Proposition 5.6. Let $\alpha<\omega_{1}$.

(1) $\mathcal{U}_{\alpha}$ is a rapid p-point.

(2) $\mathcal{U}_{\alpha} \cong \mathcal{U}_{\alpha}\left\lceil\mathcal{R}_{\alpha}(0)=\mathcal{Y}_{0}^{\alpha}\right.$.

(3) $\mathcal{Y}_{\alpha}^{\alpha}$ is a Ramsey ultrafilter.

(4) For each $n<\omega$ and $S \in \mathfrak{S}_{\alpha}(n), \mathcal{Y}_{S}^{\alpha}$ is an ultrafilter, and moreover is a rapid p-point.

(5) Suppose $m \leq n, S \in \mathfrak{S}_{\alpha}(m), T \in \mathfrak{S}_{\alpha}(n)$, and $S \cong T$. Then $\mathcal{Y}_{S}^{\alpha} \cong \mathcal{Y}_{T}^{\alpha}$.

(6) If $S \cong \mathbb{S}_{\alpha}(k)$, then $\mathcal{Y}_{S}^{\alpha} \cong \mathcal{Y}_{\mathbb{S}_{\alpha}(k)}^{\alpha}=\mathcal{U}_{\alpha} \uparrow \mathcal{R}_{\alpha}(k)$.

Proof. (1) follows from Theorem 1.1. To see (2), recall that the map $g:\left[\mathbb{T}_{\alpha}\right] \rightarrow$ $\mathcal{R}_{\alpha}(0)$, given by $g(t)=\left\{s \in \mathbb{T}_{\alpha}: s \sqsubseteq t\right\}$ for each $t \in\left[\mathbb{T}_{\alpha}\right]$, yields an isomorphism from $\mathcal{U}_{\alpha}$ to $\mathcal{U}_{\alpha}\left\lceil\mathcal{R}_{\alpha}(0)\right.$. The equality follows from the fact that $\pi_{\mathbb{S}_{\alpha}(0)}$ is the identity map on $\mathcal{R}_{\alpha}(0)$.

(3) follows from the fact that the projection $\pi_{S_{\alpha}}$ on $\mathcal{R}_{\alpha}(0)$ yields an isomorphic copy of the Ellentuck space. Hence, $\mathcal{Y}_{\alpha}^{\alpha}$ is Ramsey for the Ellentuck space, which yields that $\mathcal{Y}_{\alpha}^{\alpha}$ is a Ramsey ultrafilter.

(4) Let $S \in \mathfrak{S}_{\alpha}(n)$. Let $V$ be any subset of $B_{S}$, and let $\mathcal{H}=\left\{a \in \mathcal{A R}_{n+1}\right.$ : $\left.\pi_{S}(a(n)) \in V\right\}$. Since $\mathcal{U}_{\alpha}$ is Ramsey for $\mathcal{R}_{\alpha}$, there is an $X \in \mathcal{C}_{\alpha}$ such that either $\mathcal{A R}_{n+1}^{\alpha} \mid X \subseteq \mathcal{H}$ or else $\mathcal{A R}_{n+1}^{\alpha} \mid X \cap \mathcal{H}=\emptyset$. In the first case, $V \in \mathcal{Y}_{S}^{\alpha}$, and in the second case, $B_{S} \backslash V \in \mathcal{Y}_{S}^{\alpha}$. Thus, $\mathcal{Y}_{S}^{\alpha}$ is an ultrafilter.

Suppose $U_{0} \supseteq U_{1} \supseteq \ldots$ is a decreasing sequence of elements of $\mathcal{Y}_{S}^{\alpha}$. For each $k<\omega$, there is some $X_{k} \in \mathcal{C}_{\alpha}$ for which $\pi_{S}\left(\mathcal{R}_{\alpha}(n) \mid X_{k}\right) \subseteq U_{k}$. We may take $\left(X_{k}\right)_{k<\omega}$ to be a $\leq_{\alpha}$-decreasing sequence. Since $\mathcal{U}_{\alpha}$ is selective for $\mathcal{R}_{\alpha}$, there is an $X \in \mathcal{C}_{\alpha}$ such that $X / r_{k}^{\alpha}(X) \leq_{\alpha} X_{k}$, for each $k<\omega$. Then $\pi_{S}\left(\mathcal{R}_{\alpha}(n) \mid X\right) \subseteq^{*} \pi_{S}\left(\mathcal{R}_{\alpha}(n) \mid X_{k}\right)$, for each $k<\omega$. Thus, $\mathcal{Y}_{S}^{\alpha}$ is a p-point.

That $\mathcal{Y}_{S}^{\alpha}$ is rapid follows from the fact that $\mathcal{U}_{\alpha} \uparrow \mathcal{R}_{\alpha}(n)$ is rapid. To see this, let $h: \omega \rightarrow \omega$ be a strictly increasing function. Linearly order $\mathcal{R}_{\alpha}(n)$ so that all 
members of $\mathcal{R}_{\alpha}(n) \mid \mathbb{T}_{\alpha}(k)$ appear before all members of $\mathcal{R}_{\alpha}(n) \mid \mathbb{T}_{\alpha}(k+1)$ for all $k \geq n$. For any tree $u \subseteq \mathbb{T}_{\alpha}$, let $m(u)$ denote the least $l$ such that $\langle l\rangle \in u$. For each $X \in \mathcal{C}_{\alpha}$, there is a $Y \leq_{\alpha} X$ such that $m(Y(n))>h(1), m(Y(n+1))>$ $h\left(1+\left|\mathcal{R}_{\alpha}(n)\right| \mathbb{T}_{\alpha}(n+1) \mid\right)$, and in general, for $k \geq n$,

$$
m(Y(k))>h\left(\Sigma_{n \leq i \leq k}\left|\mathcal{R}_{\alpha}(n)\right| \mathbb{T}_{\alpha}(i) \mid\right) .
$$

Since $\mathcal{U}_{\alpha}$ is selective for $\mathcal{R}_{\alpha}$, there is a $Y \in \mathcal{C}_{\alpha}$ with this property, which yields that $\mathcal{U}_{\alpha} \uparrow \mathcal{R}_{\alpha}(n)$ is rapid for the function $h$. Since for each $u \in \mathcal{R}_{\alpha}(n),\left|\pi_{S}(u)\right| \leq|u|$, it follows that $\pi_{S}\left(\mathcal{R}_{\alpha}(n) \mid Y\right)$ witnesses that $\mathcal{Y}_{S}^{\alpha}$ is rapid for the function $h$. Since $h$ was arbitrary, (4) holds.

(5) Suppose that $m \leq n, S \in \mathfrak{S}_{\alpha}(m), T \in \mathfrak{S}_{\alpha}(n)$, and $S \cong T$. Then $B_{T} \subseteq B_{S}$. Moreover, there is an $X \in \mathcal{C}_{\alpha}$ such that $B_{S} \mid X \subseteq B_{T}$. Thus, modulo negligible subsets of the bases, $\mathcal{Y}_{S}^{\alpha}$ is actually equal to $\mathcal{Y}_{T}^{\alpha}$. The identity map on $B_{T}$ witnesses that $\mathcal{Y}_{S}^{\alpha} \leq{ }_{R K} \mathcal{Y}_{T}^{\alpha}$. Given $X \in \mathcal{C}_{\alpha}$ such that $B_{S} \mid X \subseteq B_{T}$ and $\mathbb{T}_{\alpha} \backslash X$ is infinite, the identity map on $B_{S} \mid X$ witnesses that $\mathcal{Y}_{T}^{\alpha} \leq_{R K} \mathcal{Y}_{S}^{\alpha}$. (6) follows from (5).

For $S$ and $T$ downward closed subsets of $\mathbb{S}_{\alpha}$, we say that $S$ embeds into $T$, or $S$ is isomorphic to a subset of $T$, if there is an injection $\iota: S \rightarrow T$ which preserves lexicographic ordering (recall Definition 2.5) and such that the image $\iota(S)$ is downward closed in $T$.

Theorem 5.7. Let $m, n<\omega$, and let $S \in \mathfrak{S}_{\alpha}(m)$ and $T \in \mathfrak{S}_{\alpha}(n)$.

(1) If $S$ embeds into $T$, then $\mathcal{Y}_{S}^{\alpha} \leq_{R K} \mathcal{Y}_{T}^{\alpha}$.

(2) If $\mathcal{V} \leq_{R K} \mathcal{Y}_{T}^{\alpha}$, then $\mathcal{V} \cong \mathcal{Y}_{T^{\prime}}^{\alpha}$ for some $T^{\prime} \subseteq T$ such that $T^{\prime} \in \mathfrak{S}_{\alpha}(n)$.

(3) If $\mathcal{Y}_{S}^{\alpha} \leq_{R K} \mathcal{Y}_{T}^{\alpha}$, then $S$ embeds into $T$.

(4) $\mathcal{Y}_{S}^{\alpha} \cong \mathcal{Y}_{T}^{\alpha}$ iff $S \cong T$

Proof. (1) Suppose that $S$ is isomorphic to a subset $T^{\prime} \subseteq T$. By Proposition 5.6 (5), $\mathcal{Y}_{S}^{\alpha} \cong \mathcal{Y}_{T^{\prime}}^{\alpha}$. The projection map $\pi_{T^{\prime}}$ from $B_{T}$ to $B_{T^{\prime}}$ witnesses that $\mathcal{Y}_{T^{\prime}}^{\alpha} \leq_{R K} \mathcal{Y}_{T}^{\alpha}$.

(2) Suppose $\mathcal{V} \leq_{R K} \mathcal{Y}_{T}^{\alpha}$, and without loss of generality, assume that $\omega$ is the base set for $\mathcal{V}$. Let $\theta: B_{T} \rightarrow \omega$ witness $\mathcal{V} \leq_{R K} \mathcal{Y}_{T}^{\alpha} ; \operatorname{so} \theta\left(\mathcal{Y}_{T}^{\alpha}\right)=\mathcal{V}$. Let $f=\theta \circ \pi_{T}$ so that $f: \mathcal{R}_{\alpha}(n) \rightarrow \omega$. By the Canonization Theorem 4.22 for $\mathcal{R}_{\alpha}(n)$ and the definition of $\mathcal{U}_{\alpha}$, there is a $C \in \mathcal{C}_{\alpha}$ and a $T^{\prime} \in \mathfrak{S}_{\alpha}(n)$ such that for all $u, v \in \mathcal{R}_{\alpha}(n) \mid C$, $f(u)=f(v)$ iff $\pi_{T^{\prime}}(u)=\pi_{T^{\prime}}(v)$. Thus, there is a bijection between $f^{\prime \prime} \mathcal{R}_{\alpha}(n) \mid C$ and $\pi_{T^{\prime}}^{\prime \prime} \mathcal{R}_{\alpha}(n) \mid C$.

Suppose that $T^{\prime} \backslash T \neq \emptyset$. Then there are $u, v \in \mathcal{R}_{\alpha}(n) \mid C$ such that $\pi_{T}(u)=\pi_{T}(v)$ but $\pi_{T^{\prime}}(u) \neq \pi_{T^{\prime}}(v) . \quad \pi_{T}(u)=\pi_{T}(v)$ implies that $\theta\left(\pi_{T}(u)\right)=\theta\left(\pi_{T}(v)\right)$, which implies that $f(u)=f(v)$. However, $\pi_{T^{\prime}}(u) \neq \pi_{T^{\prime}}(v)$ implies that $f(u) \neq f(v)$, a contradiction. Thus $T^{\prime} \subseteq T$. Hence, $\mathcal{V}=\theta\left(\mathcal{Y}_{T}^{\alpha}\right)=\theta\left(\pi_{T}\left(\mathcal{U}_{\alpha} \uparrow \mathcal{R}_{\alpha}(n)\right)\right)=$ $f\left(\mathcal{U}_{\alpha}\left\lceil\mathcal{R}_{\alpha}(n)\right) \cong \pi_{S}\left(\mathcal{U}_{\alpha}\left\lceil\mathcal{R}_{\alpha}(n)\right)=\mathcal{Y}_{S}^{\alpha}\right.\right.$.

(3) Suppose that $\theta: B_{T} \rightarrow B_{S}$ witnesses that $\mathcal{Y}_{S}^{\alpha} \leq_{R K} \mathcal{Y}_{T}^{\alpha}$. By (2), there is a $T^{\prime} \subseteq T$ such that $\mathcal{Y}_{S}^{\alpha} \cong \mathcal{Y}_{T^{\prime}}^{\alpha}$. Let $\theta: B_{T^{\prime}} \rightarrow B_{S}$ be an isomorphism witnessing this. Let $f: \mathcal{R}_{\alpha}(n) \rightarrow B_{S}$ by letting $f=\theta \circ \pi_{T^{\prime}}$. By Theorem 4.3 and the definition of $\mathcal{U}_{\alpha}$, there is some $T^{\prime \prime} \in \mathfrak{S}_{\alpha}(n)$ and $U \in \mathcal{C}_{\alpha}$ such that for all $u, v \in \mathcal{R}_{\alpha}(n) \mid U$, $f(u)=f(v)$ iff $\pi_{T^{\prime \prime}}(u)=\pi_{T^{\prime \prime}}(v)$. We claim that $T^{\prime}=T^{\prime \prime} \cong S . T^{\prime}$ must equal $T^{\prime \prime}$, since $f$ is injective. Moreover, $\pi_{T^{\prime \prime}}(u)=\pi_{T^{\prime \prime}}(v)$ iff $\pi_{S}(u)=\pi_{S}(v)$. Hence, $T^{\prime \prime} \cong S$.

(4) If $S \cong T$, then $\mathcal{Y}_{S}^{\alpha} \cong \mathcal{Y}_{T}^{\alpha}$ by Proposition [5.6 (5). If $\mathcal{Y}_{S}^{\alpha} \cong \mathcal{Y}_{T}^{\alpha}$, then by applying (3) twice, we find that $S$ and $T$ are isomorphic to subsets of each other. Hence, $S \cong T$. 
The next corollary follows immediately from Proposition 5.6] and Theorem 5.7. thus, recovering Laflamme's Theorem 1.1 (3).

Corollary 5.8. $\left\langle\mathcal{Y}_{\beta}^{\alpha}: \beta \leq \alpha\right\rangle$ forms a strictly decreasing chain of nonprincipal rapid p-points in the Rudin-Keisler ordering, with $\mathcal{Y}_{0}^{\alpha}$ Rudin-Keisler maximal and $\mathcal{Y}_{\alpha}^{\alpha}$ Rudin-Keisler minimal in the chain. Moreover, this chain is maximal within the ordering of nonprincipal ultrafilters Rudin-Keisler reducible to $\mathcal{U}_{\alpha}$.

We will extend the previous corollary to the setting of Tukey reducibility in Theorem 5.13 .

Theorem 5.9. Let $n<\omega$ and $S \in \mathfrak{S}_{\alpha}(n)$. Let $\beta \leq \alpha$ be minimal such that $S_{\beta}$ embeds into $S$. Then $\mathcal{Y}_{S}^{\alpha} \equiv_{T} \mathcal{Y}_{\beta}^{\alpha}$.

Proof. Let $n<\omega$ and $S \in \mathfrak{S}_{\alpha}(n)$. Let $\beta \leq \alpha$ be minimal such that $S$ contains an isomorphic copy of $S_{\beta}$, call it $S^{\prime}$. Thus, $S^{\prime}$ is a downward closed chain in $S$ with largest order type among all chains in $S$, namely o.t. $([\beta, \alpha])$. The projection map $\pi_{S^{\prime}}: B_{S} \rightarrow B_{S^{\prime}}$ witnesses that $\mathcal{Y}_{S^{\prime}}^{\alpha} \leq_{R K} \mathcal{Y}_{S}^{\alpha}$. Since $\mathcal{Y}_{S^{\prime}}^{\alpha}$ is isomorphic to $\mathcal{Y}_{\beta}^{\alpha}$, we have that $\mathcal{Y}_{\beta}^{\alpha} \leq_{R K} \mathcal{Y}_{S}^{\alpha}$. Hence, $\mathcal{Y}_{\beta}^{\alpha} \leq_{T} \mathcal{Y}_{S}^{\alpha}$.

For the reverse inequality, first note that for each $X \in \mathcal{C}_{\alpha}$, from $\pi_{S_{\beta}}\left(\mathcal{R}_{\alpha}(0) \mid X\right)$ one can reconstruct $\pi_{S}\left(\mathcal{R}_{\alpha}(n) \mid X\right)$, since $\beta$ is minimal such that there is a member $s \in S$ with $\operatorname{dom}(s)=[\beta, \alpha]$. Thus, for each $X \in \mathcal{C}_{\alpha}$, define $g\left(\pi_{S_{\beta}}\left(\mathcal{R}_{\alpha}(0) \mid X\right)\right)=$ $\pi_{S}\left(\mathcal{R}_{\alpha}(n) \mid X\right)$. Then $g$ maps a cofinal subset of $\mathcal{Y}_{\beta}^{\alpha}$ cofinally and monotonically into $\mathcal{Y}_{S}^{\alpha}$. Therefore, $\mathcal{Y}_{S}^{\alpha} \leq_{T} \mathcal{Y}_{\beta}^{\alpha}$.

Now we are ready to prove the main theorem of this section, the classification of all Rudin-Keisler types of ultrafilters Tukey reducible to $\mathcal{U}_{\alpha}$. The following notion of an ultrafilter of a $\overrightarrow{\mathcal{W}}$-tree encompasses the notion of iterated Fubini products of ultrafilters.

Definition 5.10. Let $\hat{\mathcal{T}}$ be a well-founded tree, let $\mathcal{T}$ denote the set of maximal nodes in $\hat{\mathcal{T}}$, and suppose that each $t \in \hat{\mathcal{T}} \backslash \mathcal{T}$ has infinitely many immediate successors in $\hat{\mathcal{T}}$. For each $t \in \hat{\mathcal{T}} \backslash \mathcal{T}$, let $\mathcal{W}_{t}$ be an ultrafilter on the base set consisting of all immediate successors of $t$ in $\hat{\mathcal{T}}$. Let $\overrightarrow{\mathcal{W}}$ denote $\left(\mathcal{W}_{t}: t \in \hat{\mathcal{T}} \backslash \mathcal{T}\right)$. Then a $\overrightarrow{\mathcal{W}}$-tree is a tree $T \subseteq \hat{\mathcal{T}}$ such that for each $t \in T \cap(\hat{\mathcal{T}} \backslash \mathcal{T})$, the collection of immediate successors of $t$ in $T$ is a member of the ultrafilter $\mathcal{W}_{t}$.

Theorem 5.11. Suppose $\mathcal{V}$ is a nonprincipal ultrafilter and $\mathcal{V} \leq_{T} \mathcal{U}_{\alpha}$. Then $\mathcal{V}$ is isomorphic to an ultrafilter of $\overrightarrow{\mathcal{W}}$-trees, where $\hat{\mathcal{T}} \backslash \mathcal{T}$ is a well-founded tree, $\overrightarrow{\mathcal{W}}=$ $\left(\mathcal{W}_{t}: t \in \hat{\mathcal{T}} \backslash \mathcal{T}\right)$, and each $\mathcal{W}_{t}$ is exactly $\mathcal{Y}_{S}^{\alpha}$ for some $n<\omega$ and $S \in \mathfrak{S}_{\alpha}(n)$.

Proof. The proof is so similar to the proof of Theorem 5.10 in [5] that we only give a sketch of the proof, providing the few changes here. By Proposition 5.5, there is a front $\mathcal{F}$ on $\mathcal{C}_{\alpha}$ and a function $f: \mathcal{F} \rightarrow \mathbb{N}$ such that $\mathcal{V}=f\left(\mathcal{U}_{\alpha} \mid \mathcal{F}\right)$. By Theorem 4.12 and the fact that $\mathcal{U}_{\alpha}$ is canonical for fronts, there is a $C \in \mathcal{C}_{\alpha}$ such that the equivalence relation induced by $f$ on $\mathcal{F} \mid C$ is canonical. Let $\varphi$ denote the function from Theorem 4.12 which canonizes $f$. If $\mathcal{F}=\{\emptyset\}$, then $\mathcal{V}$ is a principal ultrafilter, so we may assume that $\mathcal{F} \neq\{\emptyset\}$.

Let $\mathcal{T}=\{\varphi(a): a \in \mathcal{F} \mid C\}$. Define $\mathcal{W}$ to be the filter on base set $\mathcal{T}$ generated by the sets $\{\varphi(a): a \in \mathcal{F} \mid X\}, X \in \mathcal{C}_{\alpha} \mid C$. For $X \in \mathcal{C}_{\alpha} \mid C$, let $\mathcal{T} \mid X$ denote $\{\varphi(a)$ : $a \in \mathcal{F} \mid X\}$. By arguments in [5], $\mathcal{W}$ is an ultrafilter which is isomorphic to $\mathcal{V}$. Let $\hat{\mathcal{T}}$ denote the collection of all initial segments of elements of $\mathcal{T}$. Precisely, let $\hat{\mathcal{T}}$ be 
the collection of all $\varphi(a) \cap r_{i}^{\alpha}(a)$ such that $a \in \mathcal{F}|C, i \leq| a \mid$, and if $0<i<|a|$, then $S_{r_{i}^{\alpha}(a)} \neq\{\emptyset\} . \hat{\mathcal{T}}$ forms a tree under the end-extension ordering. Recall from the proof of Theorem 4.12 that for $t \in \hat{\mathcal{T}} \backslash \mathcal{T}$, for all $a, b \in \mathcal{F}$, if $j<|a|$ is maximal such that $\varphi\left(r_{j}^{\alpha}(a)\right)=t$ and $k$ is maximal such that $\varphi\left(r_{k}^{\alpha}(b)\right)=t$, then $S_{r_{j}^{\alpha}(a)}$ is isomorphic to $S_{r_{k}^{\alpha}(b)}$, and these are both not $\{\emptyset\}$.

For $t \in \hat{\mathcal{T}} \backslash \mathcal{T}$, define $\mathcal{W}_{t}$ to be the filter generated by the sets $\left\{\varphi_{r_{j}^{\alpha}(a)}(u): u \in\right.$ $\left.\mathcal{R}_{\alpha}(j) \mid X / a\right\}$, for all $a \in \mathcal{F} \mid C$ such that $t \sqsubseteq \varphi(a)$ and $j<|a|$ maximal such that $\varphi\left(r_{j}^{\alpha}(a)\right)=t$, and all $X \in \mathcal{C}_{\alpha} \mid C$. The base set for $\mathcal{W}_{t}$ is $\left\{\pi_{S_{r_{j}^{\alpha}(a)}}(u): u \in \mathcal{R}_{\alpha}(j) \mid C\right\}$. By arguments in [5, it follows that for each $t \in \hat{\mathcal{T}} \backslash \mathcal{T}, \mathcal{W}_{t}$ is an ultrafilter; moreover, for any $a \in \mathcal{F}$ and $j<|a|$ maximal such that $\varphi\left(r_{j}^{\alpha}(a)\right)=t, \mathcal{W}_{t}$ is generated by the collection of $\left\{\varphi_{r_{j}(a)}(u): u \in \mathcal{R}_{\alpha}(j) \mid X\right\}, X \in \mathcal{C}_{\alpha} \mid C$. This follows from the fact that $\mathcal{U}_{\alpha}$ is Ramsey for $\mathcal{R}_{\alpha}$.

Claim 5.12. Let $t \in \hat{\mathcal{T}} \backslash \mathcal{T}$. Then $\mathcal{W}_{t}$ equals $\mathcal{Y}_{S}^{\alpha}$ for some $n<\omega$ and $S \in \mathfrak{S}_{\alpha}(n)$.

Proof. Fix $a \in \mathcal{F} \mid C$ and $j<|a|$ with $j$ maximal such that $\varphi\left(r_{j}^{\alpha}(a)\right)=t$. Let $S$ denote $S_{r_{j}^{\alpha}(a)}$. For each $X \in \mathcal{C}_{\alpha} \mid C,\left\{\varphi_{r_{j}^{\alpha}(a)}(u): u \in \mathcal{R}_{\alpha}(j) \mid X\right\}=\pi_{S}\left(\mathcal{R}_{\alpha}(j) \mid X\right) \in$ $\mathcal{Y}_{S}^{\alpha}$. Since $\mathcal{W}_{t}$ is a nonprincipal ultrafilter, $\mathcal{W}_{t}$ must equal $\mathcal{Y}_{S}^{\alpha}$, by Fact 5.4.

Thus, $\mathcal{W}$ is the ultrafilter of $\overrightarrow{\mathcal{W}}$-trees, where $\overrightarrow{\mathcal{W}}=\left(\mathcal{W}_{t}: t \in \hat{\mathcal{T}} \backslash \mathcal{T}\right)$. This follows from the fact that for each $\overrightarrow{\mathcal{W}}$-tree $\hat{T} \subseteq \hat{\mathcal{T}},[\hat{T}]$ is a member of $\mathcal{W}$. Thus, $\mathcal{V}$ is isomorphic to the ultrafilter $\mathcal{W}$ on base set $\mathcal{T}$ generated by the $\overrightarrow{\mathcal{W}}$-trees.

Corollary 5.8 and Theorems 5.9 and 5.11 yield the analogue of Laflamme's result for the Rudin-Keisler ordering (Theorem 1.1(3)) now in the context of Tukey types.

Theorem 5.13. Let $\alpha<\omega_{1}$ and suppose $\mathcal{V}$ is a nonprincipal ultrafilter such that $\mathcal{V} \leq_{T} \mathcal{U}_{\alpha}$. Then there is a $\beta \leq \alpha$ such that $\mathcal{V} \equiv_{T} \mathcal{Y}_{\beta}^{\alpha}$. Thus, the collection of the Tukey types of all nonprincipal ultrafilters Tukey reducible to $\mathcal{U}_{\alpha}$ forms a decreasing chain of rapid p-points of order type $(\alpha+1)^{*}$.

Proof. Let $\mathcal{V}$ be a nonprincipal ultrafilter such that $\mathcal{V} \leq_{T} \mathcal{U}_{\alpha}$. Theorem 5.11 implies that $\mathcal{V}$ is isomorphic, and hence Tukey equivalent, to the ultrafilter on $\mathcal{T}$ generated by the $\overrightarrow{\mathcal{W}}$-trees, where for each $t \in \hat{\mathcal{T}} \backslash \mathcal{T}$, the ultrafilter $\mathcal{W}_{t}$ is $\mathcal{Y}_{S_{t}}^{\alpha}$ for some $n<\omega$ and some $S_{t} \in \mathfrak{S}_{\alpha}(n)$. By Theorem [5.9] for each $t$, there is a $\beta_{t} \leq \alpha$ such that $\mathcal{Y}_{S_{t}}^{\alpha} \equiv_{T} \mathcal{Y}_{\beta_{t}}^{\alpha}$. It follows that $\mathcal{V}$ is Tukey equivalent to the ultrafilter of $\left\langle\mathcal{Y}_{\beta_{t}}^{\alpha}: t \in \hat{\mathcal{T}} \backslash \mathcal{T}\right\rangle$-trees. By induction on the lexicographical rank of $\mathcal{T}$, one concludes that the ultrafilter of $\left\langle\mathcal{Y}_{\beta_{t}}^{\alpha}: t \in \hat{\mathcal{T}} \backslash \mathcal{T}\right\rangle$-trees is Tukey equivalent to $\mathcal{Y}_{\beta}^{\alpha}$, where $\beta=\min \left\{\beta_{t}: t \in \hat{\mathcal{T}} \backslash \mathcal{T}\right\}$.

Now suppose that $\gamma<\beta \leq \alpha$ and suppose toward a contradiction that $\mathcal{Y}_{\beta}^{\alpha} \leq_{T}$ $\mathcal{Y}_{\gamma}^{\alpha}$. Then there is a continuous monotone cofinal map $h: \mathcal{Y}_{\gamma}^{\alpha} \rightarrow \mathcal{Y}_{\beta}^{\alpha}$, since $\mathcal{Y}_{\gamma}^{\alpha}$ and $\mathcal{Y}_{\beta}^{\alpha}$ are p-points. Since $\mathcal{Y}_{\gamma}^{\alpha} \leq_{R K} \mathcal{U}_{\alpha}$, let $g: \mathbb{T}_{\alpha} \rightarrow B_{S_{\gamma}}$ be such that $g\left(\mathcal{U}_{\alpha}\right)=\mathcal{Y}_{\gamma}^{\alpha}$. Then $h \circ g: \mathcal{U}_{\alpha} \rightarrow \mathcal{Y}_{\beta}^{\alpha}$ is a continuous monotone cofinal map. By Proposition [5.5, there is a front $\mathcal{F}$ and a function $f: \mathcal{F} \rightarrow B_{S_{\beta}}$ such that $\mathcal{Y}_{\beta}^{\alpha}=f\left(\left\langle\mathcal{U}_{\alpha}\lceil\mathcal{F}\rangle\right)\right.$. By Theorem 4.12, there is a $C \in \mathcal{C}_{\alpha}$ such that $f \uparrow \mathcal{F} \mid C$ is canonical, witnessed by the inner function $\varphi$. Noting that for each $X \in \mathcal{C}_{\alpha} \mid C, f(\mathcal{F} \mid X) \subseteq h \circ g(X)$ and $g(X) \subseteq B_{\gamma}$, we see that $\varphi$ cannot distinguish between members $a, b \in \mathcal{F}$ for which $\pi_{S_{\gamma}}(a)=\pi_{S_{\gamma}}(b)$, a contradiction to $f\left(\left\langle\mathcal{U}_{\alpha}\lceil\mathcal{F}\rangle\right)=\mathcal{Y}_{\beta}^{\alpha}\right.$. 
By Corollary [5.8, the $\mathcal{Y}_{\beta}^{\alpha}, \beta \leq \alpha$, form a maximal chain in the Tukey ordering of ultrafilters Tukey reducible to $\mathcal{U}_{\alpha}$.

The second half follows from Theorems 5.9 and 5.11 .

Remark 5.14. It follows from Theorem 5.13 that the Tukey equivalence class of $\mathcal{Y}_{\beta}^{\alpha}$ consists exactly of those ultrafilters which are isomorphic to some ultrafilter of $\overrightarrow{\mathcal{W}}$-trees, where for each $t \in \hat{\mathcal{T}} \backslash \mathcal{T}, \mathcal{W}_{t} \cong \mathcal{Y}_{S_{t}}^{\alpha}$ for some $S_{t}$ satisfying the following: for each $t \in \hat{\mathcal{T}} \backslash \mathcal{T}$, if $S_{\gamma}$ embeds into $S_{t}$, then $\gamma \geq \beta$; and for at least one $t \in \hat{\mathcal{T}} \backslash \mathcal{T}$, $S_{\beta}$ embeds into $S_{t}$.

The theorems in this section make clear exactly what the Rudin-Keisler structures are within the Tukey types of the ultrafilters $\mathcal{Y}_{\beta}^{\alpha}$, and more generally, what the Rudin-Keisler structures are among all ultrafilters Tukey reducible to $\mathcal{Y}_{\beta}^{\alpha}$. We point some of these out here.

The Rudin-Keisler structure in $\left[\mathcal{U}_{\alpha}\right]_{T}$. The Tukey type of $\mathcal{U}_{\alpha}$ consists exactly of all ultrafilters isomorphic to a tree ultrafilter of the sort in Theorem 5.11 in which for every $X \in \mathcal{C}_{\alpha}$, there is at least one $a \in \mathcal{F} \mid X$ and an $i<|a|$ such that $S_{0}^{\alpha}\left(=\mathbb{S}_{\alpha}(0)\right)$ embeds into $S_{r_{i}^{\alpha}(a)}$. Let $\mathfrak{S}_{0}^{\alpha}$ denote the collection of all $S \in \bigcup_{n<\omega} \mathfrak{S}_{\alpha}(n)$ for which $S_{0}^{\alpha}$ embeds into $S$.

The isomorphism types of the p-points in $\left[\mathcal{U}_{\alpha}\right]_{T}$ are simply classified as follows: The p-points Tukey equivalent to $\mathcal{U}_{\alpha}$ are, up to isomorphism, exactly the $\mathcal{Y}_{S}^{\alpha}$, where $S \in \mathfrak{S}_{0}^{\alpha}$. Thus, the structures $S \in \bigcup_{m<\omega} \mathfrak{S}_{\alpha}(m)$ which have at least one node with domain $[0, \alpha]$ completely determine the strucure of the isomorphism classes of ppoints in $\left[\mathcal{U}_{\alpha}\right]_{T}$. In particular, there are exactly $\aleph_{0}$ Rudin-Keisler types of p-points inside $\left[\mathcal{U}_{\alpha}\right]_{T}$, for each $1 \leq \alpha<\omega_{1}$. Recalling that all $\mathcal{Y}_{S}^{\alpha}$ are also rapid, we see that every p-point Tukey equivalent to $\mathcal{U}_{\alpha}$ is rapid.

Let $S, S^{\prime}, T, T^{\prime} \in \mathfrak{S}_{0}^{\alpha}$. If $S$ embeds into $T$, then $\mathcal{Y}_{S}^{\alpha} \leq_{R K} \mathcal{Y}_{T}^{\alpha}$. If neither $S$ nor $T$ embeds into the other, then $\mathcal{Y}_{S}^{\alpha}$ and $\mathcal{Y}_{T}^{\alpha}$ are Rudin-Keisler incomparable. If $S^{\prime}$ embeds into both $S$ and $T$, then $\mathcal{Y}_{S^{\prime}}^{\alpha} \leq_{R K} \mathcal{Y}_{S}^{\alpha}, \mathcal{Y}_{T}^{\alpha}$. If $S$ and $T$ both embed into $T^{\prime}$, then $\mathcal{Y}_{S}^{\alpha}, \mathcal{Y}_{T}^{\alpha} \leq_{R K} \mathcal{Y}_{T^{\prime}}^{\alpha}$. We note that for all pairs $S, T \in \mathfrak{S}_{0}^{\alpha}$, there are $S^{\prime}, T^{\prime} \in \mathfrak{S}_{0}^{\alpha}$ such that $S^{\prime}$ embeds into both $S$ and $T$, and both $S$ and $T$ embed into $T^{\prime}$. Hence, the isomorphism classes of p-points in $\left[\mathcal{U}_{\alpha}\right]_{T}$ form a sort of lattice-like structure in the sense that upper bounds and lower bounds for any finite collection of $\mathcal{Y}_{S}^{\alpha}$ exist.

For $\alpha=1$, the structure of the Rudin-Keisler types of p-points in $\left[\mathcal{U}_{1}\right]_{T}$ is simply the following increasing $\omega$-chain: $\mathcal{Y}_{\mathbb{S}_{1}(0)}^{1}<_{R K} \mathcal{Y}_{\mathbb{S}_{1}(1)}^{1}<_{R K} \mathcal{Y}_{\mathbb{S}_{1}(2)}^{1}<_{R K} \ldots$ For $\alpha>1$, the structure is more complex and is not truly a lattice, as least upper bounds and greatest lower bounds of $S$ and $T$ in $\mathfrak{S}_{0}^{\alpha}$ need not exist. For example, let $S, T \in \mathfrak{S}_{0}^{2}$ be the following: Let $S$ and $T$ be the downward-closed substructures of $\mathbb{S}_{2}(4)$ determined by their terminal nodes $\{(0,28),(0,29),(1,8),(0,45)\}$ and $\{(1,7),(0,36),(0,37),(0,45),(0,46),(1,10)\}$, respectively. Then there is no least upper bound and no greatest lower bound of $S$ and $T$ in $\mathfrak{S}_{0}^{2}$. However, they do have upper and lower bounds, for instance, $\mathbb{S}_{2}(4)$ and $S_{0}^{2}$, respectively. Thus, among the p-points in $\left[\mathcal{U}_{2}\right]_{T}$, there are upper $\left(\mathcal{Y}_{\mathbb{S}_{2}(4)}^{2}\right)$ and lower $\left(\mathcal{Y}_{S_{0}^{2}}^{2}\right)$ bounds of $\mathcal{Y}_{S}^{2}$ and $\mathcal{Y}_{T}^{2}$. However, there is no Rudin-Keisler least upper bound of $\mathcal{Y}_{S}^{2}$ and $\mathcal{Y}_{T}^{2}$, and no Rudin-Keisler greatest lower bound of $\mathcal{Y}_{S}^{2}$ and $\mathcal{Y}_{T}^{2}$ among the p-points in $\left[\mathcal{U}_{2}\right]_{T}$.

When $\omega \leq \alpha<\omega_{1}$, there are infinite antichains in the Rudin-Keisler structure of the p-points in $\left[\mathcal{U}_{\alpha}\right]_{T}$. For example, letting $\alpha=\omega$, for each $n<\omega$, let $T_{0}=\mathbb{S}_{\omega}(1)$, let $T_{1}$ be the substructure of $\mathbb{S}_{\omega}(2)$ determined by the nodes $\{(1,2),(1,3)\}$ to be 
all nodes either below both of these, or else in the leftmost branch in $\mathbb{S}_{\omega}(2)$ above one of these. In general, let $T_{n}$ be the substructure of $\mathbb{S}_{\omega}(n+1)$ determined by the nodes $\{(n, n+1),(n, n+2)\}$ and all nodes either below them or else in the leftmost branch above one of them. Then the p-points $\mathcal{Y}_{T_{n}}^{\omega}, n<\omega$, form an antichain in $\left[\mathcal{U}_{\omega}\right]_{T}$.

We now discuss the structure of all isomorphism classes in $\left[\mathcal{U}_{\alpha}\right]_{T}$. The Tukey type of $\mathcal{U}_{\alpha}$ contains all isomorphism types of countable iterations of Fubini products of $\mathcal{U}_{\alpha}$. Hence, $\left[\mathcal{U}_{\alpha}\right]_{T}$ contains a Rudin-Keisler strictly increasing chain of order type $\omega_{1}$. It also contains a rich array of ultrafilters which are Rudin-Keisler incomparable. As mentioned above, for any $S, T \in \mathfrak{S}_{0}^{\alpha}$ such that neither $S$ nor $T$ embeds into the other, the p-points $\mathcal{Y}_{S}^{\alpha}$ and $\mathcal{Y}_{T}^{\alpha}$ are Rudin-Keisler incomparable and are Tukey equivalent to $\mathcal{U}_{\alpha}$. By arguments using the Abstract Ellentuck Theorem, one sees that, for each $n \geq 2, \mathcal{U}_{\alpha} \cdot \mathcal{U}_{\alpha}$ and $\mathcal{Y}_{\mathbb{S}_{\alpha}(n)}^{\alpha}$ are Rudin-Keisler incomparable. Furthermore, whenever $S, S^{\prime}, T, T^{\prime} \in \mathfrak{S}_{0}^{\alpha}, S$ does not embed into $S^{\prime}$, and $T^{\prime}$ does not embed into $T$, then $\mathcal{Y}_{S}^{\alpha} \cdot \mathcal{Y}_{T}^{\alpha}$ and $\mathcal{Y}_{S^{\prime}}^{\alpha} \cdot \mathcal{Y}_{T^{\prime}}^{\alpha}$ are Rudin-Keisler incomparable. In particular, whenever $k<l<m<n, \mathcal{Y}_{\mathbb{S}_{\alpha}(k)}^{\alpha} \cdot \mathcal{Y}_{\mathbb{S}_{\alpha}(n)}^{\alpha}$ and $\mathcal{Y}_{\mathbb{S}_{\alpha}(l)}^{\alpha} \cdot \mathcal{Y}_{\mathbb{S}_{\alpha}(m)}^{\alpha}$ are Tukey equivalent to $\mathcal{U}_{\alpha}$ but are Rudin-Keisler incomparable with each other. Further examples of Rudin-Keisler incomparable ultrafilters in $\left[\mathcal{U}_{\alpha}\right]_{T}$ can be made similarly, using iterated Fubini products and $\mathcal{Y}_{S}^{\alpha}, S \in \mathfrak{S}_{0}^{\alpha}$.

The Rudin-Keisler structure in $\left[\mathcal{Y}_{\beta}^{\alpha}\right]_{T}, \beta \leq \alpha$. This is quite similar to the structure of the isomorphism types in $\left[\mathcal{U}_{\alpha}\right]_{T}$. Since $\mathcal{U}_{\alpha} \cong \mathcal{Y}_{0}^{\alpha}$, the above paragraphs take care of the case when $\beta=0$. In what follows, let $\mathfrak{S}_{\beta}^{\alpha}$ denote the collection of all $S \in \bigcup_{n<\omega} \mathfrak{S}_{\alpha}(n)$ such that $S_{\beta}^{\alpha}$ embeds into $S$, but $S_{\gamma}^{\alpha}$ does not embed into $S$ for any $\gamma<\beta$.

For each $\beta \leq \alpha$, the Tukey class of $\mathcal{Y}_{\beta}^{\alpha}$ consists, up to isomorphism, of all tree ultrafilters of the sort in Theorem 5.11 in which (a) each node branches into some p-point $\mathcal{Y}_{S}^{\alpha}$ for some $S \in \mathfrak{S}_{\beta}^{\alpha}$, (b) for every $X \in \mathcal{C}_{\alpha}$, there is at least one $a \in \mathcal{F} \mid X$ and an $i<|a|$ such that $S_{\beta}^{\alpha}$ embeds into $S_{r_{i}^{\alpha}(a)}$, and (c) there is a $Y \in \mathcal{C}_{\alpha}$ such that for each $a \in \mathcal{F}|Y, i<| a \mid$, and $\gamma<\beta, S_{\gamma}^{\alpha}$ does not embed into $S_{r_{i}^{\alpha}(a)}$.

The p-points Tukey equivalent to $\mathcal{Y}_{\beta}^{\alpha}$ are, up to isomorphism, exactly the $\mathcal{Y}_{S}^{\alpha}$, where $S \in \mathfrak{S}_{\beta}^{\alpha}$. Thus, the structures in $\mathfrak{S}_{\beta}^{\alpha}$ determine the structure of the isomorphims classes of p-points which are Tukey equivalent to $\mathcal{Y}_{\beta}^{\alpha}$. There are RudinKeisler strictly decreasing chains of order type o.t. $[\beta, \alpha]$, and Rudin-Keisler strictly increasing chains of length $\omega$. Furthermore, there are many Rudin-Keisler incomparable p-points: for any $S, T \in \mathfrak{S}_{\beta}^{\alpha}$, if neither $S$ nor $T$ embeds into the other, then $\mathcal{Y}_{S}^{\alpha}$ and $\mathcal{Y}_{T}^{\alpha}$ are Rudin-Keisler incomparable. When $\alpha<\omega$, every Rudin-Keisler antichain of p-points in $\left[\mathcal{Y}_{\beta}^{\alpha}\right]_{T}$ is finite. When $\alpha \geq \omega$, there are Rudin-Keisler antichains of p-points in $\left[\mathcal{Y}_{\beta}^{\alpha}\right]_{T}$ of size $\aleph_{0}$.

More generally, there is a rich array of Rudin-Keisler incomparable ultrafilters in $\left[\mathcal{Y}_{\beta}^{\alpha}\right]_{T}$. For instance, if $S, T \in \mathfrak{S}_{\beta}^{\alpha}, S^{\prime} \in \mathfrak{S}_{\gamma}^{\alpha}$, and $T^{\prime} \in \mathfrak{S}_{\delta}^{\alpha}$, for some $\gamma, \delta \in[\beta, \alpha]$, and all of $S, S^{\prime}, T, T^{\prime}$ do not embed in the others, then $\mathcal{Y}_{S}^{\alpha} \cdot \mathcal{Y}_{S^{\prime}}^{\alpha}$ and $\mathcal{Y}_{T}^{\alpha} \cdot \mathcal{Y}_{T^{\prime}}^{\alpha}$ are Rudin-Keisler incomparable.

The Rudin-Keisler and Tukey structures in the collection of all ultrafilters Tukey reducible to $\mathcal{Y}_{\beta}^{\alpha}$, for $\beta \leq \alpha$. The collection of all p-points Tukey reducible to $\mathcal{U}_{\alpha}$ consists, up to isomorphism, exactly of all $\mathcal{Y}_{S}^{\alpha}$, where $S \in \bigcup_{n<\omega} \mathfrak{S}_{\alpha}(n)$. It includes the following Rudin-Keisler strictly decreasing chain of rapid p-points 
of order type $\alpha+1: \mathcal{Y}_{0}^{\alpha}>_{R K} \mathcal{Y}_{1}^{\alpha}>_{R K} \cdots>_{R K} \mathcal{Y}_{\alpha}^{\alpha}$. Since each of the $\mathcal{Y}_{\beta}^{\alpha}$, $\beta \leq \alpha$, is a p-point, none of the ultrafilters in this chain is a Fubini product of any other ultrafilters. Moreover, it follows from Theorem 5.11 that this chain is Rudin-Keisler-maximal within the collection of ultrafilters Tukey reducible to $\mathcal{U}_{\alpha}$. This chain is also Tukey-maximal decreasing within this collection, by Theorem 5.13. The collection of all ultrafilters Tukey reducible to $\mathcal{U}_{\alpha}$ consists exactly of all tree ultrafilters which branch into p-points from among $\mathcal{Y}_{S}^{\alpha}, S \in \bigcup_{n<\omega} \mathfrak{S}_{\alpha}(n)$.

For any $\beta \leq \alpha$, the collection of all ultrafilters Tukey reducible to $\mathcal{Y}_{\beta}^{\alpha}$ includes the Rudin-Keisler (and Tukey) strictly decreasing chain of rapid p-points $\mathcal{Y}_{\beta}^{\alpha}>_{R K}$ $\cdots>_{R K} \mathcal{Y}_{\alpha}^{\alpha}$. In addition, it contains many ultrafilters which are Rudin-Keisler incomparable. For any $\gamma<\delta<\varepsilon<\zeta \leq \beta, \mathcal{Y}_{\gamma}^{\alpha} \cdot \mathcal{Y}_{\zeta}^{\alpha}$ and $\mathcal{Y}_{\delta}^{\alpha} \cdot \mathcal{Y}_{\varepsilon}^{\alpha}$ are both Tukey reducible to $\mathcal{Y}_{\beta}^{\alpha}$, and are Rudin-Keisler incomparable with each other. More general examples may be constructed by the interested reader using iterated Fubini products of appropriate subsets of ultrafilters from among $\mathcal{Y}_{\gamma}^{\alpha}, \gamma \leq \beta$.

\section{Conclusion}

In Parts 1 and 2, we have constructed a new transfinite hierarchy of topological Ramsey spaces, $\mathcal{R}_{\alpha}, \alpha<\omega_{1}$, and proved Ramsey-classification theorems for them. We have then applied these theorems to find the structures of the Tukey types of all ultrafilters Tukey reducible to $\mathcal{U}_{\alpha}$, and even more, the isomorphism types within the Tukey types of the ultrafilters Tukey reducible to $\mathcal{U}_{\alpha}$. This work points to the following lines of questions.

Question 6.1. What other natural topological Ramsey spaces can be constructed?

For each $\alpha<\omega_{1}$, the Tukey types of all ultrafilters Tukey reducible to $\mathcal{U}_{\alpha}$ form a strictly decreasing sequence of order type $\alpha+1$.

Question 6.2. What other structures embed into the Tukey types of ultrafilters which are not at the Tukey top? In particular, what structures embed into the Tukey types of ultrafilters near the bottom of the Tukey hierarchy?

Question 6.3. To what general classes of topological Ramsey spaces can our methods be applied to prove new Ramsey-classification theorems? Does every topological Ramsey space support a Ramsey-classification theorem for equivalence relations on its barriers?

Recently, Mijares and Padilla constructed in [12] a new topological Ramsey space of infinite polyhedra, which they use to show that the group of automorphisms of the Fraïssé limit of the class of finite ordered polyhedra is extremely amenable, providing a new example of an extremely amenable group and hence, by work of Kechris, Pestov and Todorcevic, a related universal minimal flow (see [9]).

A forthcoming paper of Dobrinen, Mijares, and Trujillo provides some answers to Questions 6.1 - 6.3. In 6], for each $1 \leq n<\omega$, they construct new topological Ramsey spaces, inspired by a combination of the spaces $\mathcal{R}_{\alpha}$ and some work of Blass in [1. These spaces were built so that the structure of the Tukey types below that of the associated ultrafilter are isomorphic to a large array of countable lattices, including the finite Boolean algebras $\mathcal{P}(n), n<\omega$. Other structures of initial segments of Tukey types are the subject of current work.

In another direction answering Questions 6.2 and 6.3. Dobrinen in 3 builds new topological Ramsey spaces and classifies the structures of the Rudin-Keisler and 
Tukey types of p-points which are not weakly Ramsey, yet form a linear hierarchy of Tukey immediate successors above a Ramsey ultrafilter, analogously to the $\mathcal{U}_{n}$ in this paper.

Finally, what happens at $\omega_{1}$ ? The straighforward limit of the spaces $\mathcal{R}_{\alpha}, \alpha<\omega_{1}$, does not produce a topological Ramsey space, as the auxiliary structure $\mathbb{S}_{\omega_{1}}$ would be uncountable and whatever countable tree $\mathbb{T}_{\omega_{1}}$ we were to use would not be able to code the structure of $\mathbb{S}_{\omega_{1}}$. In [10], Laflamme constructed a partial ordering $\mathbb{P}_{\aleph_{1}}$ which is a sort of amalgamation of all the $\mathbb{P}_{\alpha}, \alpha<\omega_{1}$. However, the ultrafilter $\mathcal{U}_{\aleph_{1}}$ forced by $\mathbb{P}_{\aleph_{1}}$ is a rapid p-point with no Ramsey ultrafilter Rudin-Keisler reducible to it.

\section{REFERENCES}

[1] Andreas Blass, The Rudin-Keisler ordering of P-points, Trans. Amer. Math. Soc. 179 (1973), 145-166. MR0354350 (50 \#6830)

[2] Andreas Blass, Ultrafilter mappings and their Dedekind cuts, Trans. Amer. Math. Soc. 188 (1974), 327-340. MR0351822 (50 \#4310)

[3] Natasha Dobrinen, High dimensional Ellentuck spaces and initial chains in the Tukey structure of non-p-points, 30 pp., submitted.

[4] Natasha Dobrinen and Stevo Todorcevic, Tukey types of ultrafilters, Illinois J. Math. 55 (2011), no. 3, 907-951 (2013). MR3069290

[5] Natasha Dobrinen and Stevo Todorcevic, A new class of Ramsey-classification theorems and their application in the Tukey theory of ultrafilters, Part 1, Trans. Amer. Math. Soc. 366 (2014), no. 3, 1659-1684, DOI 10.1090/S0002-9947-2013-05844-8. MR3145746

[6] Natasha Dobrinen, José G. Mijares, and Timothy Trujillo, Topological Ramsey spaces from Fraïssé classes, Ramsey-classification theorems, and initial structures in the Tukey types of p-points, 34 pp., submitted.

[7] Erik Ellentuck, A new proof that analytic sets are Ramsey, J. Symbolic Logic 39 (1974), 163-165. MR.0349393 (50 \#1887)

[8] Paul Erdős and Richard Rado, A combinatorial theorem, J. London Math. Soc. 25 (1950), 249-255. MR0037886 (12,322f)

[9] Alekos Kechris, Vladimir Pestov, and Stevo Todorcevic, Fraïssé limits, Ramsey theory, and topological dynamics of automorphism groups, Geom. Funct. Anal. 15 (2005), no. 1, 106-189, DOI 10.1007/s00039-005-0503-1. MR2140630 (2007j:37013)

[10] Claude Laflamme, Forcing with filters and complete combinatorics, Ann. Pure Appl. Logic 42 (1989), no. 2, 125-163, DOI 10.1016/0168-0072(89)90052-3. MR996504 (90d:03104)

[11] José G. Mijares, A notion of selective ultrafilter corresponding to topological Ramsey spaces, MLQ Math. Log. Q. 53 (2007), no. 3, 255-267, DOI 10.1002/malq.200510045. MR2330595 (2008k:03089)

[12] José G. Mijares and Gabriel Padilla, A Ramsey space of infinite polyhedra and the infinite random polyhedron, submitted.

[13] Pavel Pudlák and Vojtěch Rödl, Partition theorems for systems of finite subsets of integers, Discrete Math. 39 (1982), no. 1, 67-73, DOI 10.1016/0012-365X(82)90041-3. MR677888 (84i:05018)

[14] Dilip Raghavan and Stevo Todorcevic, Cofinal types of ultrafilters, Ann. Pure Appl. Logic 163 (2012), no. 3, 185-199, DOI 10.1016/j.apal.2011.08.002. MR2871264

[15] Stevo Todorcevic, Introduction to Ramsey spaces, Annals of Mathematics Studies, vol. 174, Princeton University Press, Princeton, NJ, 2010. MR2603812 (2011i:05263)

Department of Mathematics, University of Denver, 2360 S. Gaylord Street, Denver, COLORADO 80208

E-mail address: natasha.dobrinen@du.edu

Department of Mathematics, University of Toronto, Toronto, Canada M5S 2E4 And - Institut de Mathematiques de Jussieu, CNRS - UMR 7056, 75205 Paris, France

E-mail address: stevo@math.toronto.edu

E-mail address: stevo.todorcevic@imj-prg.fr 